\title{
User's and Reference Guide to the INEL RML/Analytical Radiochemistry Sample Tracking Database Version 1.00
}

D. A. Femec

Published September 1995

\section{Idaho National Engineering Laboratory Nuclear Engineering Department Lockheed Idaho Technologies Company Idaho Falls, Idaho 83415-7111}


,

$a$ 


\section{ABSTRACT}

This report discusses the sample tracking database in use at the Idaho National Engineering Laboratory (INEL) by the Radiation Measurements Laboratory (RML) and Analytical Radiochemistry. The database was designed in-house to meet the specific needs of the RML and Analytical Radiochemistry. The report consists of two parts, a user's guide and a reference guide. The user's guide presents some of the fundamentals needed by anyone who will be using the database via its user interface. The reference guide describes the design of both the database and the user interface. Briefly mentioned in the reference guide are the code-generating tools, CREATE_SCHEMA and BUILD_SCREEN, written to automatically generate code for the database and its user interface. The appendices contain the input files used by the these tools to create code for the sample tracking database. The output files generated by these tools are also included in the appendices.

\section{DISCLAIMER}

This report was prepared as an account of work sponsored by an agency of the United States Government. Neither the United States Government nor any agency thereof, nor any of their employees, makes any warranty, express or implied, or assumes any legal liability or responsibility for the accuracy, completeness, or usefulness of any information, apparatus, product, or process disclosed, or represents that its use would not infringe privately owned rights. Reference herein to any specific commercial product, process, or service by trade name, trademark, manufacturer, or otherwise does not necessarily constitute or imply its endorsement, recommendation, or favoring by the United States Government or any agency thereof. The views and opinions of authors expressed herein do not necessarily state or reflect those of the United States Government or any agency thereof. 


\section{DISCLAIMER}

Portions of this document may be illegible in electronic image products. Images are produced from the best available original document. 


\section{CONTENTS}

1. INTRODUCTION $\ldots \ldots \ldots \ldots \ldots \ldots \ldots \ldots \ldots \ldots \ldots \ldots \ldots \ldots \ldots \ldots \ldots \ldots \ldots$

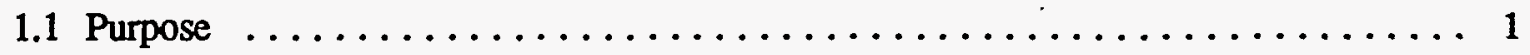

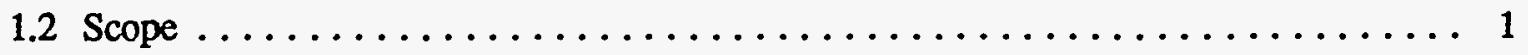

1.3 Future Major Revisions $\ldots \ldots \ldots \ldots \ldots \ldots \ldots \ldots \ldots \ldots \ldots \ldots \ldots$

1.4 Definitions, Acronyms, and Abbreviations $\ldots \ldots \ldots \ldots \ldots \ldots \ldots \ldots \ldots \ldots$

1.5 Registered Trademarks and Copyrights $\ldots \ldots \ldots \ldots \ldots \ldots \ldots \ldots \ldots \ldots$

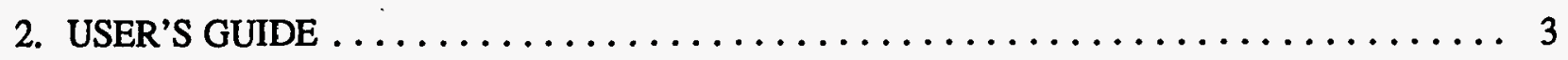

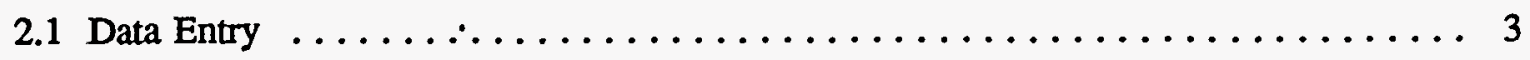

2.1.1 Use of Uppercase Letters $\ldots \ldots \ldots \ldots \ldots \ldots \ldots \ldots \ldots \ldots \ldots$

2.1.2 Striking The Return Key $\ldots \ldots \ldots \ldots \ldots \ldots \ldots \ldots \ldots \ldots \ldots$

2.1.3 Other Keys Of Use During Data Entry $\ldots \ldots \ldots \ldots \ldots \ldots \ldots \ldots$

2.2 Screen Legend $\ldots \ldots \ldots \ldots \ldots \ldots \ldots \ldots \ldots \ldots \ldots \ldots \ldots \ldots$

2.3 User Interface Options $\ldots \ldots \ldots \ldots \ldots \ldots \ldots \ldots \ldots \ldots \ldots \ldots \ldots$

2.3.1 Add a Sample Entry $\ldots \ldots \ldots \ldots \ldots \ldots \ldots \ldots \ldots \ldots \ldots \ldots$

2.3.1.1 Special Instructions $\ldots \ldots \ldots \ldots \ldots \ldots \ldots \ldots \ldots \ldots \ldots$

2.3.1.2 Possessor Tracking $\ldots \ldots \ldots \ldots \ldots \ldots \ldots \ldots \ldots \ldots$

2.3.1.3 Analyses Request Screens $\ldots \ldots \ldots \ldots \ldots \ldots \ldots \ldots \ldots$

2.3.1.3.1 Alpha Analyses $\ldots \ldots \ldots \ldots \ldots \ldots \ldots \ldots .10$

2.3.1.3.2 Special Instructions $\ldots \ldots \ldots \ldots \ldots \ldots \ldots \ldots$

2.3.1.3.3 Gross Alpha-Beta Analyses .............. 11

2.3.1.3.4 Beta Analyses $\ldots \ldots \ldots \ldots \ldots \ldots \ldots \ldots \ldots 11$

2.3.1.3.5 Gamma Analyses $\ldots \ldots \ldots \ldots \ldots \ldots \ldots \ldots 13$

2.3.1.4 Execution Sequence Tracking $\ldots \ldots \ldots \ldots \ldots \ldots \ldots \ldots$ 
2.3.2 Update a Sample Entry $\ldots \ldots \ldots \ldots \ldots \ldots \ldots \ldots \ldots \ldots \ldots .14$

2.3.3 Search for a Sample Entry (User-Specified Searches) $\ldots \ldots \ldots \ldots \ldots 16$

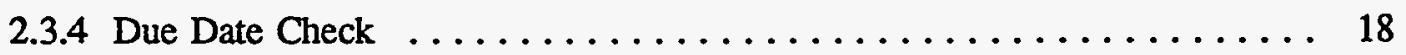

2.3.5 List Last Search Results . . . . . . . . . . . . . . . . . . 19

2.3.6 Exit from this Database Session .................... 19

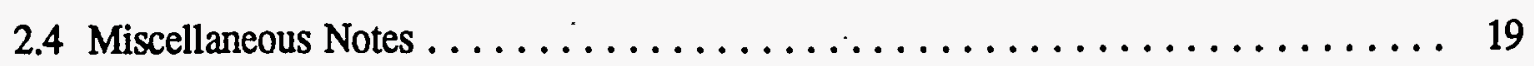

2.4.1 "When the nightmare comes to pass ..." $\ldots \ldots \ldots \ldots \ldots \ldots \ldots$

2.4.1.1 System Crashes ........................ 19

2.4.1.2 Database-Related Errors $\ldots \ldots \ldots \ldots \ldots \ldots \ldots \ldots \ldots .20$

2.4.2 Bar Code Labels .......................... 20

2.4.2.1 Affixing Bar Code Labels to Sample Containers ........ 20

2.4.2.2 Replacing Printer Ribbons and Label Rolls .......... 21

2.4.2.3 What if the bar code printer stops printing labels? ....... 22

2.4.3 What to do with Suggestions or Comments on the Database or the User

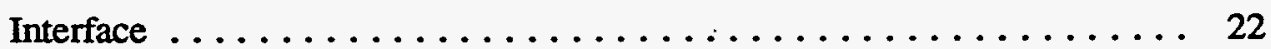

3. DESIGN OF THE DATABASE $\ldots \ldots \ldots \ldots \ldots \ldots \ldots \ldots \ldots \ldots \ldots \ldots \ldots \ldots \ldots \ldots \ldots$

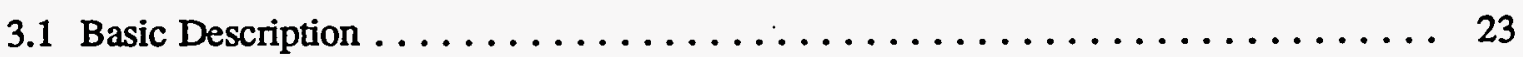

3.1.1 Tables and their Fields and Indexes for Sorting $\ldots \ldots \ldots \ldots \ldots \ldots 23$

3.1.2 Domains and Attributes $\ldots \ldots \ldots \ldots \ldots \ldots \ldots \ldots \ldots \ldots \ldots \ldots \ldots$

3.1.3 Storage Areas and Snapshot Files $\ldots \ldots \ldots \ldots \ldots \ldots \ldots \ldots \ldots \ldots$

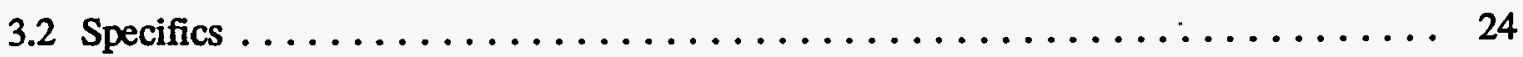

3.2.1 Names of Tables, Storage Areas, and Snapshot Files ........... 24

3.2.2 Names and Descriptions of Domains $\ldots \ldots \ldots \ldots \ldots \ldots \ldots \ldots \ldots 24$ 。

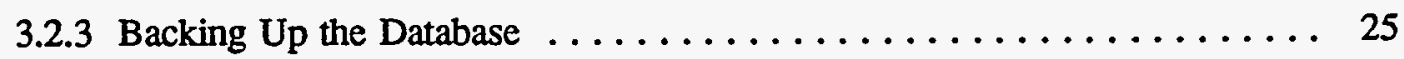


4. DESIGN OF THE USER INTERFACE $\ldots \ldots \ldots \ldots \ldots \ldots \ldots \ldots \ldots \ldots \ldots \ldots \ldots$

4.1 Basic Description . . . . . . . . . . . . . . . . . . . 27

4.2 Specifics $\ldots \ldots \ldots \ldots \ldots \ldots \ldots \ldots \ldots \ldots \ldots \ldots \ldots \ldots \ldots \ldots \ldots \ldots$

4.2.1 Data Storage Variables and Arrays . . . . . . . . . . . . 27

4.2.1.1 Storage of Data $\ldots \ldots \ldots \ldots \ldots \ldots \ldots \ldots \ldots \ldots$

4.2.1.2 Conversion of Character Data to Numerical Data . . . . . . . 28

4.2.1.3 Error Checking $\ldots \ldots \ldots \ldots \ldots \ldots \ldots \ldots \ldots \ldots \ldots$

4.2.1.3.1 "a" for Anything $\ldots \ldots \ldots \ldots \ldots \ldots \ldots \ldots \ldots$

4.2.1.3.2 "d" for Date $\ldots \ldots \ldots \ldots \ldots \ldots \ldots \ldots \ldots \ldots$

4.2.1.3.3 "l" for Length $\ldots \ldots \ldots \ldots \ldots \ldots \ldots \ldots \ldots \ldots$

4.2.1.3.4 " $\mathrm{t}$ " for Time $\ldots \ldots \ldots \ldots \ldots \ldots \ldots \ldots \ldots$

4.2.1.3.5 "u" for Units of Time $\ldots \ldots \ldots \ldots \ldots \ldots \ldots \ldots$

4.2.1.3.6"?" for Questions $\ldots \ldots \ldots \ldots \ldots \ldots \ldots \ldots \ldots \ldots$

4.2.2 Communication between the User Interface and the Database . . . . . . 30

4.2.2.1 Forms of SQL Used $\ldots \ldots \ldots \ldots \ldots \ldots \ldots \ldots \ldots \ldots$

4.2.2.2 Commencing a Database Transaction ............ 30

4.2.2.3 Add a Sample Entry . . . . . . . . . . . . . 31

4.2.2.4 Search for a Sample Entry . . . . . . . . . . . . 32

4.2.2.5 Due Date Checks . . . . . . . . . . . . . . 33

4.2.2.6 Update a Sample Entry . . . . . . . . . . . . 34

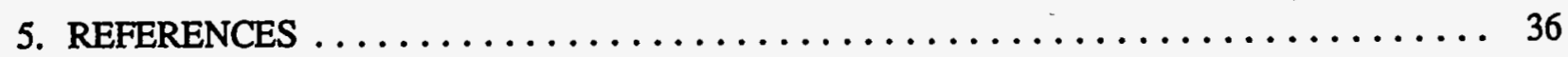




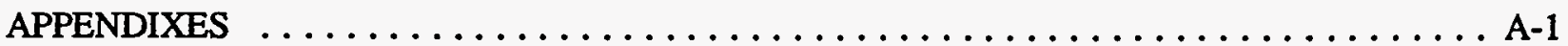

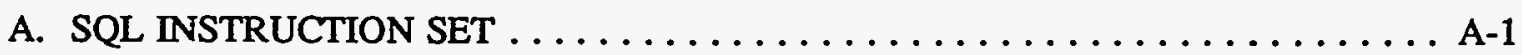

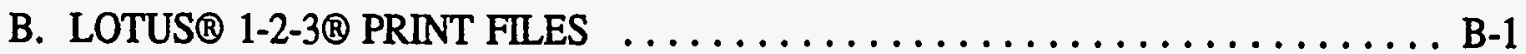

B.1 Entity-Sorted List $\ldots \ldots \ldots \ldots \ldots \ldots \ldots \ldots \ldots \ldots \ldots \ldots \ldots \ldots \ldots \ldots \ldots \ldots$

B.2 Screen-Sorted List $\ldots \ldots \ldots \ldots \ldots \ldots \ldots \ldots \ldots \ldots \ldots \ldots \ldots \ldots \ldots \ldots$

C. EXECUTION OF CREATE_SCHEMA $\ldots \ldots \ldots \ldots \ldots \ldots \ldots \ldots \ldots \ldots \ldots \ldots \ldots \ldots$

D. DATABASE BACKUP $\ldots \ldots \ldots \ldots \ldots \ldots \ldots \ldots \ldots \ldots \ldots \ldots \ldots \ldots \ldots \ldots \ldots \ldots \ldots$

E. ARRAY AND SCALAR DECLARATIONS $\ldots \ldots \ldots \ldots \ldots \ldots \ldots \ldots \ldots$ E-1

F. SCREEN TEMPLATES SUBMTTED TO BUILD_SCREEN $\ldots \ldots \ldots \ldots \ldots \ldots$ F-1

F.1 Screen Template for the Main (M) Screen ................ F-1

F.2 Screen Template for the Possessors $\ldots \ldots \ldots \ldots \ldots \ldots \ldots \ldots \ldots$ F-1

F.3 Screen Template for the Alpha Analyses $\ldots \ldots \ldots \ldots \ldots \ldots \ldots \ldots$ F-2

F.4 Screen Template for the Beta Analyses $\ldots \ldots \ldots \ldots \ldots \ldots \ldots \ldots \ldots$ F-2

F.5 Screen Template for the Gross Alpha-Beta Analyses $\ldots \ldots \ldots \ldots \ldots \ldots$ F-3

F.6 Screen Template for Gamma Analyses $\ldots \ldots \ldots \ldots \ldots \ldots \ldots \ldots$ F-3

F.7 Screen Template for Special Instructions $\ldots \ldots \ldots \ldots \ldots \ldots \ldots \ldots \ldots$ F-4

F.8 Screen Template for RML/Analytical Radiochemistry Analysts ........ F-4

\section{FIGURES}

Figure 1. Mapping of Arrow and Keypad Keys and of Some Special Function Keys. . . . . . . 4

Figure 2. Options Request Screen of the Sample Tracking Database User Interface. ........ 5

Figure 3. A Fresh Main Screen for Adding a Sample Entry. . . . . . . . . . . . . 7

Figure 4. A Fresh Special Instructions Screen. .................... 8

Figure 5. A Fresh Possessor Tracking Screen. $\ldots \ldots \ldots \ldots \ldots \ldots \ldots \ldots \ldots \ldots$

Figure 6. A Possessor Tracking Screen with the Possessor List Overlaid. . . . . . . . . . . . . 9 
Figure 7. The Alpha Analyses Request Screen. $\ldots \ldots \ldots \ldots \ldots \ldots \ldots \ldots \ldots \ldots \ldots$

Figure 8. A Special Instructions Screen Following an Alpha Analyses Request Screen. . . . . . 11

Figure 9. The Gross Alpha-Beta Analyses Request Screen. . . . . . . . . . . . . . 12

Figure 10. The Beta Analyses Request Screen. .................... 12

Figure 11. The Gamma Analyses Request Screen. $\ldots \ldots \ldots \ldots \ldots \ldots \ldots \ldots$

Figure 12. Options Request Screen After a Successful Search. . . . . . . . . . . . . 15

Figure 13. Options Request Screen During Initiation of the Update Option. . . . . . . . 16

Figure 14. A Main Screen for Updating a Sample Entry. . . . . . . . . . . . . . 17

Figure 15. A Fresh Main Screen for Searching for a Sample Entry. . . . . . . . . . 18

Figure 16. How to Affix Bar Code Labels to Cylindrical Sample Containers. . . . . . . . . . 21

\section{TABLES}

Table 1. Arrays and Scalars Used in Coding the User Interface. . . . . . . . . . . . . 29 


\section{INTRODUCTION}

\subsection{Purpose}

The need for secure and auditable tracking of samples submitted to the INEL Radiation Measurements Laboratory (RML) and Analytical Radiochemistry for analysis is clear. This need has led to the development of the database and user interface described herein.

\subsection{Scope}

Version 1.00 of the database represents the essential capabilities necessary for such a database:

- The sample coordinator can track the whereabouts of samples within the RML and Analytical Radiochemistry.

- The sample coordinator can monitor the progress of requested analyses.

- Submitters can provide information on how the requested analyses are to be performed.

- Analysts and operators can obtain basic descriptive information on a sample and the types of analyses to be performed on it.

- Analysts and operators can document the completion of their analyses and the promulgation of the results.

\subsection{Future Major Revisions}

Major revisions could provide the following additional capabilities:

- The sample coordinator will be able to generate printed versions of any of the screens, including expanded listings such as all possessors of a given sample.

- Submitters will be able to provide more detailed information on how the requested analyses are to be.performed.

- Analysts and operators will be able to retrieve and enter pertinent analysis parameters.

- Certain programs used in preparing VAXGAP gamma-ray spectral data files, such as HEAD (see Reference 1), will talk directly with the database.

- RML operators will be able to submit gamma-ray spectra to the appropriate analysis program directly from a user interface.

- Operator-approved results from these analysis programs will be stored in this or a related database.

- Analyst-approved batch analysis results from gamma-ray spectra will be stored in this or a related database. 
- Other analysts will be able to enter their analysis results.

- RML Data Analysis and Management QA/QC programs will retrieve results from this or a related database.

These modifications will extend the security and audit safety nets inherent in a database over the entire process of sample-received to analysis-performed to sample-returned-and-results-reported. Other modifications will be implemented as the needs arise and are identified.

\subsection{Definitions, Acronyms, and Abbreviations}

Terminology related to Digital Equipment Corporation's VMS operating system, DECnet communication protocol, DECwindows Motif graphical user interface, FORTRAN compiler, GKS (Graphical Kemel System) plotting routines, Rdb relational database software, and SQL precompiler, is desirable. Otherwise, this document explicitly defines technical terms as they are pre thed and avoids unnecessary technical jargon for the sake of the general reader.

\subsection{Registered Trademarks and Copyrights}

DEC, DECnet, DECwindows, GKS (Graphical Kemel System), Rdb, VAX, and VMS are registered trademarks of Digital Equipment Corporation (DEC). Motif is a registered trademark of Open Systems Foundation. INTERMEC is a registered trademark of INTERMEC Corporation. Lotus and 1-2-3 are registered trademarks of Lotus Corporation. 


\section{USER'S GUIDE}

\subsection{Data Entry}

The following subsections describe how data is accepted by the user interface before being entered into the database. Some special function keys for simplifying data entry are also presented.

\subsubsection{Use Of Uppercase Letters}

The user interface converts to uppercase all letters of the alphabet entered by the user. (A query could be a data field to be filled, or a request or question to be answered.) Searches of the database are case-sensitive; this means that searches distinguish between " $\mathrm{a}$ " and " $\mathrm{A}$," for example. Forcing all letters to uppercase eliminates the need for a user to remember how a name was entered; for example, "Was that 'Jones' or 'JONES' I entered?"

\subsubsection{Striking The Return Key}

Striking the return key is the ubiquitous key stroke for terminating an interactive entry of data into a requesting program. Within the user interface, the effect of striking the retum key depends on the application. Consider the options request screen, presented upon entering the user interface. After the user chooses an option and enters the corresponding highlighted letter, striking the return key begins execution of that option. Within a data entry screen, striking the return key can move the cursor to the next accessible data field. (An accessible data field is one into which data can be entered; that is, the field is not fixed. See Screen Legend, section 2.2.) During a listing of sample entries retrieved from the database, striking the return key tells the user interface to present the next screen of entries. All of these instances will be demonstrated in more detail in subsequent sections.

\subsubsection{Other Keys Of Use During Data Entry}

Within a data entry screen, there are other keys that can be used to move the cursor from field to field. These include the arrow keys and some keypad and special function keys. For example, when it is time to move to the next screen during data entry, strike the "Do" special function key (or control-Z). Or, to print an additional bar code label for the sample entry currently displayed, strike the F17 special function key. Figure 1 displays the functional mapping of the arrow and keypad keys and of the special function keys located above them. This figure can be obtained during data entry by striking the "Help" special function key (or keypad key PF2).

The arrow and keypad key mappings are similar to those mappings used in most VMS editors. Note, though, that all of the delete keys (keypad comma for "delete character," keypad dash for "delete word," . . . ) erase the entire contents of the current data field; there is no editing-of-an-entry capability. Also, all of the "move-to-the-next" keys (keypad 1 for "next word," keypad 3 for "next character," ... ) move the cursor to the next accessible data field. "Prev Screen" and "Next Screen" move the cursor to the top-most and bottom-most accessible data field of the current screen, respectively.

During cursor movement, any data entry screen acts like a sphere. Suppose the cursor is in the last accessible field of a screen and the user strikes the return key. The cursor can move to the first accessible field of that screen. Or suppose the cursor is on the top-most accessible row of a screen and the user strikes the up-arrow key. The cursor can move to the bottom-most accessible row of that screen. 


\begin{tabular}{|l|l|}
\hline Help & $\begin{array}{l}\text { Next } \\
\text { Screen }\end{array}$ \\
\hline
\end{tabular}

\begin{tabular}{|l|l|l|l|}
\hline $\begin{array}{l}\text { Print } \\
\text { Bar code }\end{array}$ & & & \\
\hline
\end{tabular}

\begin{tabular}{|l|l|l|}
\hline & $\begin{array}{l}\text { Ditto } \\
\text { Entry }\end{array}$ & $\begin{array}{l}\text { Delete } \\
\text { Entry }\end{array}$ \\
\hline & $\begin{array}{l}\text { Top of } \\
\text { Screen }\end{array}$ & $\begin{array}{l}\text { End of } \\
\text { Screen }\end{array}$ \\
\hline & $\begin{array}{l}\text { Move } \\
\text { Up }\end{array}$ & \\
\hline $\begin{array}{l}\text { Move } \\
\text { Left }\end{array}$ & $\begin{array}{l}\text { Move } \\
\text { Down }\end{array}$ & $\begin{array}{l}\text { Move } \\
\text { Right }\end{array}$ \\
\hline
\end{tabular}

\begin{tabular}{|l|l|l|l|}
\hline $\begin{array}{l}\text { Abort } \\
\text { Program }\end{array}$ & Help & & $\begin{array}{l}\text { Delete } \\
\text { Entry }\end{array}$ \\
\hline $\begin{array}{l}\text { Top/End } \\
\text { Screen }\end{array}$ & & $\begin{array}{l}\text { Delete } \\
\text { Entry }\end{array}$ \\
\hline Advance & Backup & $\begin{array}{l}\text { Delete } \\
\text { Entry }\end{array}$ & $\begin{array}{l}\text { Delete } \\
\text { Entr }\end{array}$ \\
\hline $\begin{array}{l}\text { Next } \\
\text { Entry }\end{array}$ & $\begin{array}{l}\text { End of } \\
\text { Line }\end{array}$ & $\begin{array}{l}\text { Next } \\
\text { Entry }\end{array}$ & $\begin{array}{l}\text { Nex } \\
\text { Entr }\end{array}$ \\
\cline { 1 - 2 } $\begin{array}{l}\text { Next } \\
\text { Line }\end{array}$ & & & \\
\hline
\end{tabular}

Figure 1. Mapping of Arrow and Keypad Keys and of Some Special Function Keys.

\subsection{Screen Legend}

The cursor, on most monitors, will appear as a blinking, upright rectangle ( $\square$ ). The cursor tells the user where the next key stroke will be read.

The rendition (or appearance) of a field depends on whether an entry into that data field is optional (regular text) or required (bold text). Fixed fields cannot be changed (reverse video text; presented here as double-underlined). This will be demonstrated in the figures of subsequent subsections. An illegal entry causes the field's regular or bold text to blink (that is, alternate between normal and reverse video) while the monitor beeps.

\subsection{User Interface Options}

The user starts the user interface by typing STDB (for sample tracking database) at the DCL prompt and then striking the return key. Figure 2 displays the options request screen presented to the user upon entering the user interface. (This screen also appears upon completion of any of the options, except for exiting from the database session.)

The user interface presents six cho $35:$

- Add a Sample Entry 


\section{RML/Radiochemistry Sample Tracking Database}

\section{Add a Sample Entry}

Update a Sample Entry

Search for a sample Entry

Due Date Check

\section{List Last Search Results}

Exit from this Database session

\section{Enter your choice $(A, U, S, D, L, E)$ :}

Figure 2. Options Request Screen of the Sample Tracking Database User Interface.

- Update a Sample Entry

- Search for a Sample Entry

- Due Date Check

- List Last Search Results and

- Exit from this Database Session.

The following subsections describe these options.

\subsubsection{Add a Sample Entry}

The tracking of samples begins with their entry into the database. The sample coordinator should make only one entry for each sample and should enter samples as they arrive. Prior to adding a sample, the following information needs to be at hand: 
- who is submitting the sample for analysis

- is there a chain-of-custody form associated with the sample

- what identifier (name or identification code?) does the submitter use to distinguish this sample from any other

- in what form is the sample (soil, water, ... )

- how large is the sample

- does the sample contain hazardous chemicals

- has the sample been surveyed

- what analyses are to be performed

- how are the analyses to be performed

- who should be contacted in case there are any technical questions regarding the sample or the analyses to be performed

- when are the results due

- to whom are the results to be reported

- what special requirements does the submitter have for the analyses and handling of the sample

- to whom is the sample to be retumed and

- which charge number is to be used to pay for the analyses.

The user interface automatically generates a unique sample tracking identification code for each sample entry added to the database. The identification code is twelve digits long, constructed by concatenation of the six-digit integer representations of the current date (mmddyy) and time (hhmmss). Upon successful addition of an entry to the database, the bar code printer prints labels containing the identification code. The code appears literally and in the CODE 39 symbology on the labels. This way the code can be read even if a functioning bar code scanner is not available.

This unique identification code, and the date the sample was entered in the database, cannot be changed by the sample coordinator. The user interface supplies both. These two fields will appear in reverse video on the monitor screen (and double-underlined here). Figure 3 is a depiction of a fresh main screen for adding a sample entry. Note that the cursor is in the data field "Completed" and not in the data field "Tracking ID." Since the "Tracking ID" and "Date Entered" fields cannot be changed, the cursor cannot be moved into them.

Some of the fields in the figure appear in bold text ("Desired Completion Date," "Sample Name," ... ). Recall that these fields must be filled before the user interface will go to the next screen.

The user interface assigns the sample coordinator as the current possessor, the sample coordinator 


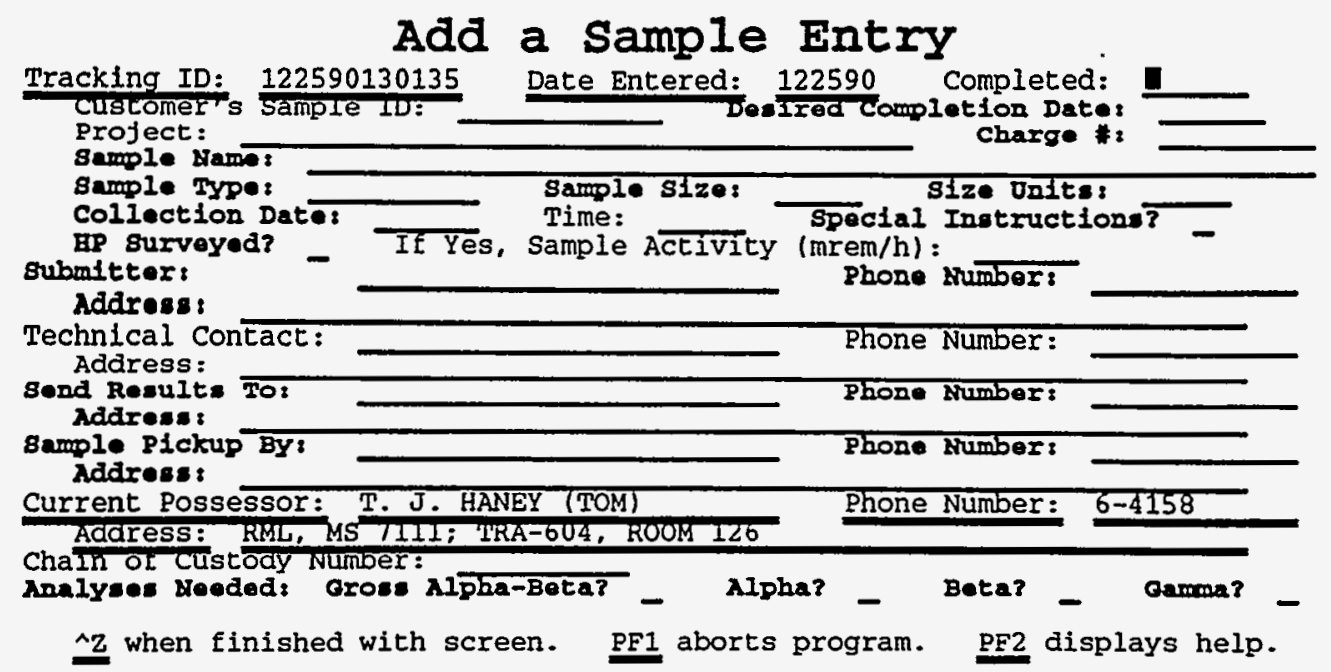

Figure 3. A Fresh Main Screen for Adding a Sample Entry.

is the first possessor of any sample received for analysis. The current possessor cannot be updated in the main screen; it can be updated in the possessor tracking screen, as described in Possessor Tracking, section 2.3.1.2.

The main data screen presents a prime situation to use the "ditto" key. For example, "Customer's Sample ID" dittos to "Sample Name." With the cursor in the "Sample Name" field, depressing the "ditto" key (see Figure 1) copies the contents of the "Customer's Sample ID" field to the "Sample Name" field. Also, the set of "Submitter" fields ditto to the "Technical Contact" fields. These in tum ditto to the "Send Results To" fields, which ditto to the "Sample Pickup By" fields.

If, after adding one sample, the sample coordinator asks to add another, the screens displayed will contain most of the same information entered for the prior sample. In this way the user interface simplifies the entry of a series of related samples. The sample tracking identification code will be different and the date entered may be different. The rest of the information can be changed or left unaltered. What appears on the screens will go into the database.

2.3.1.1 Special Instructions. If the user enters a "Y" into the "Special Instructions?" field, the interface presents the special instructions screen (see Figure 4). The special instructions screen can be thought of as a sub-screen of the main screen. The information contained in it is part of the main screen. The special instruction screen also serves as sub-screens for the specific analysis screens, as will be demonstrated later (Special Instructions, section 2.3.1.3.2).

At the top of the special instructions screen are two fixed fields, "Tracking ID" and "Sample Name." These two fields will appear at the top of all other screens needed for this sample entry. This serves to remind the sample coordinator which sample is being entered.

At this point, the user interface fills all of the "From" fields in the special instruction screen with "Main Screen." The user interface assigns the contents of the available "From" fields based on which 


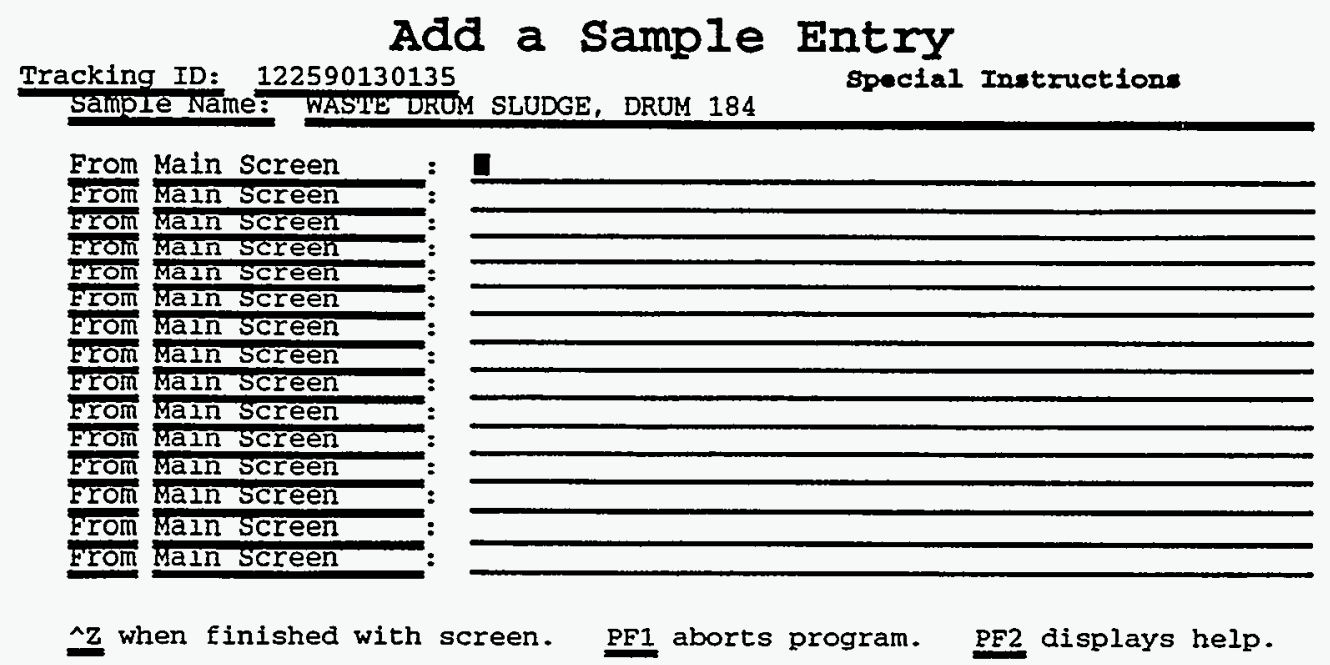

Figure 4. A Fresh Special Instructions Screen.

screen calls for the special instructions screen. The interface also displays all previously entered special instructions; this will be demonstrated later in Special Instructions, section 2.3.1.3.2. The sample coordinator fills the instruction fields as desired. Blank lines between instructions should be avoided. Also, since duplicate lines are undesirable, there are no "ditto" links in the special instructions screen.

2.3.1.2 Possessor Tracking. After the main screen (and the special instructions screen, if needed), the user interface presents the possessor tracking screen. The sample coordinator uses this screen to update the current possessor of the sample. (Please note that this is not a chain of custody. A chain of custody is not required to be maintained within a laboratory performing analyses. The chain of custody must include the sample's arriving at and departing from the laboratory, though.) The user interface presents the current possessor and blanks for two additional possessors (see Figure 5). For samples just received for analyses, the sample coordinator is the first possessor.

The possessors are numbered sequentially. Note that none of the current possessor fields can be changed, except the "Date sample relinquished" field. After the sample coordinator fills this field, the cursor moves to the next set of possessor fields. Since this set of fields is blank, a new possessor needs to be entered. The user interface presents a list of possible possessors (all the analysts/operators within the RML and Analytical Radiochemistry) for the sample coordinator to choose from. This list appears as an overlay to the possessor tracking screen (see Figure 6).

This overlaid list screen is simpler than other data entry screens. The only fields in which entries can be made are the underlined blanks, one following each name. The sample coordinator chooses a name in the list by typing any character in the appropriate blank. If the sample coordinator puts characters in more than one blank, the interface recognizes only the first one. Cursor movement between fields and deletion of entries made in fields operate the same way as with a data entry screen.

If the sample coordinator chooses a listed possessor, the user interface automatically fills the appropriate fields. The interface presents a best guess in the "Reason for possession" field; the sample 


\section{Add a Sample Entry}

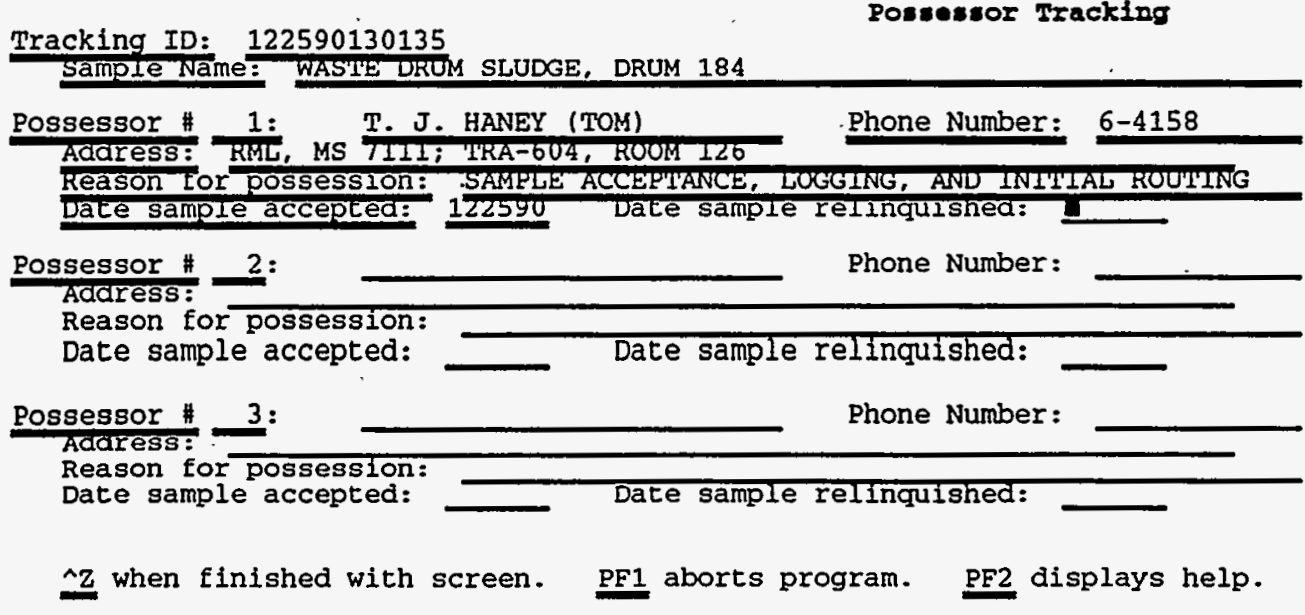

Figure 5. A Fresh Possessor Tracking Screen.

\section{Add a Sample Entry}

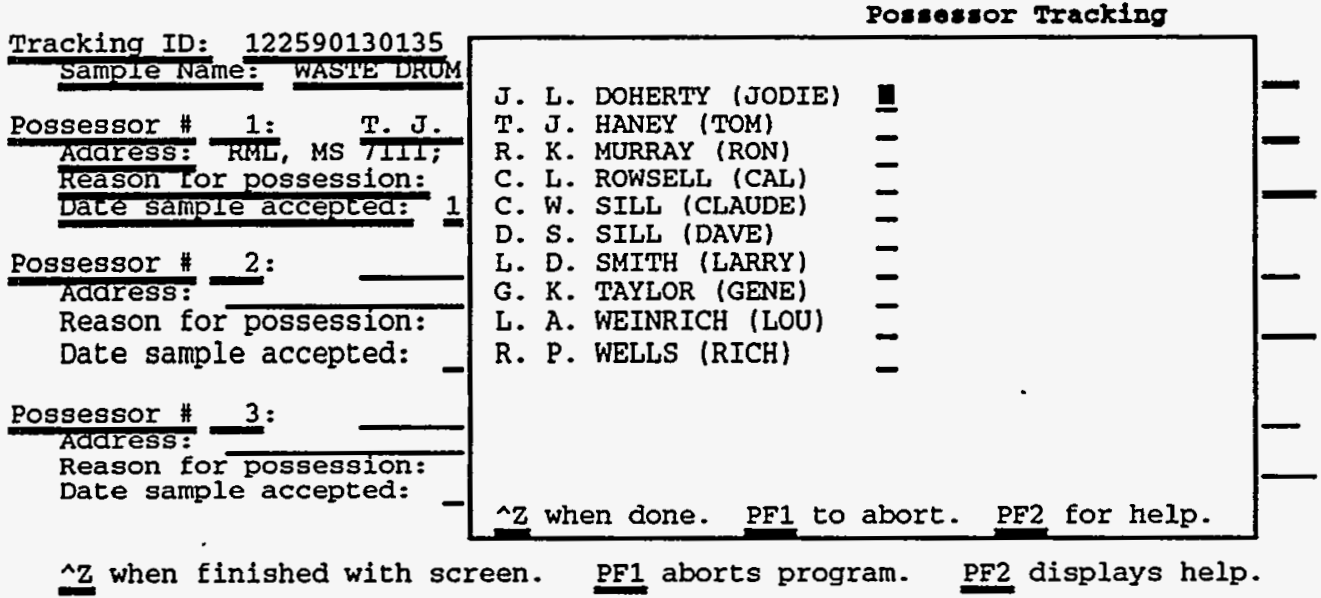

Figure 6. A Possessor Tracking Screen with the Possessor List Overlaid.

coordinator made need to change this. The sample coordinator does not need to choose an item from the list before finishing with the list screen and can instead fill the possessor fields manually.

As already noted, the interface presents two blanks for additional possessors. This allows the sample coordinator to accept the sample from the prior possessor and pass it on to someone else. Only the "Date sample relinquished" fields are "ditto" linked. 
$\because$ 3.1.3 Analyses Request Screens. The user interface then presents the desired analyses request : reens. Here the sample coordinator specifies which analyses are to be performed and any data acquisition requirements. Corresponding fields within an analyses request screen (for example, all "Results report citation" fields) are "ditto" linked.

2.3.1.3.1 Alpha Analyses - Figure 7 presents the alpha analyses request screen. The following alpha analyses are currently available:

- uranium isotopes

- thorium isotopes

- plutonium isotopes

- americium-241 (Am-241) separate from plutonium-238 (Pu-238)

- americium-241 combined with pl: tium-238

- total spectrometric alpha and

- other (i.e., special requests).

\section{Add a Sample Entry}

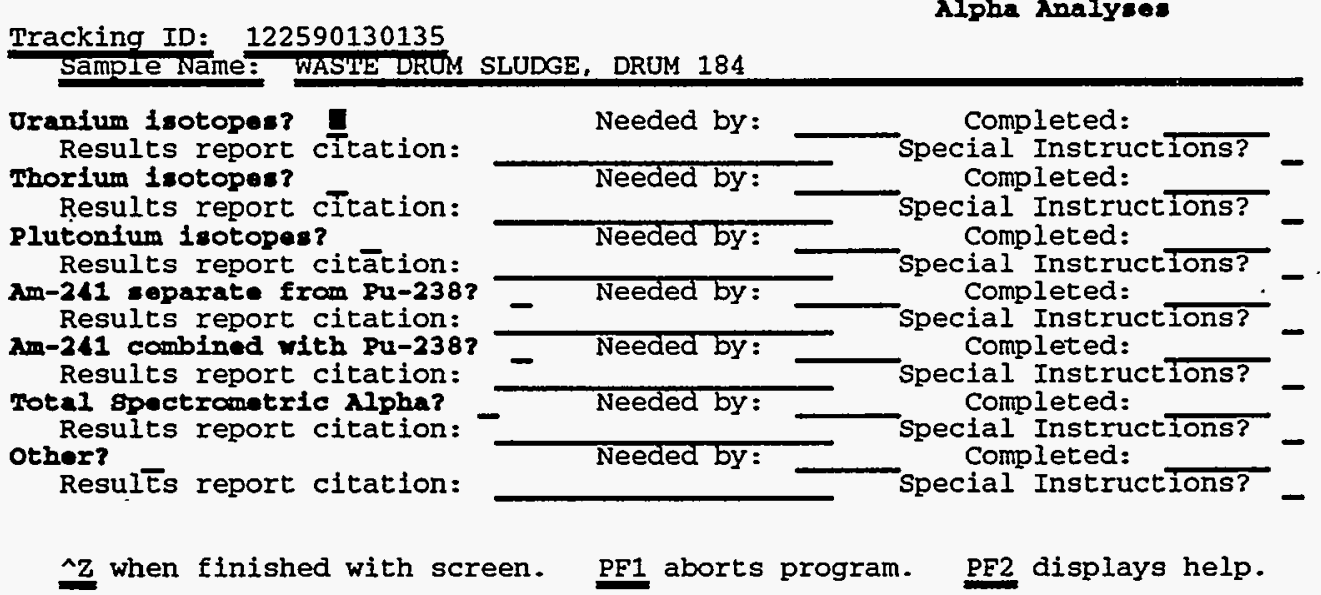

Figure 7. The Alpha Analyses Request Screen.

For these analyses the submitter can specify when tite results are needed and.any special instructions. The analyst can document the completion of an analy: ad cite the appropriate report.

2.3.1.3.2 Speclal Instructlon Iote that there is a "Special Instructi, ?" field associated with each type of analysis. Suppose th. 3 ismitter has some special instructions to go with a requested analysis. The user interface presents a special instructions screen (see Figure 8) after the sample 
coordinator finishes with the alpha analyses request screen. The special instructions entered from the main screen appear first. The interface places the cursor at the first data field for special instructions from the alpha analyses request screen. New special instructions can be entered and any instruction on the screen can be changed.

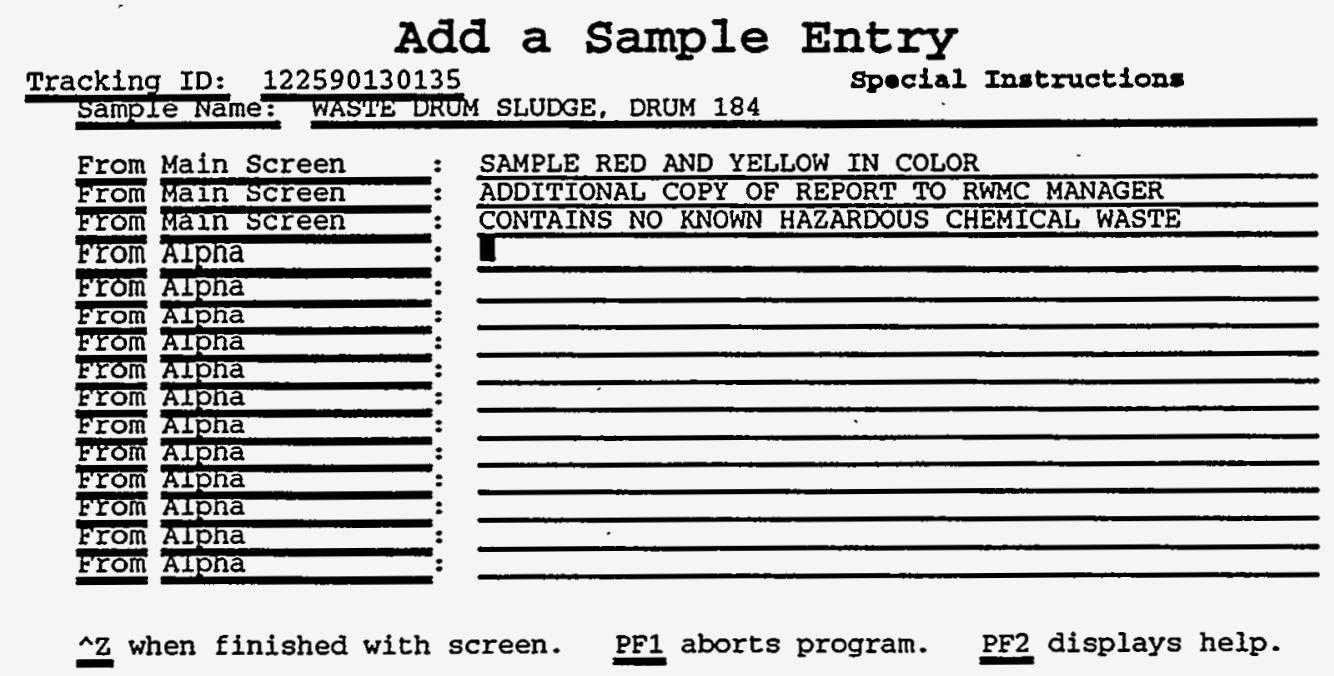

Flgure 8. A Special Instructions Screen Following an Alpha Analyses Request Screen.

The following figures, 9-11, contain the three remaining analyses request screens.

2.3.1.3.3 Gross Alpha-Beta Analyses - The following gross alpha-beta analyses are currently available:

- for air filters and

- other (i.e., special requests).

For these analyses the submitter can specify the duration of the count, when the results are needed, and any special instructions. The analyst can document the completion of an analysis and cite the appropriate report.

2.3.1.3.4 Beta Analyses - The following beta analyses are currently available:

- strontium-90 (Sr-90)

- strontium-89 and -90

- total strontium

- tritium (H-3) and 


\section{Add a Sample Entry}

Grose Alpha-Beta Analyees

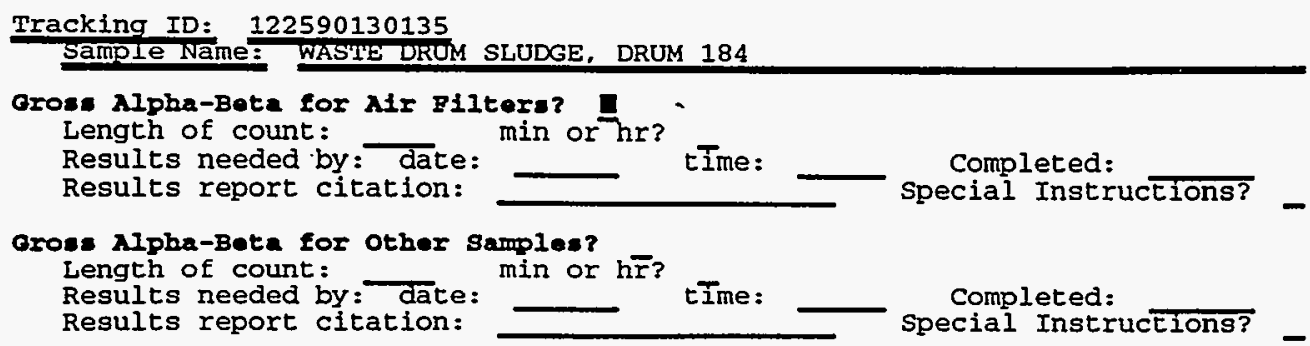

^ $\mathrm{Z}$ when finished with screen. PF1 aborts program. $\quad$ PF2 displays help.

Figure 9. The Gross Alpha-Beta Analyses Request Screen.

- other: nickel-63 (Ni-63), iron-55 (Fe-55), sulfur-35 (S-35), plutonium-241 (Pu-241), or any other special requests.

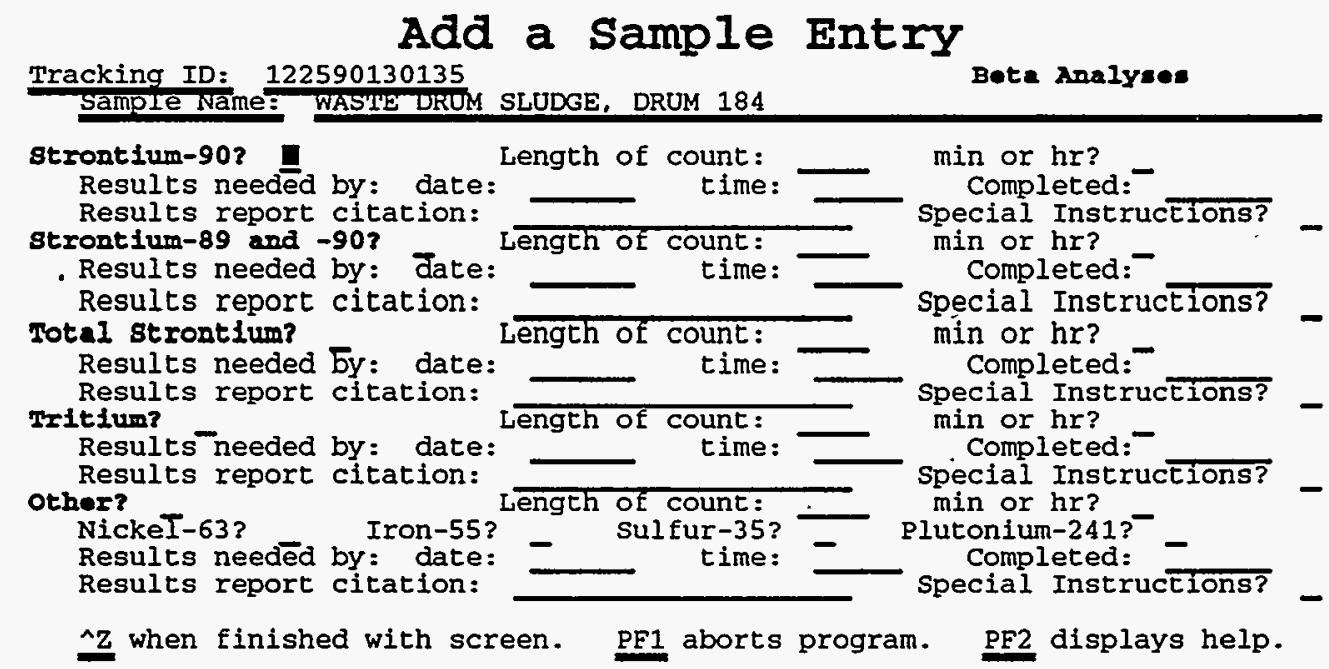

Figure 10. The Beta Analyses Request Screen.

For these analyses the submitter can specify the duration of the count, when the results are needed, and any special instructions. The analyst can document the completion of an analysis and cite the appropriate report. 
2.3.1.3.5 Gamma Analyses - The following gamma analyses are currently available:

- screening/shipping counts (for deciding whether a sample has an activity above two nanocuries per gram - above this level the sample must be classified as radioactive for shipping as per U.S. Department of Transportation orders)

- full isotopic and

- other (i.e., special requests).

\section{Add a Sample Entry}

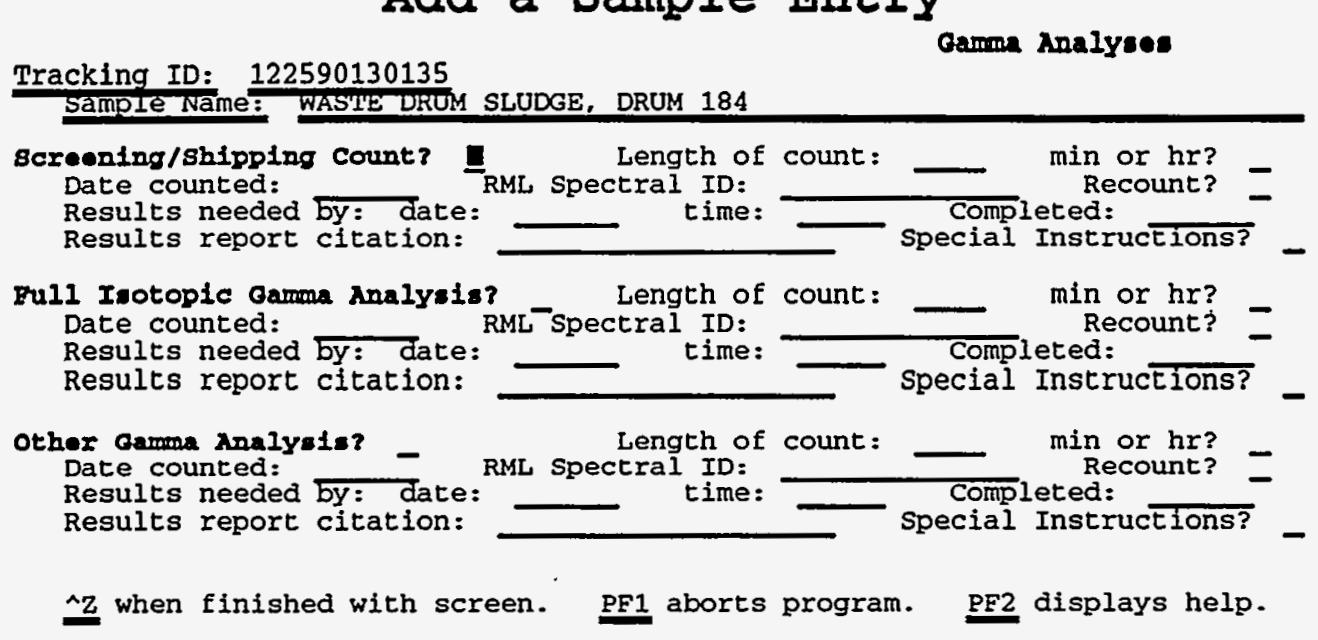

Figure 11. The Gamma Analyses Request Screen.

For these analyses the submitter can specify the duration of the count, when the results are needed, and any special instructions. The operator can record the spectrum acquisition date, the RML identification code for the spectrum, and whether the spectrum is from a recount. The analyst can document the completion of an analysis and cite the appropriate report.

2.3.1.4 Executlon Sequence Tracking. Once all of the appropriate data screens are properly filled in, the user interface inserts the new entry into the database. As this is occurring, the user interface clears the monitor and presents an execution sequence tracking display. A sequence of messages such as the following will appear in the display:

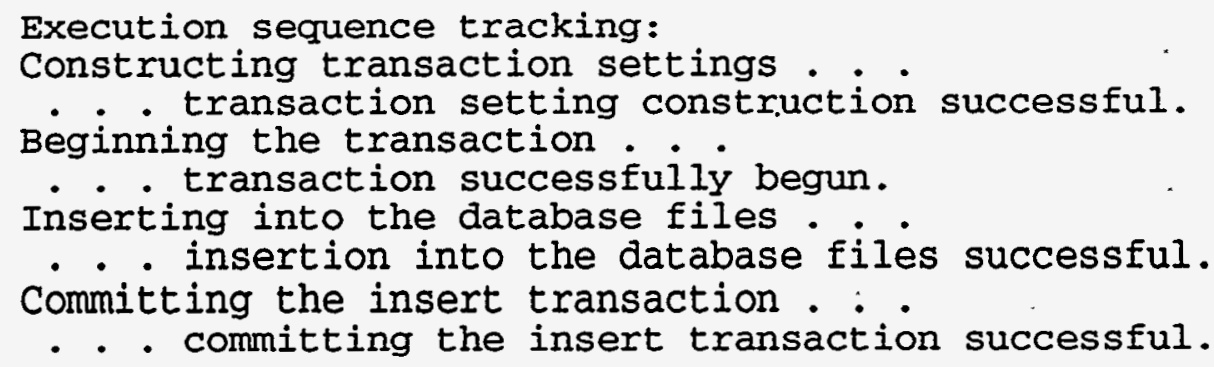


Any time the user interface accesses the database, it will keep the user informed as to the progress 0 i $c$ database transaction in just this way. Execution sequence tracking serves two functions. First, it let ie user know that the user interface is still working. Second, it provides the database programmer with suise additional information in case the user interface in some way fails while attempting to perform a transaction.

As soon as the database transaction is successfully completed, the execution sequence tracking display disappears and the options request screen reappears on the monitor.

\subsubsection{Update a Sample Entry}

The interface asks the sample coordinator to "... enter the number of the entry to update or the tracking id of the entry to be retrieved." After any successful search, the user interface displays the number of entries retrieved on the options request screen (see Figure 12). The first way in which the sample coordinator can specify the sample entry to be updated makes use of the list of retrieved sample; Here the sample coordinator enters the ordinal number of the desired entry in the list (see Figure 13). $T$. list is explained in more detail below.

The second way allows the sample coordinator to obtain directly the entry for a specific sample tracking identification code without first constructing a search for the entry. The sample coordinator simply enters the sample tracking identification code. (Remember that all sample tracking identification codes are twelve digits long and that any leading or trailing zeros must be included.) The user interface then performs a search for the entry.

Once the sample coordinator specifies the desired entry, the user interface attempts to retrieve it. First the interface searches for the entry. Upon finding the entry, the interface then retrieves it. Finally, the interface presents a screen akin to that presented in Figure 14. 


\title{
RML/Radiochemistry Sample Tracking Database
}

\section{A Ad a Sample Entry}

\section{Update a Sample Entry}

\author{
Search for a sample Entry
}

\section{Due Date Check}

\section{List Last Search Results}

\section{Exit from this Database session}

\section{Enter your choice $(A, U, S, D, L, E)$ :}

57 entries were found in the last search.

Figure 12. Options Request Screen After a Successful Search.

If the entry does not exist (suppose the sample coordinator has entered a bogus sample tracking identification code such as 123456879012), two things happen. First, the following appears at the end of the execution sequence tracking display:

$$
\begin{aligned}
& \text { Rolling back the transaction because of an error... } \\
& \text {. roll back successful. }
\end{aligned}
$$

Second, because the user interface recognizes that no entry was found, "No entry exists for this tracking id!" appears in big, blinking text at the bottom of the options request screen.

For updating a sample entry, the user interface behaves much as it does for adding a sample entry. The screens will have the same appearance. The screen heading will be "Update a Sample Entry" instead of "Add a Sample Entry." Some fields will be fixed (displayed in reverse video) and therefore inaccessible with the cursor. For example, the cursor begins in the data field "Completed," not in the data field "Tracking ID." Others will be presented in bold text, and so must be filled before the sample coordinator can go to the next screen. 


\title{
RML/Radiochemistry Sample Tracking Database
}

\author{
Add a sample Entry
}

Update a sample Entry

Search for a sample Entry

Due Date Check

\section{Iist Last Search Results}

\section{Exit from this Database Session}

\author{
Enter your choice $(A, U, S, D, L, E): U$ \\ 57 entries found; enter the number of the entry to update \\ or the tracking id of the entry to be retrieved:
}

Figure 13. Options Request Screen During Initiation of the Update Option.

\subsubsection{Search for a Sample Entry (User-Specified Searches)}

If the user does not know the sample tracking identification code for a sample to be updated, a search for the desired sample needs to be performed. Searches also prove useful when the user wants to collect several related sample entries. For example, the user may want to know how many samples have been submitted under a given charge number.

The user interface first presents the main screen displayed in Figure 15 when constructing a search. None of the fields in any of these data entry screens are fixed and none are required. The user can construct a simple or complex search. The user needs to keep in mind, though, that the search, in effect, retrieves the intersection of several individual retrievals. The search performs an "and" union, not an "or" set union. This can be viewed as follows:

(i) for the first filled data field, the search finds all entries that contain a match as field's 


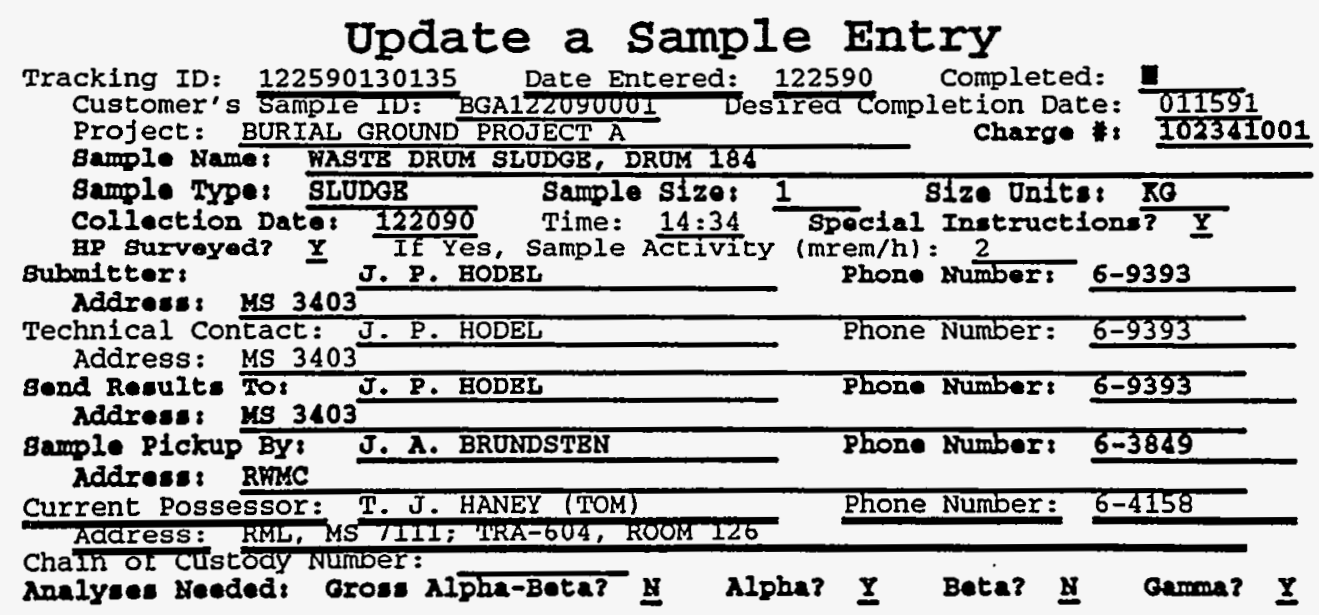

AZ when finished with screen. PF1 aborts program. PF2 displays help.

Flgure 14. A Main Screen for Updating a Sample Entry.

value; call these entries set number one

(ii) for the next filled data field, the search finds all entries that contain a match for this next field's value; call these entries set number two

(iii) those entries found in both sets become the new set number one

(iv) repeat steps ii and iii for the remaining filled data fields, and

(v) the final set number one contains the search results.

(In contrast, step iii for a search using an "or" set union would read "all the unique entries found in either set number one or set number two become set number one.")

There are times when providing less information is desirable. Be sure to consider if data entered is truly common to all the desired entries. It is generally advisable, though, to provide as much information as possible. This improves the accuracy of the search. While making the additional comparisons increases the response time, fewer extraneous sample entries will be retrieved. This makes it easier for the user to pick out the desired entry from the list.

The user interface presents the following information for each entry that it finds:

- the sample tracking identification code (SID)

- the customer's identification code (CID)

- the customer's sample name (CSN) and 


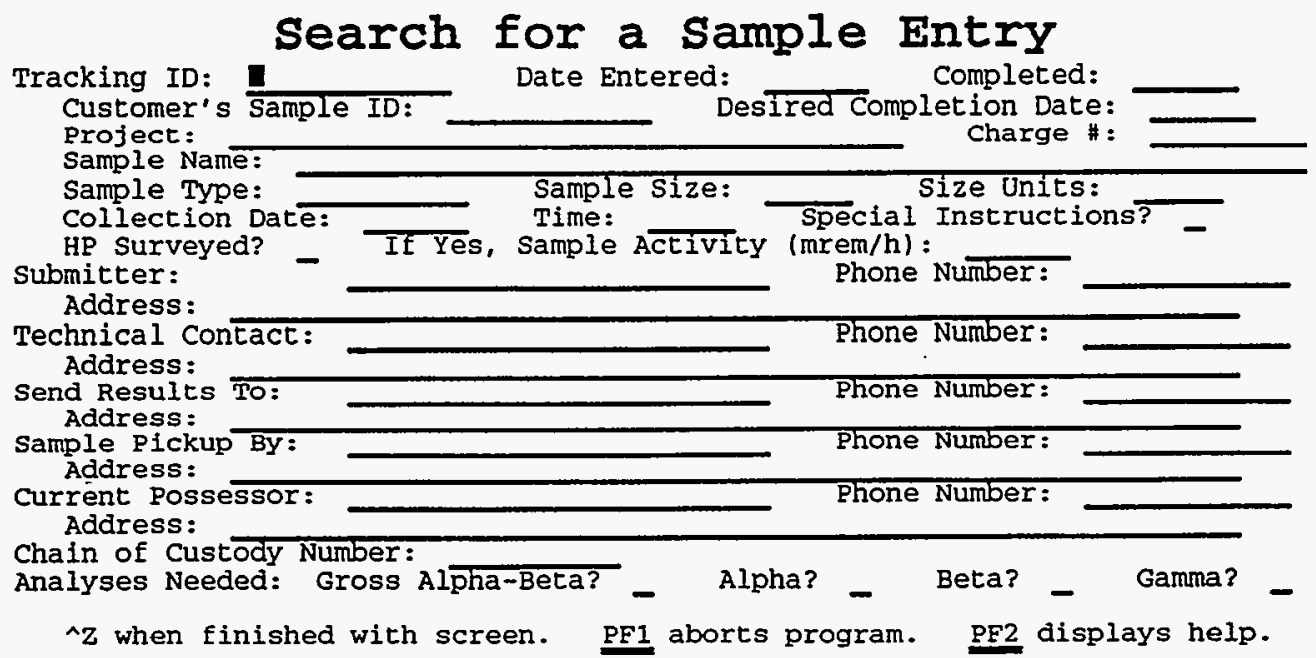

Figure 15. A Fresh Main Screen for Searching for a Sample Entry.

- the respective ordinal number within the list.

The entries are displayed in a fashion similar to the execution sequence tracking display. After the last data entry screen is completed, the user interface erases the display and begins listing the entries as they are found:

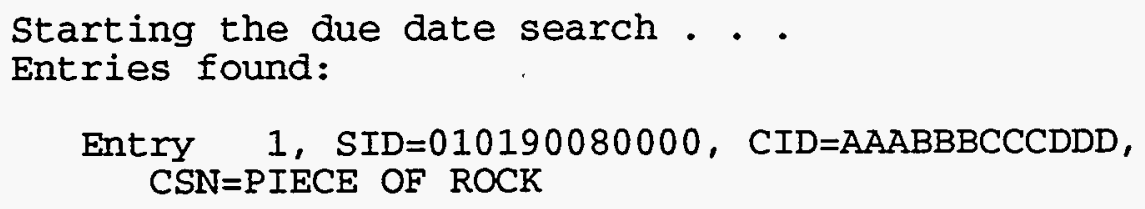

At the end of ' a full screen of entries, the user interface asks the user to strike the retum key before it continues displaying entries. This gives the user time to review the entries displayed. The user interface also waits until the user strikes any key after displaying the last entry found. The interface then returns to the options request screen.

The interface saves the information that is displayed. This information can be redisplayed by requesting a list of the last search results (option $L$ at the options request screen; see List Last Search Results, section 2.3.5). Entries from the list can be updated using the option "Update a Sample Entry" by entering the entry's ordinal number (see Update a Sample Entry, section 2.3.2).

\subsubsection{Due Date Check}

A specialized search provided by the user interface scans for analyses that are soon to be due (or are overdue). In this option the interface asks "How many days forward from today should be checked?" The user should enter a positive integer number. The interface then calculates the latest "Results needed by" date (or due date) to be scanned for. The target of the interface's search is all uncompleted analysis 
having a due date on or before this latest due date. The user interface presents the same information it does for a user-specified search (see Search for a Sample Entry, section 2.3.3) for each soon-to-be-due or overdue analysis it finds, along with the analysis that is due:

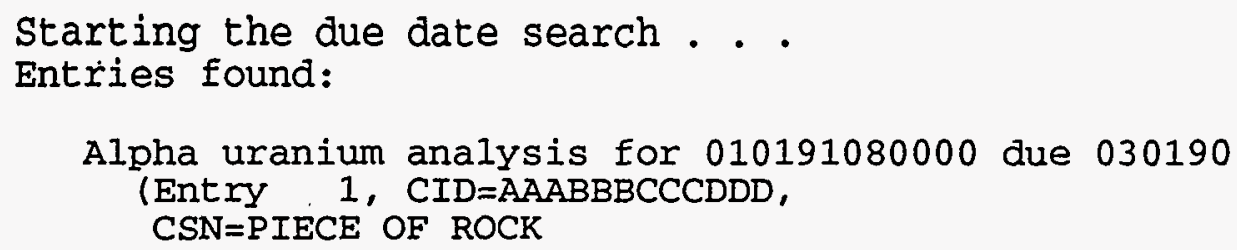

The user interface saves the same information that it does from a user-specified search; the analysis due is not saved. The listing generated can be used in the same ways a list from a user-specified search can be used.

\subsubsection{List Last Search Results}

The listing referred to here are the results from either a due date check or a user-specified search. The user interface saves the first one hundred entries from the most recent search. The interface displays the number of entries in the list, $\langle n\rangle$, at the bottom of the options request screen: " $<n>$ entries were found in the last search." If the list is empty, the user interface declares that "No search results are available!" when the user selects this option.

For a due date check, the list may not contain $<\mathrm{n}>$ unique sample entries. More than one analysis may be due for a given entry. Recall that the search results list from a due date check does not contain which analysis is due for a given entry.

\subsubsection{Exit from this Database Session}

To stop execution of the user interface, the user enters an "E" and then strikes the return key. The user interface clears the monitor display, the message "FORTRAN STOP" appears on the monitor, and the terminal session returns to the DCL prompt.

\subsection{Miscellaneous Notes}

\subsection{1 "When the nightmare comes to pass ..."}

There are two potentially unnerving occurrences for any user of the database. The following subsections cover these.

2.4.1.1 System Crashes. The nightmare: in the middle of a database session, the VAX mainframe computer crashes or is purposely shutdown. What is the user to do? Not a lot! If there are any wamings that a shutdown is imminent, the user should heed them and finish using the user interface as quickly as possible.

How worried should the user be? Not too much! The user interface minimizes the amount of time spent in communication with the database. Therefore, the likelihood of the computer going down while the interface is accessing the database is small. Also, there are some database self-repair mechanisms for dealing with improper shutdowns like a system crash. These execute automatically run 
when the computer comes back up. If the user is unable to access the database after a system crash, the database programmer should be notified.

What does the user stands to lose? Input to the user interface will be lost, such as data input to a screen when adding, updating, or searching for an entry. The list of sample tracking identification codes found in the last search of the database will be lost. The weekly user disk backup includes data already in the database. In the event of a catastrophic disk failure, at most the past week's additions and updates could be lost.

2.4.1.2 Database-Related Errors. The second nightmare: at some point the user receives this series of messages from the user interface:

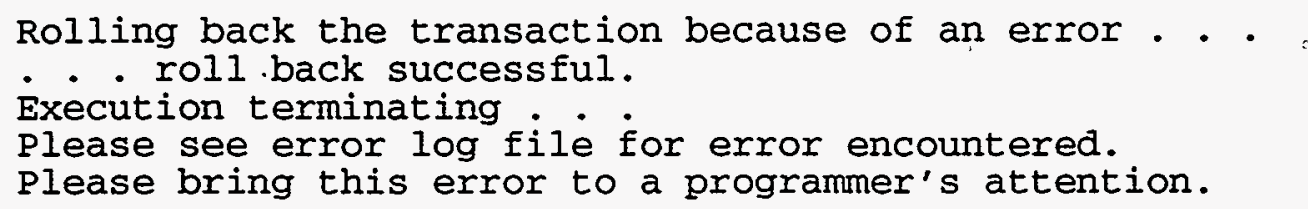

What happened? A database-related error occurred that the user interface does not know how to handle. Recall that, for example, the first two messages appear whenever the user asks to update a sample entry that does not exist. In this instance the user interface knows how to handle the error. It tells the user that an entry with the specified sample tracking identification code does not exist.

Whenever the user interface cannot handle a database error without terminating execution, it first rolls back the outstanding transaction. This undoes the outstanding (and probably incomplete or otherwise corrupt) transaction and returns the database to the pristine state it existed in before the transaction began. In contrast, when a transaction is committed, it has successfully completed without corrupting the database. Committed transactions cannot be subsequently rolled back, since committing a transaction ends the transaction, just as rolling it back does.

The user interface then writes the text of the error message to a file named SAMPLE_TRACKING_ERRORS.LOG. This file exists in the directory from which the user invoked the interface and is created anew each time the user interface starts. After successfully completing a database session, the user interface deletes the error $\log$ file.

If a session terminates because of an error, the given instructions should be followed. The user should print the screen containing the termination message. Also, it is preferable, though not required, that the user not invoke the interface until the condition that caused the error is resolved.

\subsubsection{Bar Code Labels}

A few points about the bar code labels are worth mentioning.

2.4.2.1 Affixing Bar Code Labels to Sample Containers. The bar code labels currently in use are self-adhesive. The label, the adhesive, and the printing on the label should prove long-lasting with little fading or discoloration and are resilient to most mild chemical reagents. To apply a label, peel it from the backing paper and press it onto the surface of the sample container.

1.; st of the containers used by the RML for making gamma-ray spectral measurements are cylindricai in shape. For these containers, affix the self-adhesive bar code label with its long edge parallel to the axis of rotation of the cylinder (see Figure 16). This will minimize the amount of curvature across 
the bar code label, allowing for easier reading by the scanner. The laser-diode bar code scanners can read bar codes through the yellow secondary-containment bags used in the RML, as long as the bag is smoothed over the bar code.
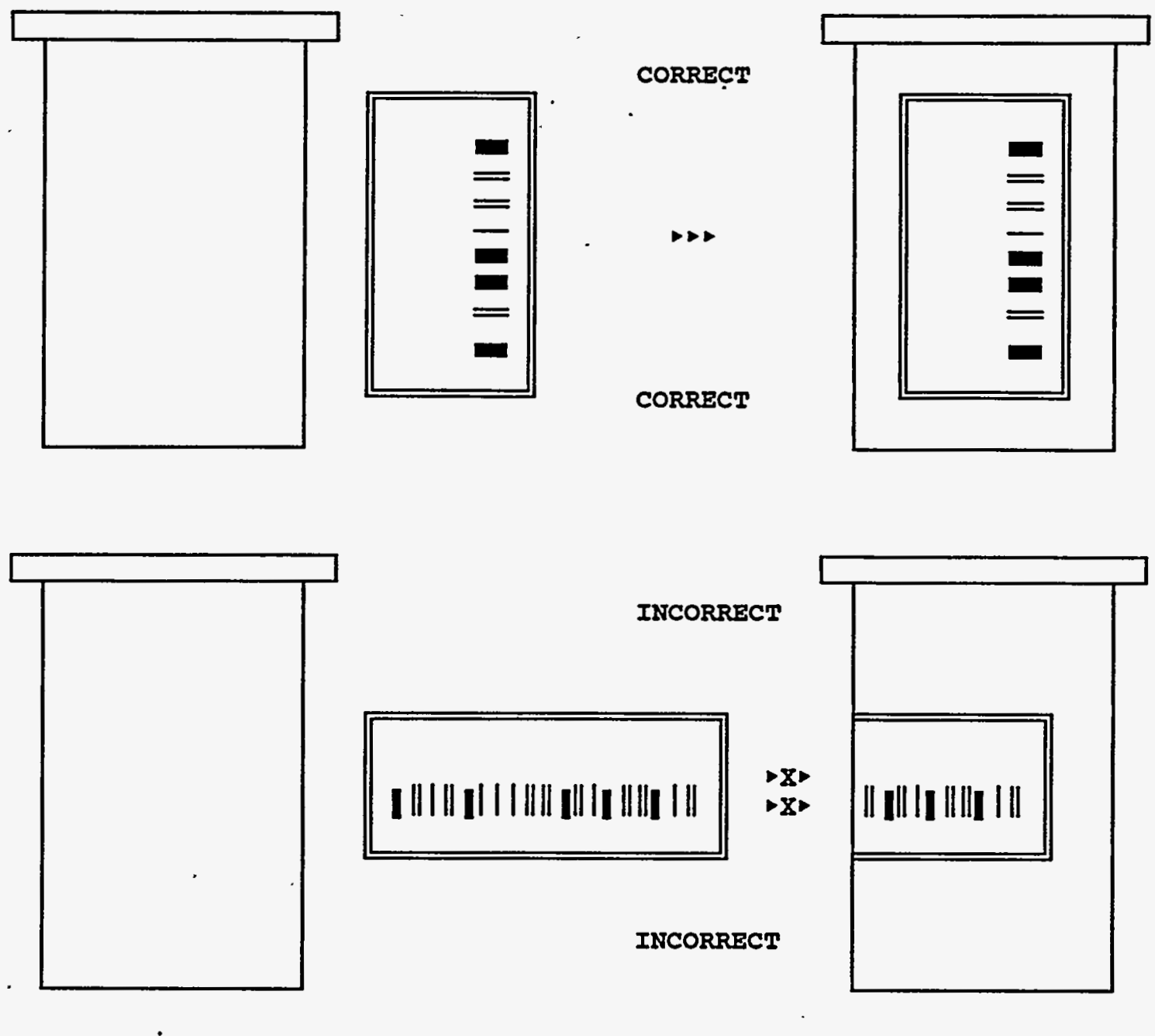

Figure 16. How to Affix Bar Code Labels to Cylindrical Sample Containers.

The remainder of the samples received by the RML are irregular in shape. For irregularly shaped sample containers, affix the bar code label to as smooth and as flat a portion of the sample container as is possible. A useful rule-of-thumb is that if the user cannot read the entire bar code label without moving the sample container, neither can the bar code scanner. For example, bar code labels should not be applied around the edge of an envelope. In this instance the user would have to tum the envelope over to see the other part of the label.

2.4.2.2 Replacing PrInter RIbbons and Label Rolls. The printer ribbons on the bar code printer should last through one roll of labels. Although the ribbon may be printing well, replace it each 
time an empty roll of labels is replaced. This will help to ensure that the printer produces a high-contrast, easily read label.

2.4.2.3 What if the bar code printer stops printing labels? The pattern for the bar code is stored in the bar code printer in a non-volatile RAM chip. This chip contains a battery which allows it to retain what is stored in its memory even if power to the printer is turned off. Nonetheless, this chip can sometimes lose the bar code pattem. (Note that this may indicate that the chip should be replaced; its intemal battery may be dead.)

If the pattem is lost, it needs to be down-loaded into the chip from a personal computer. An INTERMEC $\otimes$ Model $3000 \mathrm{~A}$ bar code label printer is currently in use by the RML, and so the compatible LabelWorks ${ }^{\mathrm{TM}}$ software is used to prepare the bar code pattern and down-load it to the printer. ${ }^{\mathrm{a}}$ Please refer to the appropriate manuals for how to prepare and down-load bar code patterns.

\subsubsection{What to do with Suggestlons or Comments on the Database or the User Interface}

Are the database and user interface doing what they need to do? Could either be improved, whether to upgrade performance, simplify use, or enhance presentation? Please feel free to present any suggestions or comments to the database programmer. The users are the primary resources in making the database and its user interface the best tools for the job.

a. This does not constitute an endorsement of INTERMEC 8 or LabelWorks ${ }^{\mathrm{TM}}$. Other bar code printers and software could also be used to generate the needed bar codes. 


\section{DESIGN OF THE DATABASE}

\subsection{Basic Description}

This database is a relational, multifile database. It is created and accessed using the Digital Equipment Corporation's Rdb/VMS implementation of the industry standard Structured Query Language (SQL). There is a separate file for each type of analyses and for basic sample information, tracking information, and special instructions. The directory [RDB_DATABASES.SAMPLE_TRACKING] on the user disk of the RML's VAX 6000 mainframe computer contains the database files. The weekly user disk backup includes the contents of these files (see Specifics, section 2.2).

The following subsections present a brief overview of the design of the database. The corresponding subsections under Specifics (section 2.2) present more detail. The presentation assumes the reader has some familiarity with relational database terminology.

\subsubsection{Tables and their Fields and Indexes for Sorting}

A table (or entity) contains a number of named fields for data storage. The names of the tables are as descriptive as possible while keeping the length of the name within the SQL-standard thirtycharacter limit.

Each field maps to only one data type (or domain). Appropriate fields serve as primary keys (for uniquely identifying each entry in a table) and foreign keys (for connecting entries between tables). Fields can have their own programmer-specified constraints (in addition to those of the corresponding domain) and comments. For example, null entries can be forbidden for a given field, such as the field that serves as the primary key.

A single field or a collection of fields serves as the primary key index for sorting each table. The name of this index is of the form <storage area name>_PRIMARY_KEY_INDEX. Its size depends on the fields which compose it. The comment for this index usually states the table for which it is the sorting key.

\subsubsection{Domains and Attributes}

A minimal set of data types, or domains, describes the space requirements for data in the database. Associated with each data type are a default value, constraints on the acceptable values, and a comment, all of which are programmer-specified.

Attributes are the data-containing fields within the database tables. Their names are as descriptive as possible while keeping the length of the name within the SQL-standard thirty-character limit. Each attribute's data type maps to only one domain.

\subsubsection{Storage Areas and Snapshot Files}

A separate storage area file contains each table and its definitions and constraints. The name of a storage area file is of the form <storage area name>.RDA. Its initial size is determined by the amount of data to be contained in a single entry to the table times an estimate of the number of entries that need to be available in the working database. The storage areas (and snapshot) files are automatically increased 
in size as needed by Rdb/VMS. (<storage area name> is a concatenation of the first two letters of each underscore-separated part of the table's name (and so can be computer-generated). For example, <storage area name $>$ for the table SAMPLE_INFORMATION is SAIN. The name of the table's snapshot file is of the form <storage area name>.SNP.)

\subsection{Specifics}

A listing of the SQL instruction set for creating this multifile database appears in Appendix A. The CREATE_SCHEMA tool generated this instruction set. (The software tools used to design the database are described in Reference 2. The examples presented in that report make use of this database.) The Lotus ${ }^{\circ}$ 1-2-3® print files, ${ }^{b}$ containing the entity- (table-) and screen-sorted attribute description lists, that serve as input to CREATE_SCHEMA appear in Appendix B. A recording of the terminal session from the execution of CREATE_SCHEMA, specifying the programmer's input for this database, appears in Appendix C.

The schema for this database is SAMPLE_TRACKING and so the name the root file is SAMPLE_TRACKING.RDB. The root file contains the complete set of domain and table definitions.

\subsubsection{Names of Tables, Storage Areas, and Snapshot Files}

The following are the table names, with the respective storage area names in parentheses:

- SAMPLE_INFORMATION (SAIN)

- for alpha analyses: ALPHA_URANIUM (ALUR), ALPHA_THORIUM (ALTH), ALPHA_PLUTONIUM (ALPL), ALPHA_AM241_SANS_PU239 (ALAMSAPU), ALPHA_AM241_WITH_PU239 (ALAMWIPU), ALPHA_TOTAL_SPECTROMETRIC (ALTOSP), and ALPHA_OTHER (ALOT)

- for gross alpha-beta analyses: GROSS_ALPHA_BETA_AIR_FILTERS (GRALBEAIFI) and GROSS_ALPHA_BETA_AIR_OTHER (GRALBEOT)

- for beta analyses: BETA_STRONTIUM_90 (BEST90), BETA_STRONTIUM_89_AND_90 (BEST89AN90), BETA_TOTAL_STRONTIUM (BETOST), BETA_TRITIUM (BETR), and BETA_OTHER (BEOT)

- for gamma analyses: GAMMA_SCREEN (GASC), GAMMA_FULL_ISOTOPIC (GAFUIS), and GAMMA_OTHER (GAOT)

- SPECIAL_INSTRUCTIONS (SPIN) and

- for possessors: TRACKING (TR).

\subsubsection{Names and Descriptions of Domains}

The following lists the domains and their respective descriptions. Many of the names fu!

b. The use of Lotus@ 1-2-3® in constructing this database does not constitute an endorsement of Lotus@ 1-23®. Other spreadsheet programs could also be used to generate these output files: 
describe the data type of the domain. For example, read "at most $\mathrm{X}$ alphanumerics" as the description when "X_CHARACTERS" is the name of the domain.

- CHARACTER_TIME_HH_MM (four or five numbers-plus-colon long and of the form hh:mm; for recording times-of-day)

- CHARGE_NUMBERS (exactly nine alphanumerics long)

- FIFTY_CHARACTERS

- FLAGS (one character long; for Yes/No data)

- FORTY_CHARACTERS

- IDENTIFICATION_CODES (exactly twelve alphanumerics long and of the numerical form mmddyyhhmmss; for the sample tracking database identification code)

- INTEGER_DATE_COUNT (date count starting at January 1, 1990; for use in "Due Date Check")

- INTEGER_NUMBERS

- NAMES (at most twenty-five alphanumerics long)

- PHONE_NUMBERS (at most twelve alphanumerics long)

- REAL_NUMBERS

-RML_SPECTRAL_ID_CODES (exactly fourteen alphanumerics long and of the form <save area abbreviation of two characters in length $><$ spectral identification code of eleven alphanumerics in length>, for the location and file name of spectral data; see Reference 1)

- SIXTEEN_CHARACTERS

- SIXTY_CHARACTERS

- TEN_CHARACTERS

- TWENTY_CHARACTERS

- UNITS (at most five alphanumerics long; for abbreviated units of measure such as $\mathbf{H}$ for hour, $\mathrm{M}$ for minute, $\mathrm{G}$ for gram, and $\mathrm{ML}$ for milliliter; note the exclusive use of uppercase letters, consistent with the user interface forcing all letters entered to uppercase)

- USER_NAME (at most thirty alphanumerics long; for tracking who last modified an entry) and

- VMS_DATE_TIME (seventeen or eighteen alphanumerics long and of the form "dd-mmm-yy hh:mm:ss"; for noting when a record was last modified).

\subsubsection{Backing Up the Database}


DCL BACKUP does not work correctly on the database files (the root file and storage area and snapshot files), owing to their intemal structure. These files are all marked for no backup using the DCL command SET FILE /NOBACKUP. This way the weekly backup of the user disk on the VAX 6000 mainframe computer does not include these files.

The weekly backup includes the contents of the database storage area files, though. The routine used by the RML operators to perform the backup contains code to do this (see Appendix D). The routine

(i) verifies the integrity of the database

(ii) performs an RMU BACKUP on the database (this a creates file than can be correctly saved and restored using DCL BACKUP

(iii) performs the DCL BACKUP

(iv) purges all but the two most recent versions of the file generated by RMU BACKUP and

(v) marks these files for no backup.

\subsubsection{Removal of Inactive Entries from the Database}

An inactive entry is one for which all work has been completed, say, at least six months ago. The inactive entries could be moved to a second, nearly identical version of the database. At a minimum, the inactive database would differ from the active database in that it would contain the date the entry was moved. The goal of removing inactive entries is to keep the active database as small as it needs to be; unneeded entries slow down searches and retrievals. The actual moving of entries can be performed automatically or by hand. It could be included as part of the backup procedure (see Backing Up the Database). Inactive entries could be made accessible to the user interface by allowing the user to specify the active or inactive database as the one to use. This capability does not exist with version 1.00 of the sample tracking database. 


\section{DESIGN OF THE USER INTERFACE}

\subsection{Basic Description}

Except for informational messages, the interface makes relating to the database transparent to the user. After selecting the desired option (Add a Sample Entry, Update a Sample Entry, .. .), the user fills in screen-displayed forms. The interface then constructs and executes the appropriate SQL calls. Screen management system routines are used for constructing the screen-displayed forms, reading data from them, and flagging entry errors and missing-yet-required information.

Owing to the case-sensitive nature of SQL searches, all alphabetic input to the interface program is forced to uppercase. Numerical input to data fields can be in the form of integer, real, or exponential numbers; the interface chooses the most complete and concise representation for displaying numerical data retrieved from the database.

\subsection{Specifics}

The software tools described in Reference 2 were used in preparing portions of code, such as declaration statements. They were also used to generate most of the dynamic SQL calls and related FORTRAN declaration and data-type conversion statements for the user interface. The examples presented in that report make use of this database.

The following subsections provide more detail on how the user interface stores data, converts data types, and performs error checking on entered data. It also describes the types of SQL calls the user interface uses and how, using SQL calls, it communicates with the database in performing insertions (for adding a sample entry), cursor declarations and fetches (for searching for a sample entry and due date checks), and modifications (for updating a sample entry). The routines and functions mentioned in the following subsections are described in more retail in-Reference 2.

\subsubsection{Data Storage Varlables and Arrays}

The array and scalar declaration statements (and some source code) related to the screen management routines are contained in the include file FLDDEF.INC (see Appendix E). These statements - were generated using the tools BUILD_SCREEN and CREATE_SCHEMA; the screen templates submitted to BUILD_SCREEN are presented in Appendix F (see Reference 2).

4.2.1.1 Storage of Data. For ease of use with screen management routines, all information for a given screen is saved in a character array (composed of eighty-character-long elements) assigned to that screen. Since SQL calls cannot make use of arrays (and variable names over six characters long are discouraged in $\mathrm{Rdb} / \mathrm{VMS}$ ), the arrays are equivalenced to a set of scalar variables that are of the required length for the appropriate domains. A character array's name is of the form fidat<first letter of the screen's name>; for example, for the Main screen the array's name is fidatM.

BUILD_SCREEN creates a set of scalar variables using a generic naming scheme that only identifies the screen. For example, scalar variables for the main screen's data fields are named fldM $<\mathrm{n}\rangle$, where $\langle\mathrm{n}\rangle$ is the sequential number of the field within the screen. For clarity, the names of the scalar variables were changed to include some relation to the appropriate attribute.

The new names of the scalar variables begin with $f<$ first letter of the screen's name>. The 
remaining four letters, at most, of each name are constructed in a fashion similar to how storage area names are constructed (see Storage Areas and Snapshot Files, section 2.1.3) but using only the first letter of each underscore-separated part of the respective attribute. For example, for the main screen's sample tracking identification code (attribute name SAMPLE_TRACKING_ID) the scalar variable is named fMSTI (Main screen, then SAMPLE, then TRACKING, then ID). (Some hand modifications were required to eliminate duplicate names).

4.2.1.2 Conversion of Character Data to Numerical Data. If the database requires a number for a given attribute, the appropriate translation of character-to-integer or character-to-real is performed. (The result is stored in a scalar variable the name of which begins with an "i" instead of an " $\mathrm{f}$ " for integer data and with an " $\mathrm{r}$ " instead of an " $\mathrm{f}$ " for real data). The reverse translation is performed for numerical data retrieved from the database and to be displayed via screen management routines. These conversions are handled by the FORTRAN functions RVALUE (for character string-to-real number conversions) and CVALUE (for real number-to-character string conversions). The codes for these functions, again, are presented in Reference 2.

The arrays containing labels for the fields have names of the form field<first letter of the screen's name>. The arrays containing the names of the entities and attribute corresponding to the screen fields have names of the form atrbt<first letter of the screen's name>. There are no scalar equivalents for these arrays. Other arrays and scalars used in the screen management calls are similarly constructed; Table 1 lists all the arrays associated with any screen. (Note that from here on and in the table "<first letter of the screen's name $>$ " is abbreviated " $\diamond$. ")

Some of these arrays will be discussed in more detail later, in the following subsection on error checking for example. The implementation of these arrays, and the coding for the error checking, is found in the FORTRAN routine DISPLY (see Reference 2).

4.2.1.3 Error Checking. The user interface checks for three types of errors. First, is the data entered short enough to fit into the appropriate field? Each data field has a maximum number of alphanumerics that can be entered into it, mxnoc $\diamond$. Second, is the data required? If the field is rendered in bold print, an entry must be made into the data field before the user can leave that screen. Also, an entry can be required in a field because an entry has been made in a related field: if an entry appears in the "Date Completed" field, for example, an entry would be required in the "Results report citation" field. Third, is the data entered of the type specified by the fityp $>$ array? While data entered into a field is read into a FORTRAN CHARACTER variable, a real number may be needed for the corresponding attribute in the database. The following data types, as implemented in the FORTRAN routine DISPLY, are presently available.

4.2.1.3.1 "a" for Anything - Any combination of alphanumerics, spaces included and up to the specified maximum length, is allowed.

4.2.1.3.2 " $d$ " for Date - A date in the integer form mmddyy is allowed. The entry will be converted into an integer date count (that is, how many days have elapsed between the specified date and December 31,1989 ) for storage in the database. Integer date counts make due date checks easier to perform, for instance. Conversions are accomplished using the FORTRAN functions DAYCNT (character mmddyy-to-integer date count) and CHRDAT (integer date count-to-character mmddyy).

An entry of this type must be composed of exactly six integers (leading and trailing zeros must be included). The first two digits, $\mathrm{mm}$, represent the month and must fall in the range 01 to 12 (inclusive). The second two digits, dd, represent the day of the month. The last two digits, yy, represent the 
Table 1. Arrays and Scalars Used in Coding the User Interface.

\begin{tabular}{|c|c|c|c|}
\hline Array Name & Description & $\begin{array}{c}\text { Scalar } \\
\text { Equivalent? }\end{array}$ & $\begin{array}{l}\text { Letter for } \\
\text { Equivalent }\end{array}$ \\
\hline field $>$ & contents of the description field & $\mathbf{N}$ & - \\
\hline fidat $>$ & $\begin{array}{l}\text { contains error-free data (or is null) from the } \\
\text { data field }\end{array}$ & $\mathbf{Y}$ & f \\
\hline atrbtes & $\begin{array}{l}\text { table (entity) and attribute names corresponding } \\
\text { to the screen field }\end{array}$ & $\mathbf{N}$ & - \\
\hline varble & $\begin{array}{l}\text { contains the names of scalar variables equiva- } \\
\text { lenced to fidat }>\text { and to the respective if-null } \\
\text { indicator }\end{array}$ & $\mathrm{N}$ & - \\
\hline fityp $>$ & $\begin{array}{l}\text { allowed data type for the data field ("a" for } \\
\text { alphanumeric, e.g.) }\end{array}$ & $\mathbf{N}$ & - \\
\hline fdlen $>$ & length of the description field & $\mathrm{N}$ & - \\
\hline fdrow $>$ & $\begin{array}{l}\text { row number for display of the description and } \\
\text { data fields }\end{array}$ & $\mathbf{N}$ & - \\
\hline fdcolo & $\begin{array}{l}\text { starting column number for display of the } \\
\text { description field }\end{array}$ & $\mathbf{N}$ & - \\
\hline ficol $>$ & $\begin{array}{l}\text { starting column number for display of the data } \\
\text { field }\end{array}$ & $\mathrm{N}$ & - \\
\hline mxnoc $>$ & $\begin{array}{l}\text { maximum number of alphanumerics allowed in } \\
\text { the data field (length of data field) }\end{array}$ & $\mathbf{Y}$ & $\begin{array}{c}1 \\
\text { (for length) }\end{array}$ \\
\hline fdrndo & $\begin{array}{l}\text { rendition of the description field (normal if } \\
\text { optional, bold if required, reverse video if } \\
\text { fixed) }\end{array}$ & $\mathbf{N}$ & - \\
\hline firnd $>$ & rendition of the data field & $\mathbf{N}$ & - \\
\hline ifnul & $\begin{array}{l}\text { if-null indicator: }-1 \text { if the data field is empty } \\
\text { (null), } 0 \text { otherwise }\end{array}$ & $\mathbf{Y}$ & $\stackrel{n}{n}$ (for null) \\
\hline ifrqdo & $\begin{array}{l}\text { entry into the current data field required if data } \\
\text { field number ifrqd }>\text { is not empty }\end{array}$ & $\mathbf{N}$ & - \\
\hline idito $\infty$ & $\begin{array}{l}\text { "ditto" transfers contents of data field number } \\
\text { idito }>\text { into the current data field }\end{array}$ & $\mathbf{N}$ & - \\
\hline ifmodo & $\begin{array}{l}\text { if-modified indicator: true if the data field's } \\
\text { value has been changed (during Update), false } \\
\text { otherwise }\end{array}$ & $\mathbf{Y}$ & $\stackrel{\mathrm{m}}{\text { (for modified) }}$ \\
\hline
\end{tabular}

year and are interpreted as follows: if yy is between 90 and 99, the year is 19yy; if yy is between 00 and 
79, the year is 20yy. Entries between 80 and 89 are not allowed as they should be interpreted as 20yy, yet the resulting integer date count would be too large to fit into a FORTRAN INTEGER*4 variable and hence into the respective database attribute.

4.2.1.3.3 "I" for Length - Here the entry must be exactly mxnoc $>$ alphanumerics long. There are no restriction as to the alphanumerics used. One instance in which "l" is used is the charge number to be billed for performing the analyses. All charge numbers are exactly nine alphanumerics long, so a shorter or longer entry is invalid.

4.2.1.3.4 " $t$ " for Time - The format of the entry allowed here is either "h:mm" or "hh:mm." $\mathrm{h}$ or hh represents the hour as an integer. A leading zero is not necessary because the colon can be used to delineate the hour portion of the entry from the minute portion. $\mathrm{mm}$ represents the number of minutes.

4.2.1.3.5 "u" for Units of TIme - In instances such as the units for the duration of the spectral acquisition, an entry of either $\mathrm{H}$ for hours or $\mathrm{M}$ for minutes is allowed. " $\mathrm{u}$ " covers only units of time (duration); it does not cover sample size units, for example.

4.2.1.3.6 "?" for Questlons needing a Yes-or-No Answer - The only allowed entries here are " $\mathrm{Y}$ " for yes and "N" for no. This data type is used for flags such as whether a certain type of analysis is to be performed.

\subsubsection{Communication between the User Interface and the Database}

SQL calls are used to transfer data between the user interface and the database. These calls are either hard-coded into the user interface (precompiled SQL) or are constructed by it (dynamic SQL).

4.2.2.1 Forms of SQL Used. Both precompiled SQL and dynamic SQL calls are used. Precompiled SQL calls are completely known by the user interface before they are executed. Such calls include inserting all attributes of an entry into the database. An EXEC SQL preface marks these calls as precompiled SQL statements rather than as statements in the host program's language.

Dynamic SQL calls can only be composed during execution of the user interface. Instances of these include requesting the tables needed for the current transaction. The instruction set for a dynamic SQL call is constructed as a FORTRAN CHARACTER variable and is executed from the main routine using EXEC SQL EXECUTE IMMEDIATE. To minimize the length of the character string, a FORTRAN routine APSTRG (APpend character STRinG) fills the character variable with each additional portion of the SQL call passed to it.

Modular SQL, in which calls are made from the main routine to SQL routines constructed in a single and separate file, is not used. The main routine of the user interface is larger than might seem prudent for ease of code development because of the Rdb/VMS requirement that all precompiled SQL statements reside in the same routine.

4.2.2.2 Commencing a Database Transaction. Communications with the database occur within transactions. The transaction definition (SET TRANSACTION) specifies the tables of the database the user needs and the type of access required. For example, for adding an entry to the database, the sample coordinator needs exclusive write access to all of the tables for the requested analyses. No one else should be able to access these tables in any fashion until the addition is completed. The construction of the SET TRANSACTION call depends on the application (for example, see Add a Sample Entry, 
section 3.2.2.3).

At present, all SET TRANSACTION calls will wait at most sixty seconds for the requested tables to become available. To minimize conflicts (lock conditions resulting from two users wanting the same table at the same time and in incompatible fashions), database transactions last only as long as necessary. Any database transaction starts only as soon as all the requisite information is available. Again using the example of adding an entry, the database transaction does not begin until all of the appropriate screens are filled by the user. And, accordingly, each transaction is closed - either committed, that is, saved, or rolled back, that is, undone - upon completion.

4.2.2.3 Add a Sample Entry. The addition (insertion) of a sample entry to the database occurs via the SQL INSERT command. Here the main routine constructs the SET TRANSACTION command (using the FORTRAN routine APSTRG mentioned above in Forms of SQL Used), and then executes it as a dynamic SQL call (EXECUTE IMMEDIATE). Again, dynamic SQL is used since all of the tables that will be needed are generally not known until it is time for the addition to occur. All tables needed are included in a single SET TRANSACTION command because violations of constraints are better managed within a single transaction than over a series of transactions. A constraint, for example, may require that an entry for sample identification code exists in the sample information table before an entry for that same identification code can be made into an analysis table. A typical sequence of calls to APSTRG, followed by execution of the SET TRANSACTION call, would be

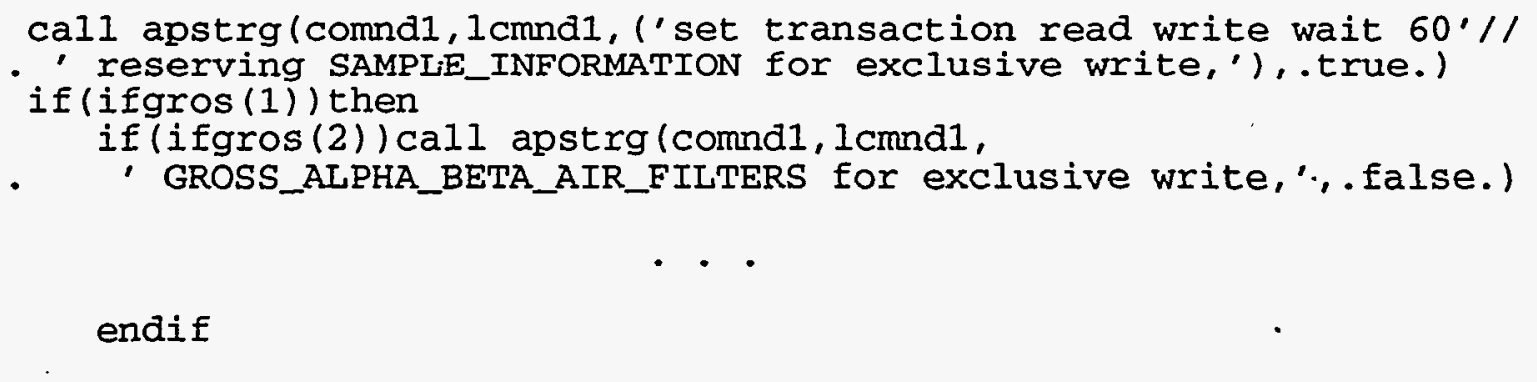

call apstrg (comnd1, Icmnd1,

- (' SPECIAL_INSTRUCTIONS for exclusive write, '//

- 'TRACKING for exclusive write'), . false.)

exec sql execute immediate :comndl

The next SQL calls are precompiled SQL calls and insert the data provided by the sample coordinator into the database:

exec sql insert into SAMPLE_INFORMATION values (

- : fMSTI: $N M S T I,:$ iMDSE : NMDSE, : IMDAWC: NMDAWC, : IMCSI: NMCSI,

- : iMRNBD: nMRNBD, : fMCPN: nMCPN, : fMWCN : nMWCN, : fMCSN:nMCSN,

- : fMST:nMST, : rMSS :nMSS, : fMSSU:nMSSU, : iMSCD:nMSCD,

- : :MSCT:nMSCT, : fMASI : nMASI, : fMSHS : nMSHS, :rMSHSA :nMSHSA,

- : fMSSN: nMSSN, : fMSSPN: nMSSPN, : fMSSA:nMSSA, : fMTCN: $n M T C N$,

- : IMTCPN: nMTCPN, : FMTCA: IMTCA, : IMSRTN: nMSRTN, : fMSRTP : nMSRTP,

- : : MSRTA : nMSRTA, : fMSPBN : $n M S P B N$, : fMSPBP : nMSPBP, : fMSPBA : nMSPBA,

- : fMNGAB : nMNGAB , : fMNA : nMNA, : IMNB : nMNB , : fMNG : nMNG, 
- :nxinst : ifnnxi, :nxpss 1 : ifnnxp, : fGNGS:nGNGS, : fGNGFI :nGNGFI,

- : fGNGO:nGNGO, : fCNGAF :nCNGAF, : fCNGAO:nCNGAO, : fBNBS $r$ :nBNBSr,

- : $\quad$ fBNBSC:nBNBSC, : fBNBST:nBNBST, : $\mp B N B T: n B N B T,: £ B N B O: n B N B O$,

- : fANAU : nANAU, : fANATh: nANATh, : fANAPu: nANAPu, : fANASP: nANASP,

- : fANAWP : nANAWP, : fANATS : nANATS, : fANAO:nANAO, USER, : todaY: notnul,

- $\quad$ IMCOCN $:$ nMCOCN)

The values are passed in the form :<scalar equivalent of data field $>$ :<scalar equivalent of if-null indicator $>$ The colon preceding each variable name tells the SQL precompiler that what follows is a variable in the user interface's native language, not an SQL variable or key word.

What about fields into which no entry was made? Recall that there is an if-null indicator array. If a field is left blank, that field's if-null indicator is set (to a value of -1). When this if-null indicator is passed to the database (with a value of -1), the attribute in the database entry is marked NULL. (The ifnull indicator could be used to specify which attributes are to be passed to a table. Dynamic SQL would be used in place of precompiled SQL in this case. Pointers to variables are required in place of variables for dynamic SQL, though.)

4.2.2.4 Search for a Sample Entry. This is another instance where dynamic SQL calls could be used; this time they are, in conjunction with the APSTRG routine and the atrbt $\triangle$ and fidat $\triangle$ arrays. To perform a search, the user fills in all the fields necessary to describe the entry or entries desired. The if-null indicator is used to indicate the fields to be included in the search command. The user is to leave blank those fields for which no information is known or for which the information may vary among the desired entries.

Recall that the atribt $>$ arrays contain the name of the table (entity) and attribute that correspond to a given screen field. The search command is constructed using the atrot $>$ arrays and the values contained in the fidat $>$ arrays. If a data field contains an entry (and so its if-null indicator is not set), the appropriate elements from atrot $\triangle$ and the entry are used in constructing the dynamic SQL search call via APSTRG. These portions of the search command are constructed in the FORTRAN routine SRCBLD (SEARCH BUILD).

Searches are conducted using cursors and fetches from cursors. A cursor can be thought of as a virtual table containing only the specified entries (EXEC SQL DECLARE . . . CURSOR for <statement name>; EXEC SQL PREPARE <statement name> from <search command>). Data from a cursor can be retrieved into native language variables (EXEC SQL FETCH . . . INTO . . .). A typical portion of the main routine's code for constructing the search command and executing the search and retrieval is

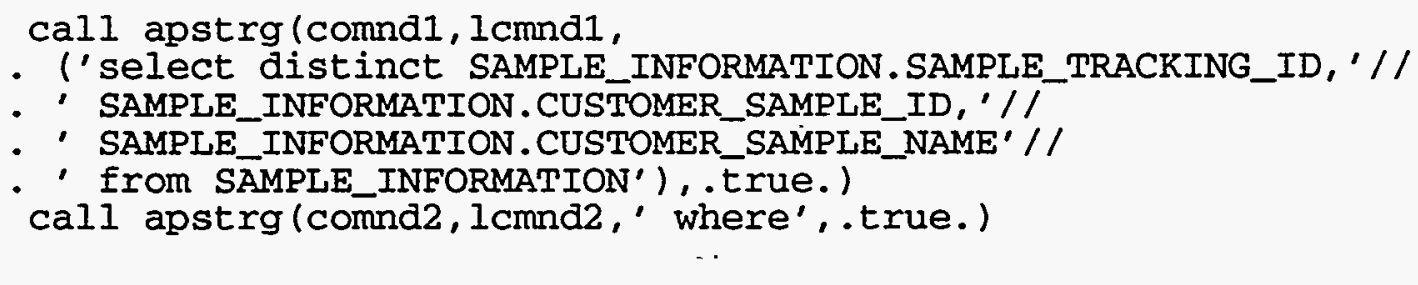

call srcbld (comnd1, lcmnd1, comnd2, lcmnd2, numfdM, fidatM, mxnocM, - fitypM, atrbtM)

if (ifgama (1)) call srcbld (comnd1, lcmnd1, comnd2, lcmnd2, numfdG,

- fidatG, mxnocG, fitypg, atrbtG) 
cal1 apstrg (comnd1, 1 cmnd1, (comnd2 ( $1: 1$ cmnd2)//

- ' order by SAMPLE_INFORMATION.SAMPLE_TRACKING_ID asc'),.false.)

exec sql declare SEARCH_cursor cursor for SEARCH_statement

exec sql prepare SEARCH_statement from comndl

exec sql open SEARCH_cursor

exec sql whenever not found goto 704

702 . . .

exec sql fetch SEARCH_cursor into :smplid,:custid,:csname

goto 702

exec sql close SEARCH_cursor

Note that two character strings are input to the routine APSTRG. For each non-null data field, a call to SRCBLD transfers the name of the respective entity alone to the first string (if it does not already appear there) and, to the second string, the entity (table) name, the attribute name, and the value contained in the data field. The second string is appended to the first string prior to the search. Finally, the search is performed as the cursor is declared (defined), opened (much as a file is opened), and read from (via FETCH) until the last entry is found (watched for by WHENEVER NOT FOUND . . .). The data retrieved can be displayed as it is found and stored for future reference. After all the data is retrieved from the cursor, the cursor is closed.

4.2.2.5 Due Date Checks. Due date checks are a form of search where the same, specific attributes in the various tables are always interrogated, namely the date-completed fields. The sample coordinator states how many days ahead should be checked for analyses and reports which will be due. The user interface calculates the integer date count for the current day, and adds to it the number of days ahead to be checked. A search is then preformed for all entries having due dates the integer date count for which is less than or equal to the above sum (IDUCNT) and for which the specified work has not been completed (that is, for which the DATE_ALL_WORK_COMPLETED field is null). The coding for one such search is

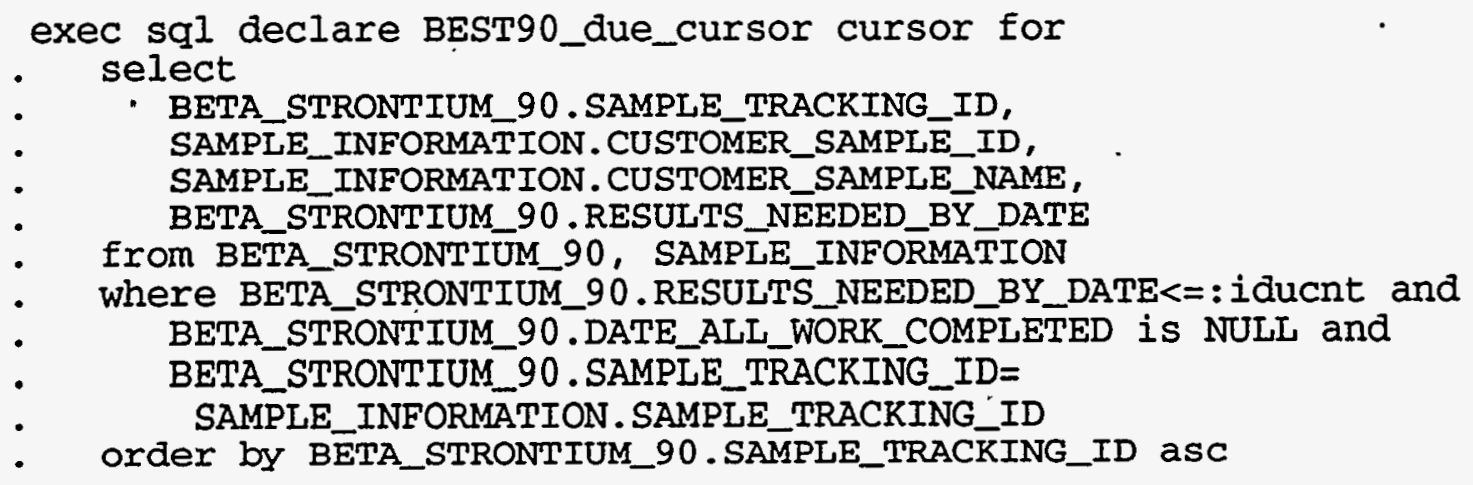


exec sql open BEST90_due_cursor

exec sql whenever not found goto 836

834 exec sql fetch BEST90_due_cursor into :smplid,:custid, :csname, - : duedat

836

goto 834

exec sql close BEST90_due_cursor

4.2.2.6 Update a Sample Entry. This final mode of communication uses the techniques described above. First, data for the specified entry is fetched from the database via a cursor:

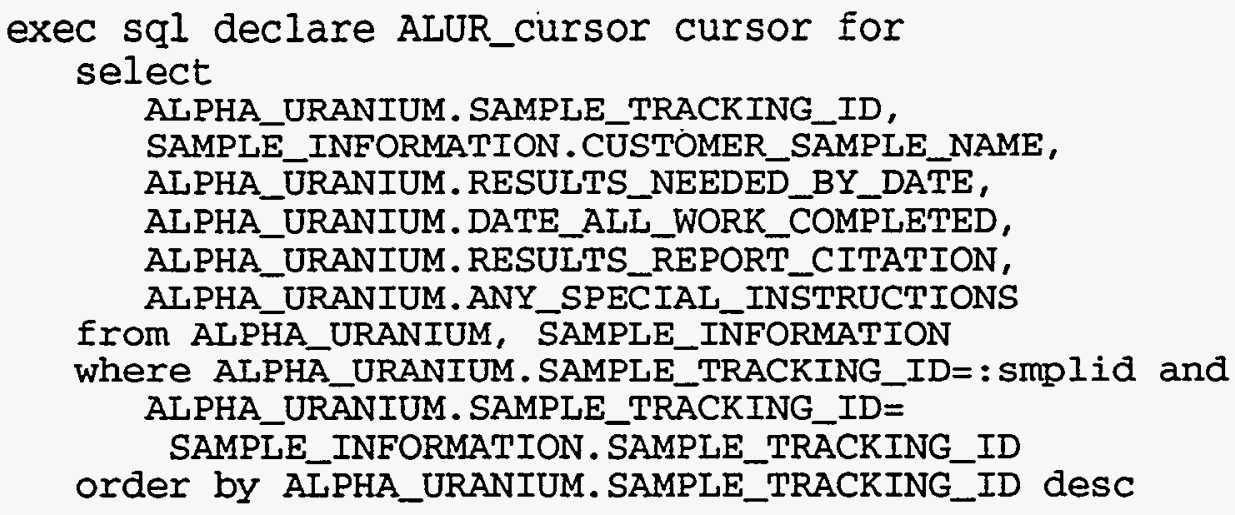

exec sql open ALUR_cursor

exec sql fetch ALUR_cursor into

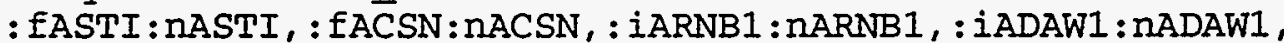

: fARRC1 : $\mathrm{AARRC1}$, : AASI1 : $\mathrm{AAASI1}$

exec sql close ALUR_cursor

The retrieved data is then displayed for the sample coordinator to update. Once the updating of the screens is completed, the entire database entry is updated (modified) if any changes have been made within the screens.

The if-modified indicator plays a role different from that the if-null indicator plays in searching for a sample entry. If any of the if-modified indicators for a table's fields are true, the entire entry is updated if it previously existed in the database. If the entry is new to the database, the entry is added to the database: 
if (ifalph (2) . and. (MARNB1. or .MADAW1. or .MARRC1. or .MAASI1)) then

if(ifalpo(2)) then !Use update to change the entry. exec sql update ALPHA_URANIUM set

RESULTS_NEEDED_BY_DATE=: IARNB1:nARNB1, DATE_ALI_WORK_COMPLETED $=$ : IADAW1 : NADAW1, RESULTS_REPORT_CITATION=: IARRC1 : IARRC1, ANY_SPECIAL_INSTRUCTIONS=: AAASI1 : AAASI1, USER_CREATING_OR_MODIFYING=USER, DATE_CREATED_OR_MODIFIED $=:$ today:notnul where SAMPIE_TRACKING_ID=: Smplid

else !Use insert since it was not there before. exec sql insert into ALPHA_URANIUM values ( : fASTI: nASTI, : iARNB1: nARNB1, : iADAW1 : nADAW1, endif : fARRC1:nARRC1, : fAASI1:nAASI1,USER, : today:notnul) endif 


\section{REFERENCES}

1. E. W. Killian and D. A. Femec, Operator's Guide for VAXGAP, a Gamma-Ray Spectrum Analysis Package, EGG-2672, August 1992.

2. D. A. Femec, Version 1.00 Programmer's Tools Used in Constructing the INEL RML/Analytical Radiochemistry Sample Tracking Database and its User Interface, INEL-95/0454, September 1995. 


\section{APPENDIXES}

\section{A. SQL INSTRUCTION SET FOR CREATION OF SAMPLE TRACKING DATABASE}

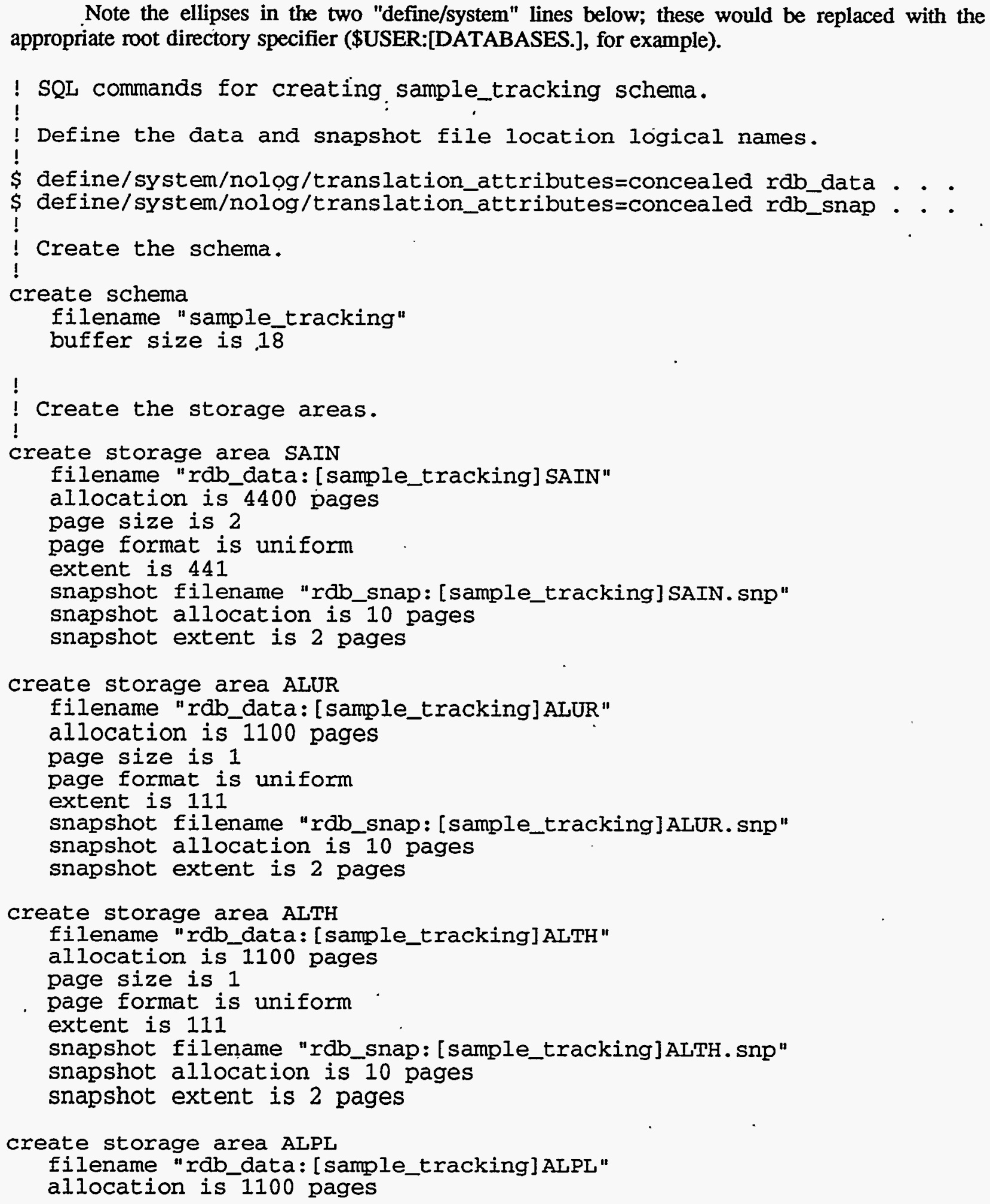

Note the ellipses in the two "define/system" lines below; these would be replaced with the appropriate root directory specifier (\$USER:[DATABASES.], for example).

! SQL commands for creating. sample_tracking schema. ! Define the data and snapshot file location logical names.

\$ define/system/nolog/translation_attributes=concealed rdb_data . . . \$define/system/nolog/translation_attributes=concealed rdb_snap . . . 
page size is 1

page format is uniform

extent is 111

snapshot filename "rdb_snap: [sample_tracking]ALPL.snp"

snapshot allocation is 10 pages

snapshot extent is 2 pages

create storage area ALAMSAPU

filename "rdb_data: [sample_tracking] ALAMSAPU"

allocation is 1100 pages

page size is 1

page format is uniform

extent is 111

snapshot filename "rdb_snap: [sample_tracking]ALAMSAPU.snp" snapshot allocation is 10 pages

snapshot extent is 2 pages

create storage area ALAMWIPU

filename "rdb_data: [sample_tracking] ALAMMIPU"

allocation is 1100 pages

page size is 1

page format is uniform

extent is 111

snapshot filename "rdb_snap:[sample_tracking] ALAMWIPU.snp" snapshot allocation is 10 pages

snapshot extent is 2 pages

create storage area ALTOSP

filename "rdb_data: [sample_tracking] ALTOSP"

allocation is 1100 pages

page size is 1

page format is uniform

extent is 111

snapshot filename "rab_snap:[sample_tracking]ALTOSP.snp"

snapshot allocation is 10 pages

snapshot extent is 2 pages

create storage area ALOT

filename "rdb_data: [sample_tracking] ALOT"

allocation is 1100 pages

page size is 1

page format is uniform

extent is 111

snapshot filename "rdb_snap: [sample_tracking]ALOT.snp"

snapshot allocation is 10 pages

snapshot extent is 2 pages

create storage area BEST90

filename "rdb_data:[sample_tracking] BEST90"

allocation is 1100 pages

page size is 1

page format is uniform

extent is 111

snapshot filename "rdb_snap: [sample_tracking]BEST90.snp"

snapshot allocation is 10 pages

snapshot extent is 2 pages

create storage area BEST89AN90

filename "rdb_data: [sample_tracking] BEST89AN90"

allocation is 1100 pages 
page size is 1

page format is uniform

extent is 111

snapshot filename "rdb_snap:[sample_tracking]BEST89AN90.snp"

snapshot allocation is 10 pages

snapshot extent is 2 pages

create storage area BETOST

filename "rdb_data: [sample_tracking] BETOST"

allocation is 1100 pages

page size is 1

page format is uniform

extent is 111

snapshot filename "rab_snap: [sample_tracking] BETOST.snp"

snapshot allocation is 10 pages

snapshot extent is 2 pages

create storage area BETR

filename "rdb_data: [sample_tracking] BETR"

allocation is 1100 pages

page size is 1

page format is uniform

extent is 111

snapshot filename "rdb_snap:[sample_tracking]BETR.snp"

snapshot allocation is 10 pages

snapshot extent is 2 pages

create storage area BEOT

filename "rdb_data: [sample_tracking]BEOT"

allocation is 1100 pages

page size is 1

page format is uniform

extent is 111

snapshot filename "rdb_snap:[sample_tracking] BEOT.snp"

snapshot allocation is 10 pages

snapshot extent is 2 pages

create storage area GASC

filename "rdb_data: [sample_tracking] GASC"

allocation is 4400 pages

page size is 1

page format is uniform

extent is 441

snapshot filename "rdb_snap: [sample_tracking]GASC.snp"

snapshot allocation is 10 pages

snapshot extent is 2 pages

create storage area GAFUIS

filename "rdb_data: [sample_tracking] GAFUIS"

allocation is 4400 pages

page size is 1

page format is uniform

extent is 441

snapshot filename "rdb_snap:[sample_tracking]GAFUIS.snp"

snapshot allocation. is 10 pages

snapshot extent is 2 pages

create storage area GAOT

filename "rdb_data: [sample_tracking] GAOT"

allocation is 4400 pages 
page size is 1

page format is uniform

extent is 441

snapshot filename "rdb_snap: [sample_tracking]GAOT.snp"

snapshot allocation is 10 pages

snapshot extent is 2 pages

create storage area GRALBEAIFI

filename "rdb_data: [ sample_tracking] GRALBEAIFI"

allocation is 1100 pages

page size is 1

page format is uniform

extent is 111

snapshot filename "rdb_snap:[sample_tracking]GRALBEAIFI.snp"

snapshot allocation is 10 pages

snapshot extent is 2 pages

create storage area GRALBEOT

filename "rdb_data: [sample_tracking] GRALBEOT"

allocation is 1100 pages

page size is 1

page format is uniform

extent is 111

snapshot filename "rdb_snap: [sample_tracking] GRALBEOT.snp"

snapshot allocation is 10 pages

snapshot extent is 2 pages

create storage area SPIN

filename "rdb_data: [sample_tracking] SPIN"

allocation is 1466 pages

page size is 6

page format is uniform

extent is 147

snapshot filename "rab_snap:[sample_tracking]SPIN.snp"

snapshot allocation is 10 pages

snapshot extent is 2 pages

create storage area TR

filename "rdb_data: [sample_tracking] TR"

allocation is 4400 pages

page size is 3

page format is uniform

extent is 441

snapshot filename "rdb_snap:[sample_tracking]TR.snp"

snapshot allocation is 10 pages

snapshot extent is 2 pages

;

! Create the domains, including defaults, constraints, and comments.

!

create domain USER_NAME char(30)

default NULL;

comment on domain USER_NAME

is 'Domain for user name.';

create domain VMS_DATE_TIME char(18)

default NULL; 


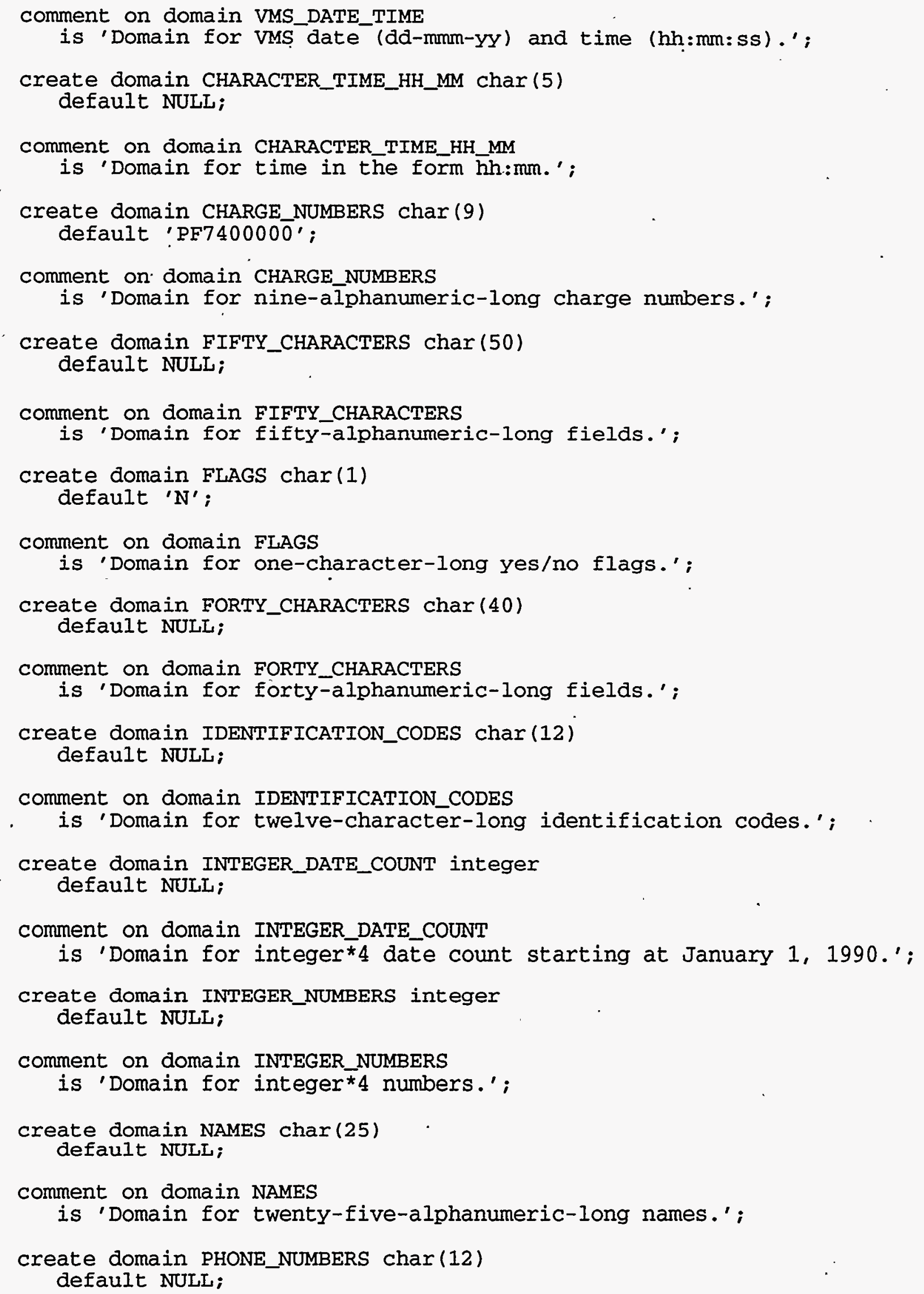


comment on domain PHONE_NUMBERS

is 'Domain for twelve-alphanumeri=-Iong phone numbers.';

create domain REAL_NUMBERS real

default NULL;

comment on domain REAI_NUMBERS

is 'Domain for real* 4 numbers.';

create domain RML_SPECTRAL_ID_CODES char(14)

default NULL;

comment on domain RML_SPECTRAL_ID_CODES

is 'Domain for fourteen-character-long $\mathrm{RML}$ spectral identification

codes (<save area>\$<spectral id>).';

create domain SIXTEEN_CHARACTERS char(16)

default NULL;

comment on domain SIXTEEN_CHARACTERS

is 'Domain for sixteen-alphanumeric-long fields.';

create domain SIXTY_CHARACTERS char (60)

default NULL;

comment on domain SIXTY_CHARACTERS

is 'Domain for sixty-alphanumeric-long fields.';

create domain TEN_CHARACTERS char(10)

default NULL;

comment on domain TEN_CHARACTERS

is 'Domain for ten-alphanumeric-long fields.';

create domain TWENTY_CHARACTERS char (20)

default NULL;

comment on domain TWENTY_CHARACTERS

is 'Domain for twenty-alphanumeric-long fields.';

create domain UNITS char(5)

default NULL;

comment on domain UNITS

is 'Domain for five-alphanumeric-long units.';

! Create tables.

create table SAMPLE_INFORMATION i

SAMPLE_TRACKING_ID

DATE_SAMPLE_ENTERED

DATE_ALL_WORK_COMPLETED

CUSTOMER SAMPLE ID

RESUITS_NEEDED_BY_DATE

CUSTOMER_PROJECT_NAME

WORK_CHARGE_NUMBER

CUSTOMER_SAMPLE_NAME

SAMPLE_TYPE

SAMPLE_SIZE

IDENTIFICATION_CODES

INTEGER_DATE_COUNT,

INTEGER DATE_COUNT,

IDENTIFICATION_CODES,

INTEGER_DATE_COUNT,

FORTY_CHARACTERS,

CHARGE_NUMBERS,

SIXTY_CHARACTERS,

TEN_CHARACTERS,

REAL_NUMBERS,

not null, 


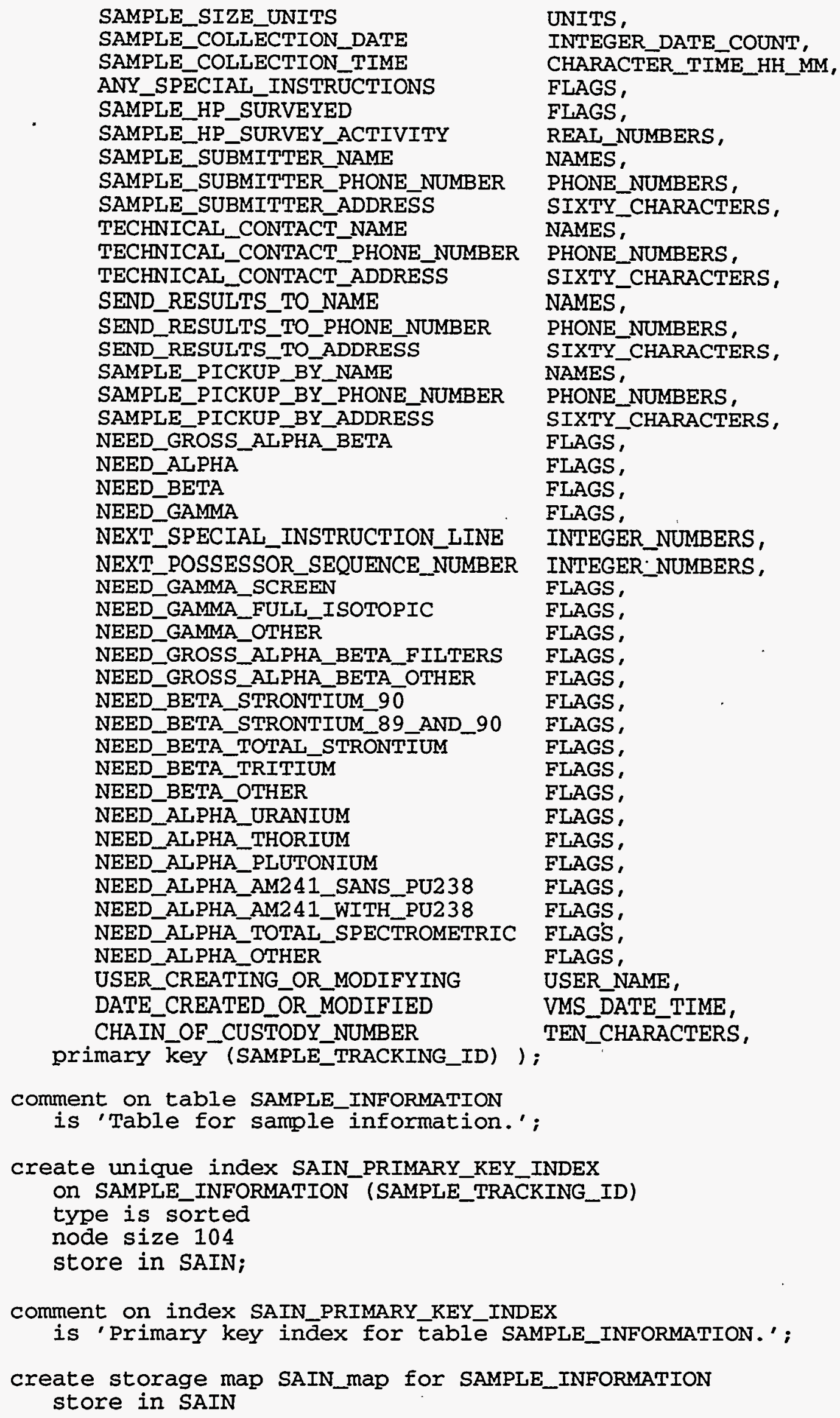


placement via index SAIN_PRIMARY_KEY_INDEX

disable compression;

create table ALPHA_URANIUM (

SAMPLE_TRACKING_ID

RESULTS_NEEDED_BY_DATE

DATE_ALI_WORK_COMPLETED

RESULTS_REPORT_CITATION

ANY_SPECIAL_INSTTRUCTIONS

USER_CREATING_OR_MODIFYING

DATE_CREATED_OR_MODIFIED

primary key (SAMPLE_TRACKING_ID),

foreign key (SAMPLE_TRACKING_ID)

IDENTIFICATION_CODES not null, references SAMPLE_INFORMATION (SAMPLE_TRACKING_ID) );

comment on table AIPHA_URANIUM

is 'Table for requested alpha analyses for Uranium radionuclides.';

create unique index ALUR_PRIMARY_KEY_INDEX

On ALPHA_URANIUM (SAMPLE_TRACKING_ID)

type is sorted

node size 104

store in ALUR;

comment on index ALUR_PRIMARY_KEY_INDEX

is 'Primary key index for table ALPHA_URANIUM.' ;

create storage map ALUR_map for ALPHA_URANIUM

store in ALUR

placement via index ALUR_PRIMARY_KEY_INDEX

disable compression;

create table AIPHA_THORIUM (

SAMPLE_TRACKING_ID

RESULTS_NEEDED_BY_DATE

DATE_ALE_WORK_COMPLETED

RESULTS_REPORT_CITATION

ANY_SPECIAL_INSTRUCTIONS

USER_CREATING_OR_MODIFYYING

DATE_CREATED_OR_MODIFIED

INTEER DATE COUNT,

DATE COUNT,

FLAGS,

USER_NAME,

VMS_DATE_TIME，

rimary key (SAMPLE_TRACKING_ID),

foreign key (SAMPLE_TRACKING_ID)

references SAMPLE_INFORMATION (SAMPLE_TRACKING_ID) );

comment on table ALPHA_THORIUM

is 'Table for requested alpha analyses for Thorium radionuclides.';

create unique index AITH_PRIMARY_KEY_INDEX

on ALPHA_THORIUM (SAMPLE_TRACKING_ID)

type is sorted

node size 104

store in ALTH;

comment on index ALTH_PRIMARY_KEY_INDEX

is 'Primary key index for table ALPHA_THORIUM.' ;

create storage map ALTH_map for AIPHA_THORIUM

store in ALTH

placement via index AITH_PRIMARY_KEY_INDEX

disable compression; 


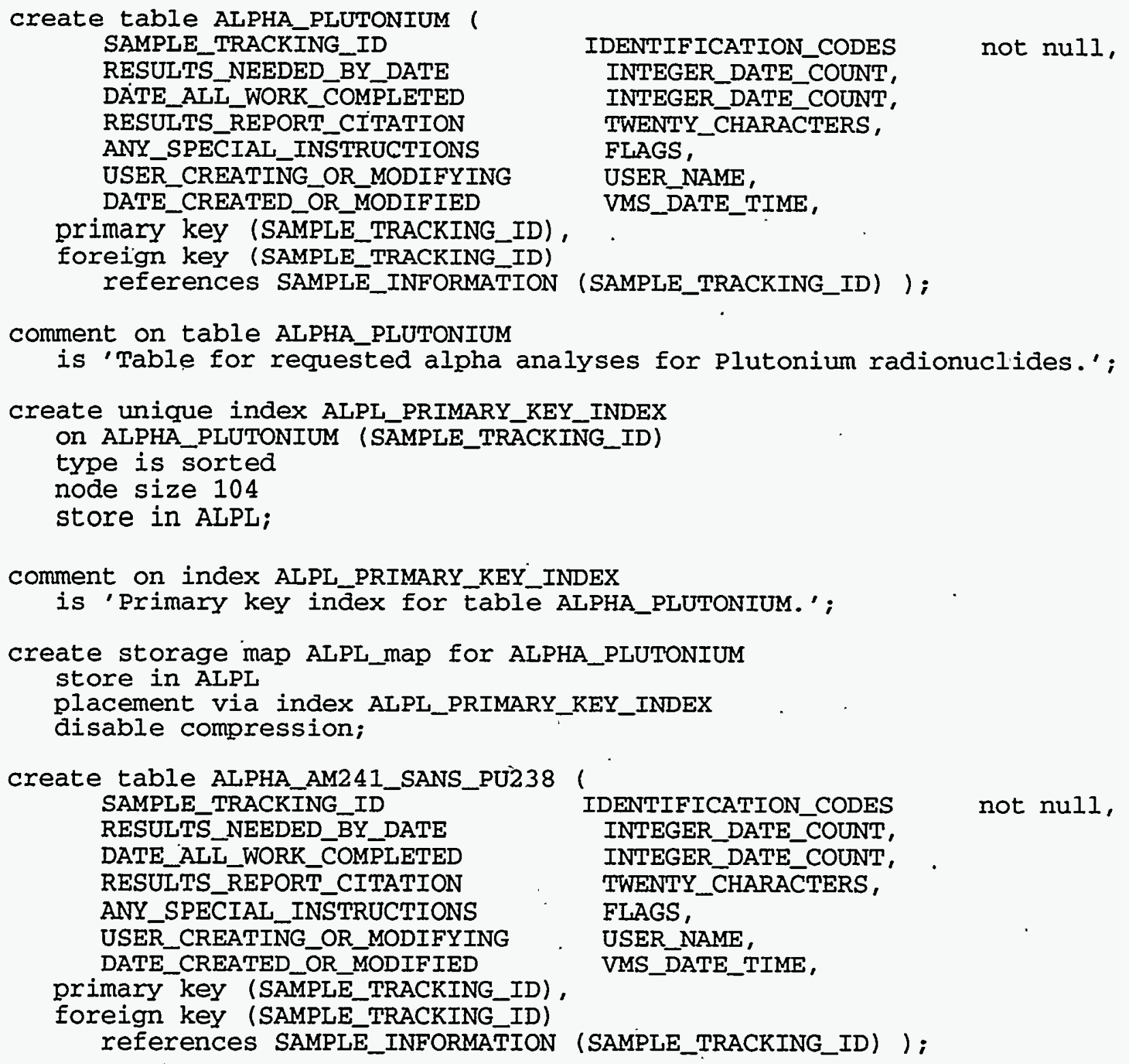


create table ALPHA_AM241_WITH_PU238（

SAMPLE_TRACKING_ID

RESULTS_NEEDED_BY_DATE

DATE_ALI_WORK_COMPLETED

RESULTS_REPORT_CITATION

ANY_SPECIAI_INSTRUCTIONS

USER_CREATING_OR_MODIFYING

DATE_CREATED_OR_MODIFIED

primary key (SAMPLE_TRACKING_ID),

foreign key (SAMPLE_TRACKING_ID)

references SAMPLE_INFORMATION (SAMPLE_TRACKING_ID) );
IDENTIFICATION_CODES not null,

INTEGER_DATE_COUNT,

INTEGER_DATE_COUNT,

TWENTY_CHARACTERS,

FLAGS,

USER_NAME,

VMS_DATE_TIME,

comment on table AIPHA_AM241_WITH_PU238

is 'Table for requested alpha analyses for Americium-241 combined with Plutonium-238.';

create unique index ALAMWIPU_PRIMARY_KEY_INDEX

on ALPHA_AM241_WITH_PU238 (SAMPLE_TRACKING_ID)

type is sorted

node size 104

store in AIAMWIPU;

comment on index.AI_AMWIPU_PRIMARY_KEY_INDEX

is 'Primary key index for table ALPHA_AM241_WITH_PU238.';

create storage map ALAMWIPU_map for ALPHA_AM241_WITH_PU238

store in ALAMWIPU

placement via index ALAMWIPU_PRIMARY_KEY_INDEX

disable compression;

create table ALPHA_TOTAL_SPECTROMETRIC (

SAMPLE TRACKING ID

RESULTS_NEEDED_BY_DATE

DATE_ALI_WORK_COMPLETED

RESULTS_REPORT_CITATION

ANY SPECIAI INSTRUCTIONS

USER_CREATING_OR_MCE_FYING

DATE_CREATED_OR_MODIZIED

IDENTIFICATION_CODES

INTEGER_DATE_COUNT,

INTEGER_DATE_COUNT,

TWENTY_CHARACTERS,

FLAGS,

USER_NAME,

VMS_DATE_TIME,

primary key (SAMPLE_TRACKING_ID),

foreign key (SAMPLE_TRACKING_ID)

references SAMPLE_INFORMATION (SAMPLE_TRACKING_ID) );

comment on table ALPHA_TOTAL_SPECTROMETRIC

is 'Table for requested total spectrometric alpha analyses.';

create unique index ALTOSP_PRIMARY_KEY_INDEX

On ALPHA_TOTAL_SPECTROMETRIC (SAMPLE_TRACKING_ID)

type is sorted

node size 104

store in ALTOSP;

comment on index ALTOSP_PRIMARY_KEY_INDEX

is 'Primary key index for table ALPHA_TOTAL_SPECTROMETRIC.';

create storage map ALTOSP_map for ALPHA_TOTAL_SPECTROMETRIC

store in ALTOSP

placement via index ALTOSP_PRIMARY_KEY_INDEX

disable compression; .

create table ALPHA_OTHER ( 
SAMPLE_TRACKING_ID . RESULTS_NEEDED_BY_DATE DATE_AII_WORK_COMPLETED RESULTS_REPORT_CITATION ANY_SPECIAL_INSTTRUCTIONS USER_CREATING_OR_MODIFYING DATE_CREATED_OR_MODIFIED primary key (SAMPLE_TRACKING_ID), foreign key (SAMPLE_TRACKING_ID) references SAMPLE_INFORMATION (SAMPLE_TRACKING_ID) );
IDENTIFICATION_CODES INTEGER_DATE_COUNT, INTEGER_DATE_COUNT, TWENTY_CHARACTERS, FIAAGS, USER_NAME, VMS_DATE_TIME,

comment on table ALPHA_OTHER

is "Table for requested alpha analyses for other, unlisted radionuclides.';

create unique index ALOT_PRIMARY_KEY_INDEX

On ALPHA_OTHER (SAMPLE_TRACKING_ID)

type is sorted

node size 104

store in ALOT;

comment on index ALOT_PRIMARY_KEY_INDEX

is 'Primary key index for table ALPHA_OTHER.';

create storage map ALO'T_map for ALPHA_OTHER

store in ALOT

placement via index ALOT_PRIMARY_KEY_INDEX

disable compression;

create table BETA_STRONTIUM_90 (

SAMPLE_TRACKING_ID

DETECTOR_COUNT LENGTH

DETECTOR_COUNT_LENGTH_UNITS

RESULTS_NNEEDED_BY_DATE

RESULTS_NEEDED_BY_TIME

DATE_ALI_WORK_COMPLETED

RESULTS_REPORT_CITATION

ANY_SPECIAL_INSTRUCTIONS

USER_CREATING_OR_MODIFYING

DATE_CREATED_OR_MODIFIED

primary key (SAMPLE_TRACKING_ID),

foreign key (SAMPLE_TRACKING_ID)

references SAMPLE_INFORMATION (SAMPLE_TRACKING_ID) );

comment on table BETA_STRONTIUM_90

is 'Table for requested beta analyses for strontium-90.';

create unique index BEST90_PRIMARY_KEY_INDEX

On BETA_STRONTIUM_90 (SAMMLE_TRACKING_ID)

type is sorted

node size 104

store in BEST90;

comment on index BEST90_PRIMARY_KEY_INDEX

is 'Primary key index for tab̆le BETA_STRONTIUM_90.';

create storage map BEST90_map for BETA_STRONTIUM_90

store in BEST90

placement via index BEST90_PRIMARY_KEY_INDEX

disable compression; CHARACTER_TIME_HH_MM, INTEGER_DATE_COUNT, IENTY CHARACTERS, USER NAME, VMS_DATE_TIME, not null,

$$
\text { IDENTIFICATION_CODES not null, }
$$




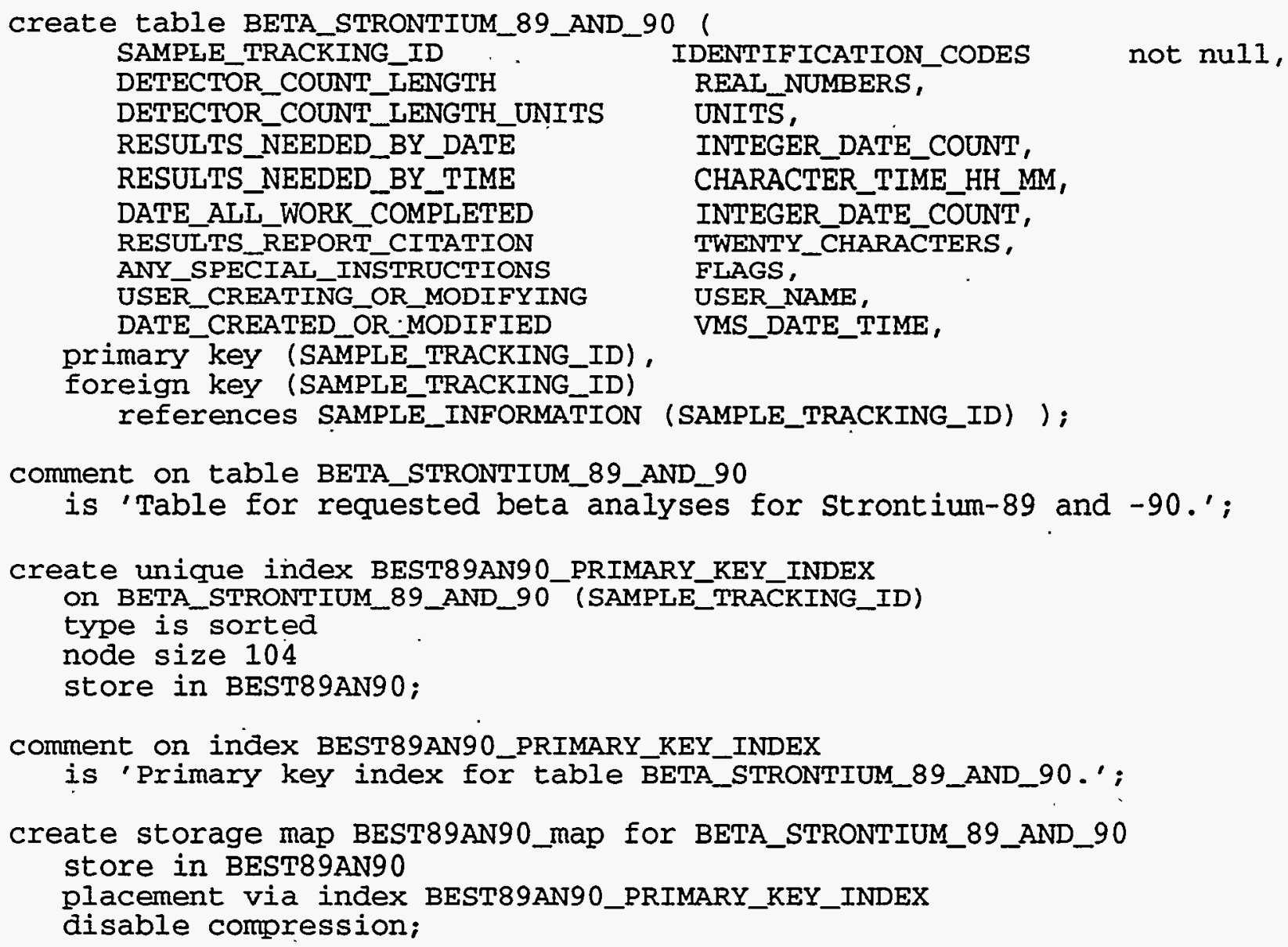

comment on table BETA_TOTAI_STRONTIUM

is 'Table for requested beta analyses for total strontium.';

create unique index BETOST_PRIMARY_KEY_INDEX

On BETA_TOTAL_STRONTIUM (SAMPLE_TRACKING_ID)

type is sorted

node size 104

store in BETOST;

comment on index BETOST_PRIMARY_KEY_INDEX

is 'Primary key index for table BETA_TOTAL_STRONTIUM.' ; . 


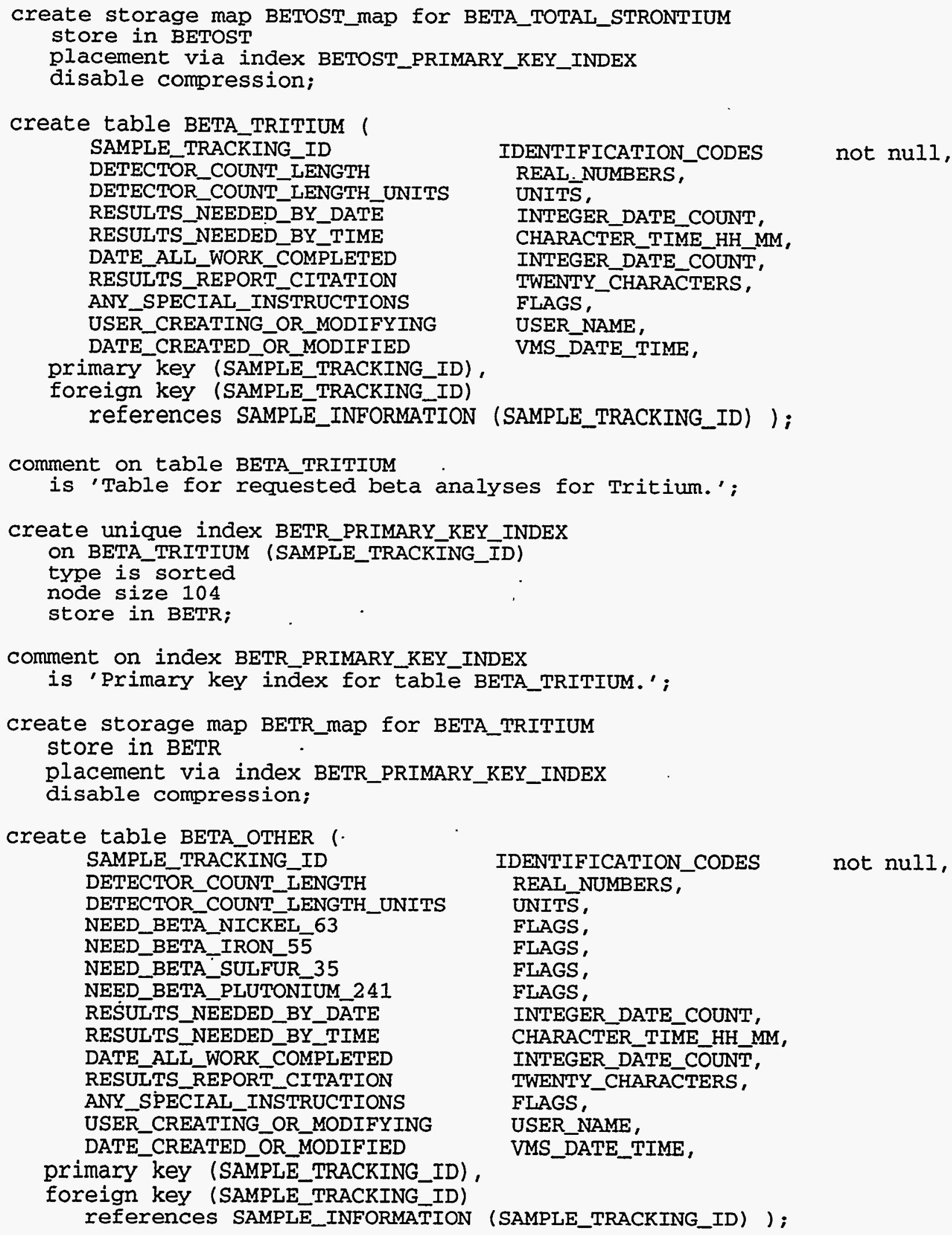

is 'Table for requested beta analyses for other, unlisted radionuclides.'; 


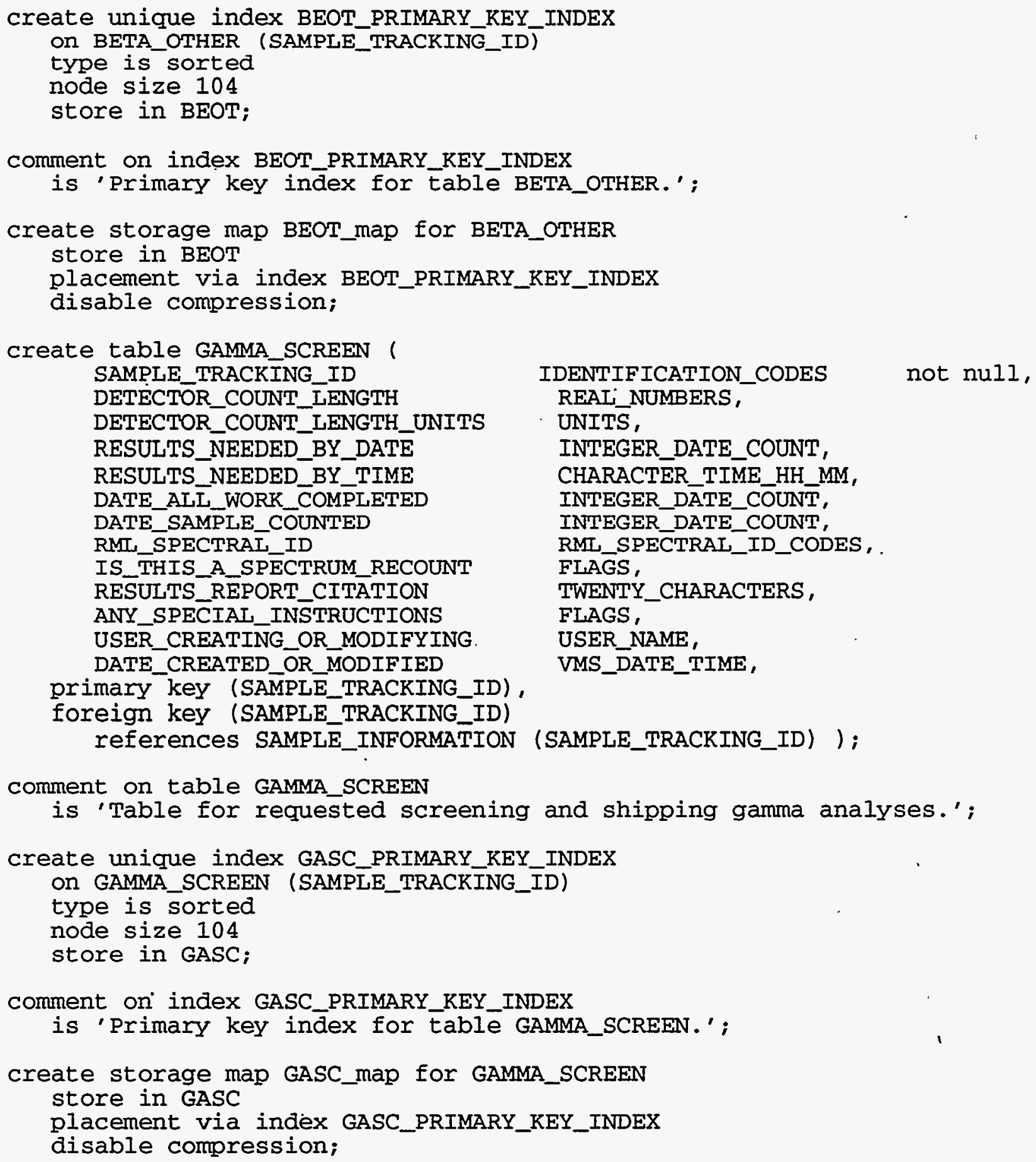


ANY_SPECIAL_INSTRUCTIONS . USER_CREATING_OR_MODIFYING DATE_CREATED_OR_MODIFIED

primary key (SAMPLE_TRACKING_ID), foreign key (SAMPLE_TRACKING_ID) references SAMPLE_INFORMATION (SAMPLE_TRACKING_ID) );
FLAGS, USER NAME, VMS_DATE_TIME,

comment on table GAMMA_FUIL_ISOTOPIC

is 'Table for requested full isotopic gamma analyses.';

create unique index GAFUIS_PRIMARY_KEY_INDEX

On GAMMA_FULI_ISOTOPIC (SAMPLE_TRACKING_ID)

type is sorted

node size 104

store in GAFUIS;

comment on index GAFUIS_PRIMARY_KEY_INDEX

is 'Primary key index for table GAMMA_FULL_ISOTOPIC.';

create storage map GAFUIS_map for GAMMA_FULI_ISOTOPIC

store in GAFUIS

placement via index GAFUIS_PRIMARY_KEY_INDEX

disable compression;

create table GAMMA_OTHER ( SAMPLE_TRACKING_ID

DETECTOR_COUNT_LENGTH

DETECTOR_COUNT_LENGTH_UNITS

RESULTS_NNEEDED_BY_DATE

RESULTS_NEEDED_BY_TIME

DATE_ALI_WORK_COMPLETED

DATE_SAMPLE_COUNTED

RMI_SPECTRA $\bar{L}$ ID

IS_THIS_A_SPECTRUM_RECOUNT

RESULTS_REPORT_CITATION

ANY_SPECIAI_INSTRUCTIONS

USER_CREATINTG_OR_MODIFYING

DATE_CREATED_OR_MODIFIED

primary key (SAMPLE_TRACKING_ID)

foreign key (SAMPLE_TRACKING_ID)

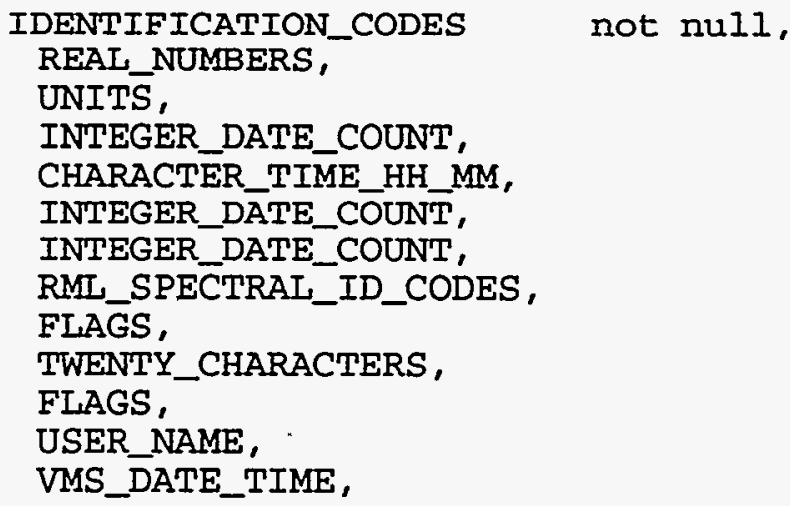




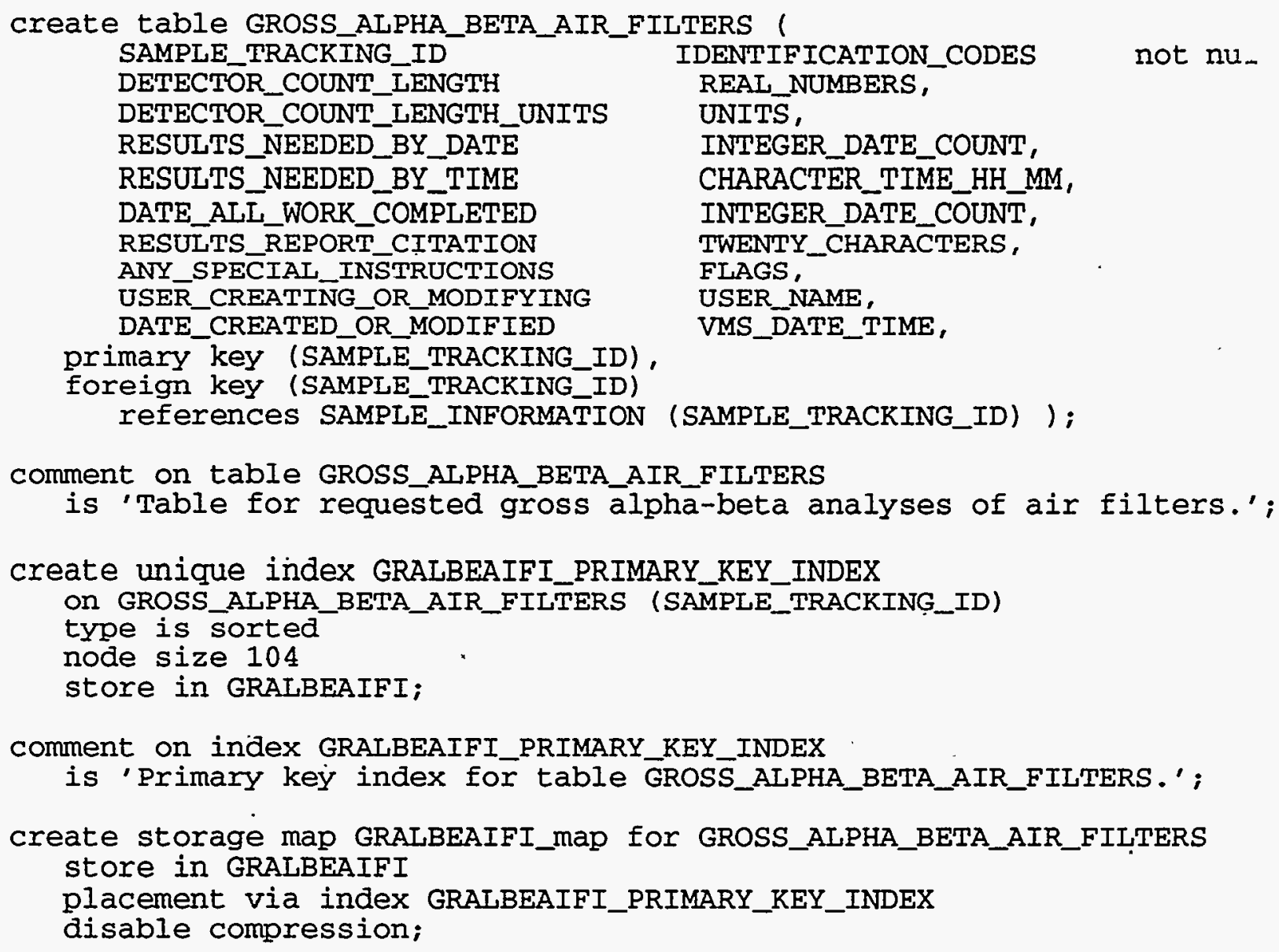

comment on table GROSS_ALPHA_BETA_OTHER

is 'Table for requested gross alpha-beta analyses of other, unlisted samples.';

create unique index GRALBEOT_PRIMARY_KEY_INDEX

On GROSS_ALPHA_BETA_OTHER (SAMPLE_TRACKING_ID)

type is sorted

node size 104

store in GRALBEOT;

comment on index GRALBEOT_PRIMARY_KEY_INDEX

is 'Primary key index for table GROSS_ALPHA_BETA_OTHER.' ; 


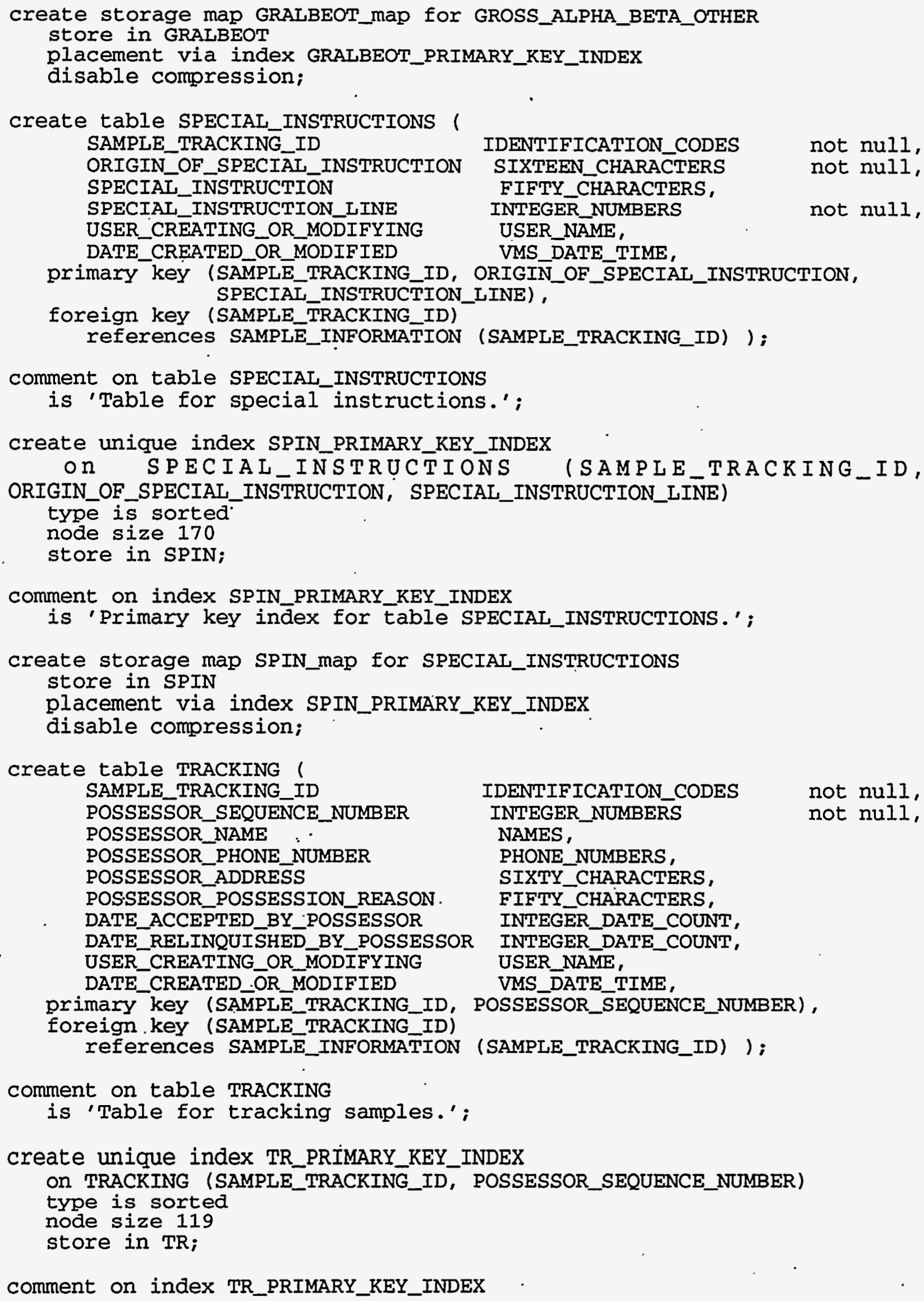

not null, not null,

not null, 
is 'Primary key index for table TRACKING.';

create storage map TR_map Eor TRACKING

store in TR

placement via index TR_PRIMARY_KEY_INDEX

disable compression;

commit;

exit; 


\section{B. LOTUS® 1-2-3® PRINT FILES CONTAINING THE ENTITY- AND SCREEN-SORTED ATTRIBUTE DESCRIPTION LISTS}

The series of blank lines which appear regularly throughout these two files are a result of these being Lotus@ 1-2-3® print files; the series of blank lines correspond to page throws. The tool CREATE_SCHEMA ignores these page throws.

\section{B.1 Entity-Sorted List}

Rdb Entity-Sorted Worksheet - duplicate entries retained for cursor generation - dashes demarcate entities.

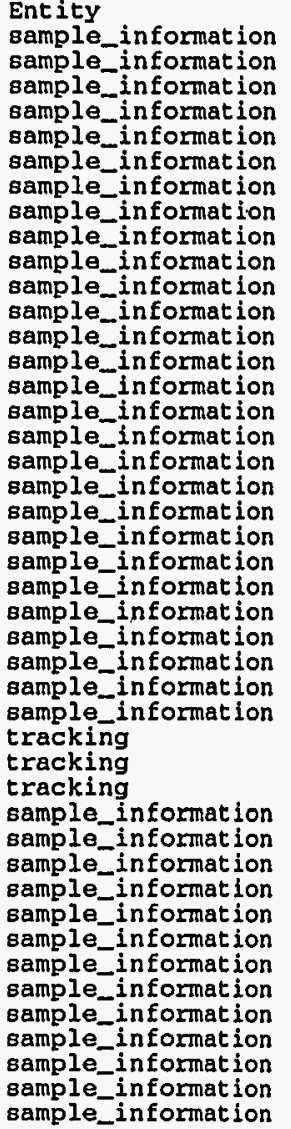

Attribute

. date_sample_entered

date_all_work_completed

.

customer_projeby_date

. work charge number

.customer_sample_name

- sample_type

- Bample_size_units

- sample_collection_date

- sample_collection_time

- any_special_instructions

- sample_hp_survey_act ivity

- sample_submitter_name

. sample_submitter_phone_number

- sample_submitter_addres

-technical_contact_name

technical_contact_addres

send_resuits_to_name

作

- sample pickup by name

- sample_pickup_by_phone_number

. sample_pickup_by_address

-possessor_name

- possessor_phone_number

- possessor_address

-chain_of_custody_number

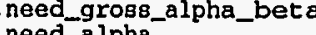

- need_alpha

need_gamma

next_special_instruction_line

next_possessor_sequence_number

- need_gamma_screen

- need_gamma_full_isotopic

- need_gamma_other

need_gross_alpha_beta_filters

- need_gross_alpha_beta_other

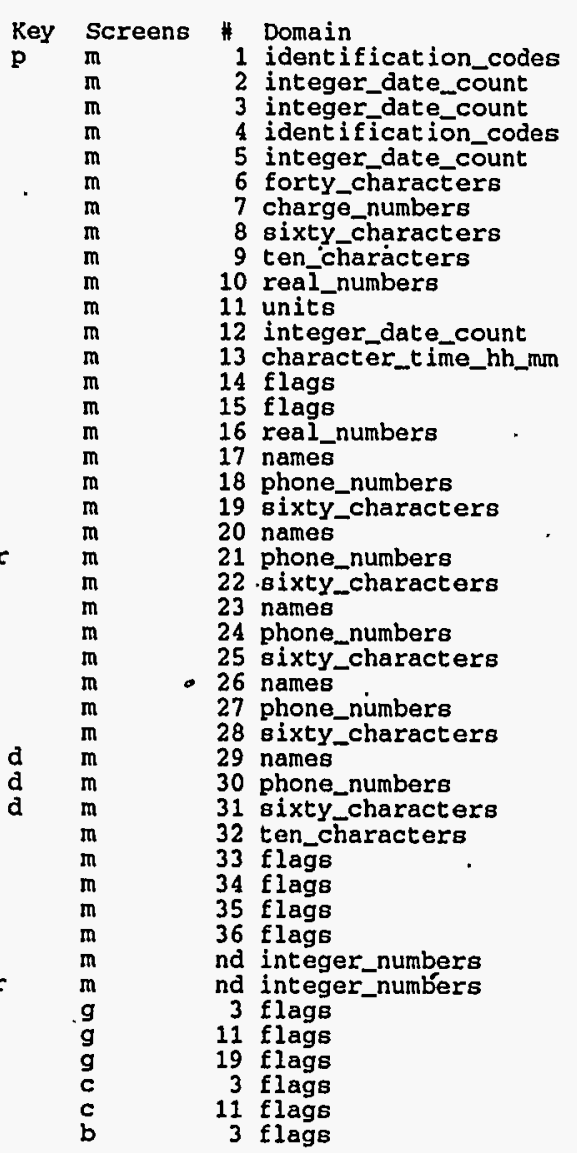

Domain .

Domain

character_time_hh_mm

charge_numbers

flags - haracters

forty_characters

identification_codes

integer_date_count

integer_numbers

names

phone_numbers

real_numbers

mi_spectral_id_codes

sixty characters

ten_characters

twenty characters

units 
sample_information sample_in formation sample_in formation sample information sample_information sample information sample information sample in formation sample_information sample_information alpha_uranium need_beta_strontium_89_and_90 . - need-beta-tritium - need_beta_other

need alpha thorium

need alpha plutonium

need alpha am 41 sans pu238

need_alpha_am241_with pu238

need_alpha_total_spectrometric need_alpha_other

. sample_tracking_id sample_information

alpha_uranium

alpha_uranium

alpha_uranium

alpha_uranium

alpha_thorium

ample_information

alpha_thorium

alpha_thorium

alpha_thorium

alpha_pluton ium

ample information

alpha_plutonium

alpha_plutonium

alpha_plutonium

alpha_am241_sans_pu238

sample_in formation

alpha_am241_sans_pu238

alpha_an241_sans_pu238

alpha_am241_oan_pu238

alpha_am241 with pu23

sample_in formation

alpha_am241_with_pu23

alpha_am241_with_pu238

alpha_am241_with_pu23

alpha_am241_with_pu238

alpha_total_spectrometric

sample_information

alpha_total_spectrometric

alpha_total_spectrometric

alpha_total_spectrometric

alpha_other

sample_information

alpha_other

alpha_other

alpha_other .customer_sample_name results_needed_by_date date_al__work_completed results_report_citation . any_special_instructions

. sample_tracking_id stomer_sample_name results_needed_by_date results_report_citatio any_special_instructions

. sample_tracking_id

customer_sample_name date aly nork_by_date results report citation any_special instruction

.sample_tracking_id . customer_sanple_name results_needed_by_date _date_all_work_completed any_special_instructions

sample_tracking_id

customer_sample_name

results_needed_by_date date_ali_work_completed any_special_instructions

\section{sample_tracking_id}

customer_sample_name results_needed_by_date date_ali__work_completed results_report citation

\section{. sample_tracking_id}

customer_sample_name

results_needed_by_date

re

any special instructions
2 sixty_characters

integer_date_count

integer_date_count

twenty_characters

1 identification codes

sixty_characters

integer_date_coun

11 twenty_characters

12 flags

1 identification_codes

2 sixty_characters

15 integer date count

16 twenty characters

17 flags

1 identification_codes

2 sixty_characters

19 integer_date_count

21 integer date count

f2 1 lage_characters

1 identification_codes

2 sixty_characters

24 integer_date_coun

25 integer_date count

26 twenty_characters

\section{7 flags}

1 identification codes

2 sixty_characters

integer_date_coun

31 twenty_characters

32 flags

1 identification_codes

2 sixty_characters

35 integer_date_count

36 twenty characters

37 flags 


$\begin{array}{ll}\text { beta_strontium_90 } & \text {-sample_tracking_id } \\ \text { sample_information } & \text {-customer_sample_name } \\ \text { beta_strontium_90 } & \text {-detector_count_length } \\ \text { beta_stront_ium_90 } & \text {-detector_count_length_units } \\ \text { beta_strontium_90 } & \text {-results_needed_by_date } \\ \text { beta_strontium_90 } & \text {-results_needed_by_time } \\ \text { beta_strontium_90 } & \text {-date_ali_work_completed } \\ \text { beta_strontium_90 } & \text {-results_report_citation } \\ \text { beta_strontium_90 } & \text {.any_special_instructions } \\ \text { beta_strontium_89_and_90 } & \text {.sample_tracking_id } \\ \text { sample_information } & \text {.customer_sample_name }\end{array}$
$\mathrm{f} / \mathrm{p} \quad \mathrm{b} \quad I$ identification_codes 2 sixty_characters
4 real_number
5 units
6 integer_date_count
character_time_hh_m
9 twenty_characters
10 flags
$\begin{array}{ll}\text { f/p } & \text { b } \\ \text { b } & \frac{1}{2} \text { identification_codes } \\ \text { sixty_characters }\end{array}$

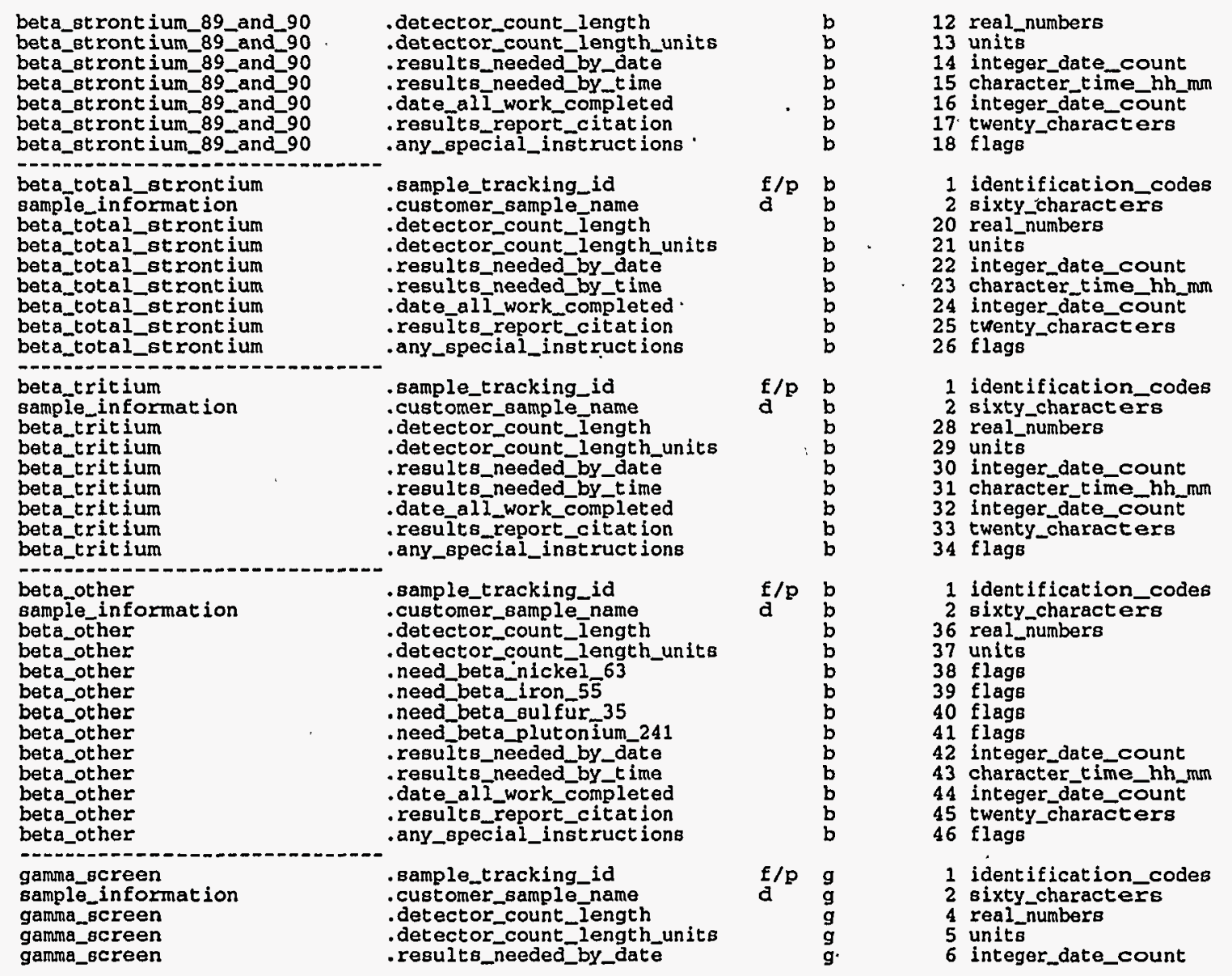


gamna_screen

gamma_Bcreen

gamma_Bcreen

gen

gamma screen

gamma_Bcreen

gamma_full_isotopic

sample_information

gamma_ful1_isotopic

gamma-cull-isotopic

gamma ful isotopic

gamma_fuli isotopic

gamma_full_isotopic

gamma_full_isotopic

gamma_ful__isotopic

gamma_full_isotopic results_needed_by_time date_al__work_completed date sample_count is_this_a_spectrum_recoun results report citation any_special_instructions

\section{sample_tracking_id}

customer_sample name

detector_count_length

detector_count_length_unit

result_ needed_by_date

date al $\overline{1}$ work completed

date sample counted

mi_spectral_id

.is_this_a_spectrum_recount

-results_report_citation
7 character_time_hh_mm

integer_date_count

10 inl spectral id codes

11 Elags

12 twenty_characters

1 identification_codes

2 sixty_characters

real_numbers

16 units

17 integer_date_count

19 integer date count

20 integer date count

21 rml_spectral_id_codes

22 flags

23 twenty_characters gamma_ful1_isotopic

\section{gamma_other \\ ample_information}

gamma_other

gamma_other

gamma other

gamma other

gemsis other

gami:

gam

gath:

gamirict.

gross_alpha_beta_air_filters

gross al

gross al

gross_alpha beta_air filters

gross_alpha_beta_air_filters

gross_alpha_beta_air_filters

gross_alpha_beta_air_filters

gross_alpha_beta_other

grose_alpha_beta_oth

gross_alpha_beta_othe

gross_alpha_beta_other

gross_alpha_beta_other

gross_alpha_beta_other

gross_alpha_beta_other

gross_alpha_bata_other

grose_alpha_beta_other

special_instruction

sample in formation

special inst ruction

special inst ruction

special_instruction any_special_instructions

sample_tracking_id

customer_sample_name

_detector_count_lengt

detector_count_length_units

resulter couned leygen un

date ali work completed

date sample counted

nil_spectral_id

is_this_a_spectrum_recount

results_report_citation

sample_tracking_id

\section{ustomer_sample_name}

detector count length unit

esults_needed_by_date

(1)

results_report_citation

any_special_instruct ions

sample_tracking_id

customer_sample_name

detector_count length unit

results_needed_by_date

results needed_by_time

.date_al1_work_completed results_repor_citation

sample_tracking_id

customer sample name origin_of _special_in

special_instruction_line

$c$
$c$
$c$
$c$
$c$
$c$
$c$
$c$
$c$
$c$
$c$
$c$
$c$
$c$
$c$
$c$
$c$
$c$
$c$
$c$
$i$
$i$
$i$
$i$
$i$
$i$

24 flags

ident ification_codes

2 sixty_character

27 un'tis

28 integer_date_count

29 character_time_hh_mm

31 integer date count

$32 \mathrm{rml}$ spectral_id_codes

33 flags

34 twenty_characters

1 identification_codes

2 sixty_character

5 units

7 integer_date_count

7 character_time_hh_mm

8 integer_date_count

1 identification_codes

2 sixty_characters

12 real_numbers

13 units

14 integer_date_count

15 character_time_hh_mm

16 integer_date_count

twenty_chara

1 identification_codes

sixty_characters

3 sixteen_characters

nd integer_numbers 
tracking

sample_information

trackin

tracking

tracking

tracking

tracking
- sample_tracking_id

- custossor name name

- possessor_phone_number

- possessor_adares

- possessor_possession_reason

-date_accepted_by_possessor

inguished_by_posseseor

- possessor sequence number $f / p 1 t \quad 1$ identification_codes

2 sixty_characters

names

phone_numbers

sixty_characters

fifty_characters

integer_date_count

8 integer_date_count 


\section{B.2 Screen-Sorted List}

Worksheet for Sorting Entities and Attributes and Assigning Keys and Relationships.

Base Worksheet - Sorted by Screen

Entity

sample_information sample_information sample_information sample_information sample_information sample information sample information sample_information sample_information sample information sample_information sample_information sample_information sample information sample_information sample_information sample_information sample_information sample_information sample_in formation sample information sample informat ion sample_information sample_information sample_information sample_information sample_information sample information tracking

tracking

tracking

sample_information sample_information sample_information sample_information sample_information sample_information sample_information

alpha_uranium sample_information sample_information alpha_uranium alpha_uranium alpha uranium alpha uranium sample_information alpha_thorium. alpha_thorium alpha_thorium alpha_thorium sample_information alpha_plutonium alpha plutonium alpha_plutonium alpha_plutonium
Attribute

-sample_tracking_id

- date_sample_entered

-date_al1_work_completed

- customer_sample_id

-results_needed_by_date

- customer project ñame

-work charge number

.customer_sample_name

- sample_type

sample_size

- sample_size_units

-sample_collection_date

sample collection time

. any_special_instructions

-sample_hp_surveyed

-sanple_hp_survey_activity

- sample_subratter_name

-sample_submitter_phone_number

-sample_submitter_address

-technical contact name

.technical_contact_phone_number

technical contact address

- send_resuíts_to_name

. send_results_to_phone_number

- send_results_to_address

. sample_pickup_by_name

- sample pickup by phone number

. sample_pickup_by_address

possessor name

- possessor_phone_number

- possessor_address

-chain_of_custody_number

-need_gross_alpha_beta

- need_alpha

- need_beta

- need_gamma

- next_possessor_sequence_number

-next_special_instruction_line

.sample_tracking_id

. customer sample name

- need alpha uranium

-resuitts_needed_by_date

.date ali _work_completed

-results_report citation

. any_special_instructions

need alpha thorium

resuitss_needed_by_date

. date all work completed

-results_report_citation

. any_special_instructions

- need_alpha_plutonium

-results_needed_by_date

- date ali work completed

-results_report_citation

-any_special_instructions
Key Screens \# Domain

p m 1 identification_codes

2 integer_date_count

3 integer_date_count

4 identification_codes

5 integer_date_count

6 forty_characters

7 charge numbers

8 sixty characters

9 ten characters

10 real_numbers

11 units

12 integer_date_count

13 character_time_hh_mm

14 flags

15 flags

16 real_numbers

17 names

18 phone_numbers

19 sixty_characters

20 names

21 phone numbers

22 sixty_characters

23 names

24 phone_numbers

25 sixty_characters

26 names

27 phone_numbers

28 sixty_characters

29 names

30 phone_numbers

31 sixty_characters

32 ten_characters

33 flags

34 flags

35 flags

36 flags

nd integer_numbers

nd integer_numbers

1 identification codes

2 sixty_characters

3 flags

4 integer_date_count

5 integer_date_count

6 twenty_characters

7 flags

$8 \mathrm{flags}$

9 integer_date_count

10 integer_date_count

11 twenty

12 flags

14 integer date count

15 integer date count

16 twenty characters

17 flags sample_information alpha_am241_sans_pu238 alpha_am241_sans_pu238 alpha_am241_sans_pu238 alpha_an241_sans_pu238 sample information alpha an241 with pu238 alpha_am241_with_pu238 alpha_am241_with_pu238 alpha_am241_with_pu238 sample_information alpha_total_spectrometric alpha_total_spectrometric alpha_total_spectrometric .need_alpha_am241_sans_pu238

-results_needed_by_date

.date all_work_completed

- results report citation

- any special instructions

. need_alpha_am241_with_pu238

- results_needed_by_date

. date alI work_completed

- results report citation

any special instructions

- need_alpha_total_spectrometric

- resuits_needed_by_date

-date_alI_work_completed

- results report citation
18 flags

19 integer_date_count

20 integer date_count

21 twenty characters

22 flags

23 flags

24 integer_date_count

25 integer_date_count

26 twenty_characters

27 flags

28 flags

29 integer_date_count

30 integer_date_count

31 twenty_characters 
alpha_total_spectrometric gample_information alpha_other alpha_other alpha other alpha_other

beta_strontium 90 sample_information sample information bete_strontium_90 beta_etrontium_90 beta_st ront ium_90 beta_strontium 90 beta strontium 90 beta stront jum 90 beta etront ium-90 sample_information beta_strontium_89_and_90 beta_strontium_89_and_90 beta_strontium_89_and_90 beta strontium 89 and 90 beta_strontium_89_and_90 beta strontium 89 and 90 beta_strontium_89_and_90 sample_information beta_total_strontium beta total stront ium beta_total_strontium beta total strontium beta total strontium beta_total strontium beta total strontium sample information beta_tritium beta_tritium beta_tritium beta_tritium beta_tritium beta_tritium sampIe_information beta other beta-other beta_other beta_other
- any_special_instructions - need_alpha_other

-results_needed_by_date

-date_all_work_completed

results report citation

.any_special_instructions

-sample_tracking_id

- customer sample name

- need_beta__strontium_90

.detector_count_length

-detector_count_length_units

-results_needed_by_date

-results_needed_by_time

-date_all_work_completed

-results_report_citation

- any_special_instructions

- need_beta_strontium_89_and_90

-detector_count_length

-detector_count_length_units

-results_needed_by_date

- results needed by time

.date_ali_work_completed

-results_report_citation

. any_special_instructions

- need_beta_total_strontium

-detector_count_length

-detector_count_length_units

-results_needed_by_date

- results needed by time

date_ali__work_completed

-results_report_citation

-any_special_instructions

- need_beta_tritium

- detector_count_length

detector count length units

-results_needed_by_date

-results_needed_by_time

date_all_work_completed

- resuIts_report_citation

- need_bera_other

- detector_count_length

detector count length units

need beta nickel 63

- need_beta_iron_55
32 flags

33 flags

34 integer_date_count

35 integer date_count

36 twenty_characters

37 flags

1 identification_codes

2 sixty characters

3 flags

4 real_numbers

5 units

6 integer_date_count

7 character_time_hh_mm

8 integer_date_count

9 twenty_characters

10 flags

11 flags

12 real_numbers

13 units

14 integer date_count

15 character_time_hh_mm

16 integer date count

17 twenty_characters

18 flags

19 flags

20 reaI_numbers

21 units

22 integer_date_count

23 character_time_hh mm

24 integer_date_count

25 twenty_characters

26 flags

27 flags

28 real_numbers

29 units

30 integer_date_count

31 character_time_hh_mm

32 integer_date_count

33 twenty_characters

34 flags

36 real_numbers

37 units

38 fiag

38 flags
39 flags beta_other

beta_other

beta_other

beta_other

beta_other

beta_other

beta_other

gainna_screen

sample information

sample information

gamma_screen

gamma_screen

gamma_screen

gamma_screen

gamma_Bcreen

gamma screen

gamma screen

gamma_screen

gamna_screen

gamna_screen

sample_information

gamma_full_isotopic

gamma full isotopic

gamma full isotopic

gamma full isotopic

gamma_full_isotopic

gamma_full_isotopic

gamma_full_isotopic

gamma_full_iвotopic

ganma_full_isotopic
- need_beta_sulfur_35

- need_beta_plutonium_241

- results needed by date

results_needed_by_time

- date_ali_work_completed

-resuIts_report_citation

-any_special_instructions

-sample_tracking_id

.customer_sample_name

need gamna screen

-detector_count_length

.detector_count_length_units

-results_needed_by_date

results needed_by_time

- date all_work completed

date sample counted

- rml_spectral_id

is this a spectrum recount

results_report_citation

.any_special_instructions

- need_gama_full_isotopic

- detector_count_length

-detector_count_length_units

results_needed_by_date

results_needed_by_time

.date_ali_work_completed

date_sample_counted

- rmi_spectral_id

is_this_a spectrum recount

results_report_citation
40 flags

42 integer date count

43 character_time_hh_mm

44 integer_date_count

45 twenty_characters

46 flags

1 identification_codes

2 sixty_characters

3 flags

4 real_numbers

5 units

6 integer_date_count

7 character_time_hh_mm

8 integer_date_count

9 integer date count

$10 \mathrm{rml}$ spectral_id_codes

11 flags

12 twenty_characters

13 flags

14 flags

15 reaI_numbers

16 units

17 integer_date_count

18 character_time_hh_mm

19 integer_date_count

20 integer_date_count

$21 \mathrm{rml}$ spectral_id_codes

22 flags

23 twenty_characters gamma_full_isotopic sample_information any special instructions

-need_gamma_other
24 flags

25 flags 
gamma_other

gamma_other

gamma_other

gamma other

gamma_other

gamma_other

gamma_other

gamma_other

gamma_other

gamna_other

gross_alpha_beta_air_filters sample_information sample information

gross_alpha_beta_air_filter

gross_alpha beta_air filters

gross \&lpha beta air filters

gross_alpha_beta_air_filters

gross alpha beta-air filters

gross_alpha beta_air_filter

gross_alpha_beta_air_filters

sample information

gross_alpha_beta_other

gross_alpha_beta_other

gross alpha beta other

gross alpha_beta_other

gross_alpha_beta_other

gross_alpha_beta_other

gross_alpha_beta_other

special instructions sample information speciaI instructions special_instructions special-instructions

tracking

sample information

tracking

tracking

tracking

tracking

tracking

tracking

tracking .detector_count_length

. detector_count_length_units

- results_needed_by_date

- results needed by time

.date_ali_work_completed

- date_sample_counted

.rml_spectral_id

- is_this_a_spectrum_recount

-results_report_citation

-any_special_instructions

.sample_tracking_id

customer_sample_name

. detector_count_length

detector_count_length_units

-results_needed_by_date

results needed by t ime

date all work completed

date_all_work_completed

. any_special_instructions

- need_gross_alpha_beta_other

-detector_count_length

detector count length_units

-results_needed_by_date

-results needed by time

.date_ali_work_completed

-results_report_citation
26 real_numbers

27 units

28 integer_date_count

29 character time_hh_mm

30 integer_date_count

31 integer_date_count

32 rmI_spectral_id_codes

33 flags

34 twenty_characters

35 flags

1 identification_codes

2 sixty_characters

3 Elags

4 real_numbers

5 units

6 -integer_date_count

7 character_time_hh_mm

8 integer_date_count

9 twenty characters

10 flags

11 flags

12 real_numbers

13 units

14 integer date count

15 character time hh

16 integer date count

17 twenty_characters .ány_special_instructions

c

. sample_tracking_id

- customer sample name

$f / p 1 i$

-origin_of_special_instruction p

-special_instruction

-special_instruction_line

p3

- sample tracking id

.customer_sample_name.

f/pl t

-possessor_name

- possessor_phone_number

- possessor_address

- possessor possession reason

- date_accepted_by_possessor

-date_relinquished_by_possessor

-possessor_sequence_number d

t

18 flags

1 identification_codes

2 sixty characters

. 3 sixteen characters

4 fifty_characters

nd integer_numbers

1 identification_codes

2 sixty_characters

3 names

4 phone_numbers

5 sixty_characters

6 fifty characters

7 integer_date_count

8 integer_date_coun

nd integer_numbers 


\section{EXECUTION OF CREATE SCHEMA - A SAMPLE TERMINAL SESSION}

What the user enters appears in bold text in the following.

\section{\$ ecreate_schema}

Enter the name of the schema to be created: sample_tracking For domain CHARACTER_TIME_HH_MM:

Enter the data type for this domain (char/date/int/real): c Enter the length of the character domain: 5

Enter the default value (return says NULL):

Enter any desired comment: Domain for time in the form hh:mm. For domain CHARGE_NUMBERS:

Enter the data type for this domain (char/date/int/real): c

Enter the length of the character domain: 9

Enter the default value (return says NULL): PF7400000 numbers.

Enter any desired comment: Domain for nine-alphanumeric-long charge For domain FIFTY_CHARACTERS:

Enter the data type for this domain (char/date/int/real): c

Enter the length of the character domain: $\mathbf{5 0}$

Enter the default value (return says NULL):

Enter any desired comment: Domain for fifty-alphanumeric-long fields. For domain FLAGS:

Enter the data type for this domain (char/date/int/real): c

Enter the length of the character domain: 1

Enter the default value (return says NULL): $\mathbf{N}$ flags.

Enter any desired comment: Domain for one-character-long yes/no

For domain FORTY_CHARACTERS:

Enter the data type for this domain (char/date/int/real): c Enter the length of the character domain: $\mathbf{4 0}$

Enter the default value (return says NULI): 
Enter any desired comment: Domain for forty-alphanumeric-long fields. For domain IDENTIFICATION_CODES:

Enter the data type for this domain (char/date/int/real): c Enter the length of the character domain: 12

Enter the default value (return says NULI):

Enter any desired comment: Domain for twelve-character-Iong identification codes.

For domain INTEGGER_DATE_COUNT:

Enter the data type for this domain (char/date/int/real): $i$

Enter the default value (return says NULL):

Enter any desired comment: Domain for integer*4 date count starting at January 1, 1990.

For domain INTEGER_NUMBERS:

Enter the data type for this domain (char/date/int/real): i

Enter the default value (return says NULL):

Enter any desired comment: Domain for integer*4 numbers.

For domain NAMES:

Enter the data type for this domain (char/date/int/real): c

Enter the length of the character domain: 25

Enter the default value (return says NULL):

Enter any desired comment: Domain for twenty-five-alphanumeric-1ong names.

For domain PHONE_NUMBERS:

Enter the data type for this domain (char/date/int/real): c

Enter the length of the character domain: 12

Enter the default value (return says NULL):

Enter any desired comment: Domain for twelve-alphanumeric-long phone numbers.

For domain REAL_NUMBERS:

Enter the data type for this domain (char/date/int/real): $\boldsymbol{r}$

Enter the default value (return says NULL):

Enter any desired comment: Domain for real*4 numbers. 
For domain RML_SPECTRAL_ID_CODES:

Enter the data type for this domain (char/date/int/real): c

Enter the length of the character domain: 14

Enter the default value (return says NULL):

Enter any desired comment: Domain for fourteen-character-long RML spectral identification codes (<save area>\$<spectral id>).

For domain SIXTEEN_CHARACTERS:

Enter the data type for this domain (char/date/int/real): c

Enter the length of the character domain: 16

Enter the default value (return says NULL):

Enter any desired comment: Domain for sixteen-alphanumeric-long fields.

For domain SIXTY_CHARACTERS:

Enter the data type for this domain (char/date/int/real): c

Enter the length of the character domain: 60

Enter the default value (return says NULL):

Enter any desired comment: Domain for sixty-alphanumeric-long fields. For domain TEN_CHARACTERS:

Enter the data type for this domain (char/date/int/real): c

Enter the length of the character domain: 10

Enter the default value (return says NULL):

Enter any desired comment: Domain for ten-alphanumeric-long fields. For domain TWENTY_CHARACTERS:

Enter the data type for this domain (char/date/int/real): c

Enter the length of the character domain: 20

Enter the default value (return says NULL):

Enter any desired comment: Domain for twenty-alphanumeric-long fields.

For domain UNITS:

Enter the data type for this domain (char/date/int/real): c

Enter the length of the character domain: 5 
Enter the default value (return says NULL):

Enter any desired comment: Domain for five-alphanumeric-long units. For table SAMPLE_INFORMATION:

Enter any desired comment: Table for sample information.

Enter the number of rows per page to be clustered together: 1

Enter the maximum number of rows for this table: $\mathbf{4 0 0 0}$

For table ALPHA_URANIUM:

Enter any desired comment: Table for requested alpha analyseg for Uranium radionuclides.

For foreign key SAMPLE_TRACKING_ID:

Enter the reference table: SAMPLE_INFORMATION

Enter the reference column: SAMPLE_TRACKING_ID

Enter the number of rows per page to be clustered together: 1

Enter the maximum number of rows for this table: 1000

For table ALPHA_THORIUM:

Enter any desired comment: Table for requested alpha analyses for Thorium radionuclides.

For foreign key SAMPLE_TRACKING_ID:

Enter the reference table: SAMPLE_INFORMATION

Enter the reference column: SAMPLE_TRACRING_ID

Enter the number of rows per page to be clustered together: 1

Enter the maximum number of rows for this table: 1000

For table ALPHA_PLUTONIUM:

Enter any desired comment: Table for requested alpha analyses for plutonium radionuclides.

For foreign key SAMPLE_TRACKING_ID:

Enter the reference table: SAMPLB_INFORMATION

Enter the reference column: SAMPLE_TRACKING_ID

Enter the number of rows per page to be clustered together: 1

Enter the maximum number of rows for this table: 1000

For table ALPHA_AM241_SANS_PU238:

Enter any desired comment: Table for requested alpha analyses for 
Americium-241 separate from Plutonium-238.

For foreign key SAMPLE_TRACKING_ID:

Enter the reference table: SAMPLB_INFORMATION

Enter the reference column: SAMPLB_TRACKING_ID

Enter the number of rows per page to be clustered together: 1

Enter the maximum number of rows for this table: 1000

For table ALPHA_AM241_WITH_PU238:

Enter any desired comment: Table for requested alpha analyses for Americium-241 combined with Plutonium-238.

For foreign key SAMPLE_TRACKING_ID:

Enter the reference table: SAMPLE_INFORMATION

Enter the reference column: SAMPLE_TRACKING_ID

Enter the number of rows per page to be clustered together: 1

Enter the maximum number of rows for this table: 1000

For table ALPHA_TOTAL_SPECTROMETRIC:

Enter any desired comment: Table for requested total spectrometric alpha analyses.

For foreign key SAMPLE_TRACKING_ID:

Enter the reference table: SAMPLE_INFORMATION

Enter the reference column: SAMPLB_TRACKING_ID

Enter the number of rows per page to be clustered together: 1

Enter the maximum number of rows for this table: 1000

For table ALPHA_OTHER:

Enter any desired comment: Table for requested alpha analyses for other, unlisted radionuclides.

For foreign key SAMPLE_TRACKING_ID:

Enter the reference table: SAMPLB_INFORMATION

Enter the reference column: SAMPLE_TRACKING_ID

Enter the number of rows per page to be clustered together: 1

Enter the maximum number of rows for this table: 1000

For table BETA_STRONTIUM_90:

Enter any desired comment: Table for requested beta analyses for 


\section{Strontium-90.}

For foreign key SAMPLE_TRACKING_ID:

Enter the reference table: SAMPLE_INFORMATION

Enter the reference column: SAMPLE_TRACKING_ID

Enter the number of rows per page to be clustered together: 1

Enter the maximum number of rows for this table: 1000

For table BETA_STRONTIUM_89_AND_90:

Enter any desired comment: Table for requested beta analyses for strontium-89 and -90 .

For foreign key SAMPLE_TRACKING_ID:

Enter the reference table: SAMPLE_INFORMATION

Enter the reference column: SAMPLE_TRACKING_ID

Enter the number of rows per page to be clustered together: 1

Enter the maximum number of rows for this table: 1000

For table BETA_TOTAL_STRONTIUM:

Enter any desired comment: Table for requested beta analyses for total strontium.

For foreign key SAMPLE_TRACKING_ID:

Enter the reference table: SAMPLE_INFORMATION

Enter the reference column: SAMPLB_TRACKING_ID

Enter the number of rows per page to be clustered together: 1

Enter the maximum number of rows for this table: 1000

For table BETA_TRITIUM: Tritium.

Enter any desired comment: Table for requested beta analyses for

For foreign key SAMPLE_TRACKING_ID:

Enter the reference table: SAMPLB_INFORMATION

Enter the reference column: SAMPLB_TRACKING_ID

Enter the number of rows per page to be clustered together: 1

Enter the maximum number of rows for this table: 1000

For table BETA_OTHER:

Enter any desired comment: Table for requested beta analyses for 
other, unlisted radionuclides.

For foreign key SAMPLE_TRACKING_ID:

Enter the reference table: SAMPLE_INFORMATION

Enter the reference column: SAMPLE_TRACKING_ID

Enter the number of rows per page to be clustered together: 1

Enter the maximum number of rows for this table: 1000

For table GAMMA_SCREEN:

Enter any desired comment: Table for requested screening and shipping gamma analyses.

For foreign key SAMPLE_TRACKING_ID:

Enter the reference table: SAMPIE_INRORMATION

Enter the reference column: SAMPLB_TRACKING_ID

Enter the number of rows per page to be clustered together: 1

Enter the maximum number of rows for this table: $\mathbf{4 0 0 0}$ For table GAMMA_FULL_ISOTOPIC:

Enter any desired comment: Table for requested full isotopic gamma analyses.

For foreign key SAMPLE_TRACKING_ID:

Enter the reference table: SAMPLE_INFORMATION

Enter the reference column: SAMPLE_TRACKING_ID

Enter the number of rows per page to be clustered together: 1

Enter the maximum number of rows for this table: $\mathbf{4 0 0 0}$

For table GAMMA_OTHER:

Enter any desired comment: Table for requested gamma analyses for other, unlisted radionuclides.

For foreign key SAMPLE_TRACKING_ID:

Enter the reference table: SAMPLB_INFORMATION

Enter the reference column: SAMPLE_TRACKING_ID

Enter the number of rows per page to be clustered together: 1

Enter the maximum number of rows for this. table: $\mathbf{4 0 0 0}$

For table GROSS_ALPHA_BETA_AIR_FILTERS:

Enter any desired comment: Table for requested gross alpha-beta 
analyses of air filters.

For foreign key SAMPLE_TRACKING_ID:

Enter the reference table: SAMPLE_INFORMATION

Enter the reference column: SAMPLE_TRACKING_ID

Enter the number of rows per page to be clustered together: 1

Enter the maximum number of rows for this table: 1000

For table GROSS_ALPHA_BETA_OTHER:

Enter any desired comment: Table for requested gross alpha-beta analyses of other, unlisted samples.

For foreign key SAMPLE_TRACKING_ID:

Enter the reference table: SAMPLE_INFORMATION

Enter the reference column: SAMPLE_TRACKING_ID

Enter the number of rows per page to be clustered together: 1

Enter the maximum number of rows for this table: 1000

For table SPECIAI_INSTRUCTIONS:

Enter any desired comment: Table for special instructions.

For foreign key SAMPLE_TRACKING_ID:

Enter the reference table: SAMPLE_INEORMATION

Enter the reference column: SAMPLE_TRACKING_ID

Enter the number of rows per page to be clustered together: 15

Enter the maximum number of rows for this table: 20000

For table TRACKING:

Enter any desired comment: Table for tracking samples.

For foreign key SAMPLE_TRACKING_ID:

Enter the reference table: SAMPLB_INFORMATION

Enter the reference column: SAMPLE_TRACKING_ID

Enter the number of rows per page to be clustered together: 5

Enter the maximum number of rows for this table: 20000

Executicr. has succesミzully completed. The create_schema.sql and cursor and . ssert filez iill be closed and retained. The entity and screen files will also be closed and retained.

Please wait while certain sections of the cursor and insert files are 
rearranged; this may take a few minutes.

Cursor file rearranged.

Insert file rearranged.

$\$$ 


\section{DATABASE BACKUP - AN EXCERPT FROM THE RML OPERATOR'S BACKUP ROUTINE}

Note the closed sets of ellipses in the two "set default" lines below; these would be replaced with the appropriate directory (\$USER:[DATABASES.FILES], for example).

\$! Prior to performing the DCL BACKUP, backup the contents of the

$\$$ ! sample tracking database.

$\$ !$

$\$$ write sys\$̧output ".

$\$$ write sys\$output "Verifying the integrity of the database . . ."

$\$$ write sys\$output

$\$$ set default ...

$\$ \mathrm{rmu} / v e r i f y$ sample_tracking !Verify the integrity of the database.

$\$$ write sys\$output "

$\$$ write sys\$output "Preparing a version of the database which can be backed up . . ."

$\$$ write sys \$output " "

\$ rmu/backup sample_tracking sample_tracking !Backup the database.

$\$$ write sys\$output " "

$\$$ write sys\$output "Database successfully verified and prepared."

$\$$ write sys\$output "

$\$ !$

$\$$ ! Purge most prior versions of the database backup file, and mark

$\$$ ! those that remain for future "no backup."

$\$ !$

$\$$ write sys\$output " "

$\$$ write sys \$output "Purging old backup versions of the database . . ."

$\$$ write sys\$output " "

$\$$ set default ...

$\$$ purge/keep $=2$ sample_tracking.rbf

$\$$ set file sample_tracking.rbf; * /nobackup 


\section{E. ARRAY AND SCALAR DECLARATIONS RELATED TO THE SCREEN MANAGEMENT ROUTINES}

C

C C

C

C

C

C

C

C

C

$\mathrm{C}$

C

C

C

$\mathrm{C}$
$\mathrm{C}$

$\mathrm{C}$
$\mathrm{C}$

$\mathrm{C}$

C

C

C

Declaration statements.

Component of SAMPLE_TRACKING Version 1 completed June 6, 1991 by D. A. Femec.

Note that variable names are limited to six-characters in length; this is a limitation imposed by SQL for variables used in SQL calls.

Field rendition descriptions.

integer*4 ferror/smg\$m_blink/, !For errors in field entries.

- ffixed/smg\$m_reverse/.' !For fixed fields.

- finput/smg\$m_underline/, ! For input fields.

- foptnl/smg\$m_normal/, !For optional fields.

- freqd/smg\$m_bold/ ! For required fields.

common /fldscr/ ferror, ffixed, finput, foptnl, freqd

Main screen's field descriptors and field sizes.

character $* 80$ fieldM (36), fidatM(36), findat,blanks

character $* 30$ atrbtM $(2,38)$

character $* 14$ varblm(38)

character $* 1$ spaces $(80) / 80 * \prime /$, fitypM(36)

integer*4 fdlenM(36), farowm(36), fdcolm(36), ficolm(36), $\operatorname{mxnocm}(36)$,

- fdrndM $(3,36)$, firndM(3,36), ! Field renditions (add/update/search).

- numfdM $/ 36 /$ !The number of fields.

integer*2 ifnulM(36), !Null indicator (-1 if null, 0 otherwise).

- ifrqdM(36) $/ 36 * 0 \%$ ! Points to if-required dependent field.

- iditom(36) $/ 36 * 0 /$ ! Points to allowed field for dittoing.

logical ifmodM(36) ! Has a field been modified in an Update? common /dbdatM/ fieldM, fidatM, fdlenM, fdrowM, fdcolM, ficolM, mxnocM,

- fdrndM, firndM, numfdM, fitypM, ifnulM, atrbtM, varblM, ifrqdM, ifmodM,

- iditom

equivalence (blanks (1:1), spaces (1))

Length-specified character fields for equivalences ( $f$ for field).

character fMSTI*12, fMDSE*6, fMDAWC *6, fMCSI* $12, \mathrm{fMRNBD} * 6, \mathrm{fMCPN} * 40$,

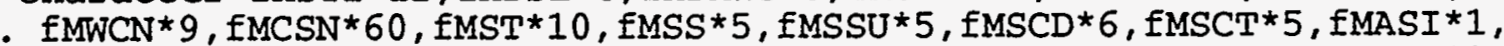

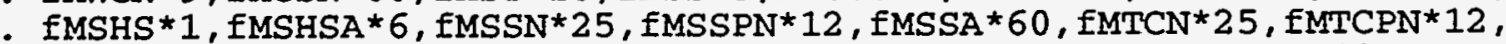

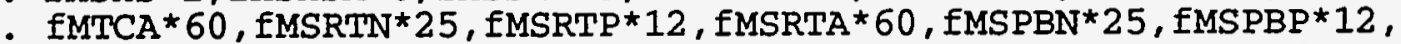

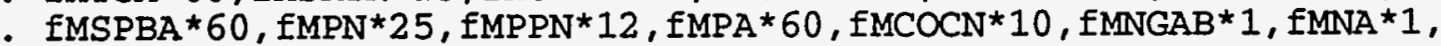

- $\mathrm{EMNB} * 1$, $\mathrm{EMNG} * 1$

Real and integer field storage (r for real, $i$ for integer).

real* 4 rMSS, rMSHSA

integer * 4 iMDSE, IMDAWC, IMRNBD, IMSCD

- ,nxinst, nxcust !Declared in the main routine.

Nonarrayed field lengths ( 1 for lengths).

integer *4 1MSTI, IMDSE, IMDAWC, IMCSI , 1MRNBD, IMCPN, IMWCN, IMCSN, 1MST, - IMSS, IMSSU, IMSCD , IMSCT , IMASI, IMSHS, IMSHSA, IMSSN, IMSS PN, IMSSA, 
- 1MTCN, IMTCPN, 1MTCA, IMSRTN, 1MSRTP, 1MSRTA, IMSPBN, 1MSPBP, 1MSPBA,

IMPN , IMPPN, IMPA, IMCOCN , IMNGAB , IMNA, IMNB, IMNG

Nonarrayed null indicators for data transfers (n for nul1).

integer*2 nMSTI, nMDSE, nMDAWC, nMCSI, nMRNBD, nMCPN, nMWCN, nMCSN, nMST,

- nMSS, nMSSU, nMSCD , nMSCT , nMASI , nMSHS, nMSHSA, nMSSN, nMSSPN, nMSSA,

- $\mathrm{nMTCN}, \mathrm{nMTCPN}, \mathrm{nMTCA}, \mathrm{nMSRTN}$, nMSRTP , nMSRTA, nMSPBN , nMSPBP, nMSPBA,

$\mathrm{C}$

- nMPN, nMPPN , nMPA, nMCOCN , nMNGAB, nMNA, NMNB, nMNG

C

C

Nonarrayed if-modified indicators (m for modified).

integer *2 mMSTI, MMDSE, MMDAWC , MMCSI , MMRNBD, MMCPN , MMWCN , MMCSN , mMST,

- mMSS, MMSSU, MMSCD , MMSCT , MMASI, mMSHS , MMSHSA, MMSSN, MMSSPN , MMSSA,

- $\mathrm{MMTCN}$, MMTCPN , MMTCA , MMSRTN, MMSRTP , MMSRTA, IMMSPBN, MMSPBP, MMSPBA,

- $\mathrm{MMPN}$, MMPPN , MMPA, MMCOCN , MMNGAB , MMNA , MMNB , MMNG

Equivalence statements for data transfers.

equivalence (fidatM(1) (1:12), fMSTI), (ifnulM(1), nMSTI),

- (fidatM(2) (1:6), fMDSE), (ifnulM(2), nMDSE),

- (fidatm(3) (1:6), IMDAWC), (ifnulM(3), nMDAWC),

- (fidatm(4) (1:12), fMCSI), (ifnulM(4), nMCSI),

- (fidatM(5) (1:6), fMRNBD), (ifnulM(5), nMRNBD),

- (fidatM (6) $(1: 40)$, fMCPN), (ifnulM(6), nMCPN),

- (fidatM (7) (1:9), fMWCN), (ifnulM(7), nMWCN),

- (fidatm(8) (1:60), fMCSN), (ifnulm(8), nMCSN),

- (fidatM(9) (1:10), fMST), (ifnulM(9), nMST),

- (fidatm(10) (1:5), fMSS), (ifnulM(10), nMSS),

- (fidatm(11) (1:5), fMSSU), (ifnulM(11), nMSSU),

- (fidatM(12) (1:6), fMSCD), (ifnulM(12), nMSCD),

- (fidatm(13) (1:5), fMSCT), (ifnulm(13), nMSCT),

- (fidatM(14) (1:1), IMASI), (ifnulm(14), nMASI),

- (fidatm(15) (1:1), fMSHS), (ifnulm(15), nMSHS),

- (fidatm(16) (1:6), fMSHSA), (ifnulm(16), nMSHSA),

- (fidatm(17) (1:25), fMSSN), (ifnulM(17), nMSSN),

- (fidatM (18) (1:12), fMSSPN), (ifnulM(18), nMSSPN),

- (fidatm(19) (1:60), IMSSA), (ifnulm(19), nMSSA),

(fidatm(20) (1:25), fMTCN), (ifnulM(20), nMTCN),

- (fidatm(21) (1:12), fMTCPN), (ifnulm(21), nMTCPN),

- (fidatM(22) (1:60), EMTCA), (ifnulM(22), nMTCA),

- (fidatm(23) (1:25), fMSRTN),(ifnulM(23), nMSRTN),

- (fidatm(24) (1:12), fMSRTP), (ifnulM(24), nMSRTP),

- (fidatM(25) (1:60), IMSRTA), (ifnulM(25), nMSRTA),

- (fidatM(26) (1:25), fMSPBN), (ifnulM(26), nMSPBN),

- (fidatm(27) (1:12), fMSPBP), (ifnulM(27),nMSPBP),

- (fidatm(28) (1:60), fMSPBA), (ifnulm(28), nMSPBA),

- (fidatM(29) (1:25), , IMPN), (ifnulM(29), nMPN),

- (fidatm(30) (1:12), fMPPN), (ifnulM(30),nMPPN),

- (fidatm(31) (1:60), fMPA), (ifnulm(31), nMPA),

- (fidatM(32) (1:10), IMCOCN), (ifnulM(32), nMCOCN),

- (fidatm(33) (1:1), fMNGAB), (ifnulM(33), nMNGAB),

- (fidatM(34) (1:1), fMNA), (ifnulM(34), nMNA),

- (fidatM(35) (1:1), fMNB), (ifnulM(35), nMNB),

- (fidatM(36) (1:1), fMNG), (ifnulM(36),nMNG),

- (mxnocM (1), IMSTI), (ifmodM(1), mMSTI),

- (mXnocm (2), 1MDSE), (ifmodM(2), mMDSE),

- (mXnocm (3), 1MDAWC), (ifmodM (3), mMDAWC),

- (mXnocM (4), IMCSI)., (ifmodM(4), mMCSI),

- (mXnocM (5), IMRNBD), (ifmodM (5), MMRNBD), 
- (mxnocM( 6$), 1 M C P N),(i \operatorname{imodM}(6), \operatorname{mMCPN})$,

- (mXnocM(7), IMWCN), (i $\operatorname{modM}(7), \operatorname{mMWCN})$,

- (mxnocM(8), IMCSN), (ímodM(8), mMCSN),

- (mxnocM (9), lMST), (ifmodM (9), mMST),

- (mXnocM(10), IMSS)", (i fmódM (10)',mMSS),

- (mxnocm(11), IMSSU), (ifmodM (11), mMSSU),

- (mxnocm (12), 1MSCD), (ifmodM (12), mMSCD),

- (mxnocm (13), IMSCT), (ifmodM (13), mMSCT),

- (mxnocm(14), IMASI), (i.fmodM (14), mMASI),

- (mxnocm(15), 1MSHS), (ifmodM(15), mMSHS),

- (mxnocM (16), IMSHSA), (ifmodM(16), mMSHSA),

- (mxnocm (17), IMSSN), (ifmodM (17), mMSSN),

- (mXnocM(18), IMSSPN), (i $\operatorname{imodM}(18), \operatorname{mMSSPN})$,

- (mXnocm(19), IMSSA), (ifmodM (19), mMSSA),

- (mixnocM(20), IMTCN), (ifmodM(20), mMTCN),

- (mXnocM (21), IMTCPN), (i $\operatorname{modM}(21), \operatorname{mMTCPN})$,

- (mXnocm (22), IMTCA), (ifmodM (22), mMTCA),

- (mXnocM (23), IMSRTN), (i fmodM(23), mMSRTN),

- (mXnocM (24), 1MSRTP), (i fmodM (24), mMSRTP),

- (mXnOcM (25), IMSRTA), (ifmodM(25), MMSRTA),

- (mXnocM (26), IMSPBN), (i $\operatorname{modM}(26)$, mMSPBN),

- (mxnocm (27), IMSPBP), (i $\operatorname{modm}(27)$, mMSPBP),

- (mXnocm(28), IMSPBA), (i fmodM(28), mMSPBA),

- (mXnocm(29), IMPN), (i $\operatorname{modm}(29)$, mMPN) ,

- (mXnocm(30), IMPPN), (ifmodM(30), mMPPN),

- (mxnocm(31), IMPA), (i $\operatorname{modm}(31), \operatorname{mMPA})$,

- (mXnOcM(32), IMCOCN), (i $\operatorname{modM}(32), \operatorname{mMCOCN})$,

- (mXnocM(33), IMNGAB), (i $\operatorname{imodM}(33), \operatorname{mMNGAB})$,

- (mXnocM (34), IMNA), (i $\operatorname{imodM}(34)$, mMNA),

- (mXnocM (35), IMNB), (i $\operatorname{modM}(35)$, mMNB),

C

- (mxnocm(36), IMNG), (ifmodM(36), MMNG)

C Database table and attribute names for screen fields.

data atrbtm/

.' 'SAMPLE_INFORMATION','SAMPLE_TRACKING_ID',

- 'SAMPLE_INFORMATION' , 'DATE_SĀMPLE_ENTERED',

- 'SAMPLE_INFORMATION', 'DATE_ALL_WORK_COMPLETED',

- 'SAMPLE_INFORMATION', 'CUSTOMER_SAMPLE_ID',

- 'SAMPLE_INFORMATION',' RESULTS_NEEDED_BY_DATE',

- 'SAMPLE_INFORMATION', 'CUSTOMER_PROJECT_NAME',

- 'SAMPLE_INFORMATION' , 'WORK_CHARGE_NUMBER',

- 'SAMPLE_INFORMATION' ' CUSTOMER_SAMPLE_NAME',

- 'SAMPLE_INFORMATION' , SAMPLE_TYPE',

'SAMPLE_INFORMATION',' SAMPLE_SIZE',

- 'SAMPLE_INFORMATION' ,'SAMPLE_SIZE_UNITS',

- 'SAMPLE INFORMATION', 'SAMPLE COLIECTION DATE',

- 'SAMPLE_INFORMATION', 'SAMPLE_COLLECTION_TIME',

- 'SAMPLE_INFORMATION' , 'ANY_SPECIAL_INSTRUCTIONS',

- 'SAMPLE_INFORMATION',' SAMPLE_HP_SURVEYED',

- 'SAMPLE_INFORMATION' ' SAMPLE_HP_SURVEY_ACTIVITY',

- 'SAMPLE_INFORMATION', 'SAMPLE_SUBMITTER_NAME' ,

- 'SAMPLE_INFORMATION' , 'SAMPLE_SUBMITTER_PHONE_NUMBER' ,

- 'SAMPLE_INFORMATION', 'SAMPLE_SUBMITTER_ADDRESS',

'SAMPLE_INFORMATION', 'TECHNICAL_CONTACT_NAME',

' 'SAMPLE_INFORMATION' , 'TECHNICAL_CONTACT_PHONE_NUMBER' ,

- 'SAMPLE_INFORMATION' ' TECHNICAL_CONTACT_ADDRESS',

- 'SAMPLE_INFORMATION', 'SEND_RESULTS_TO_NAME',

- 'SAMPLE_INFORMATION' ' 'SEND_RESULTS_TO_PHONE_NUMBER',

- 'SAMPLE_INFORMATION','SEND_RESULTS_TO_ADDRESS', 
- 'SAMPLE_INFORMATION','SAMPLE_PICKUP_BY_NAME',

- 'SAMPLE_INFORMATION', 'SAMPLE_PICKUP_BY_PHONE_NUMBER',

- 'SAMPLE_INFORMATION', 'SAMPLE_PICKUP_BY_ADDRESS',

'TRACKING' , 'POSSESSOR_NAME',

'TRACKING' , 'POSSESSOR_PHONE_NUMBER',

'TRACKING' ,' POSSESSOR_ADDRESS',

'SAMPLE_INFORMATION' 'CHAIN_OF_CUSTODY_NUMBER',

'SAMPLE_INFORMATION', 'NEED_GROSS_ALPHA_BETA',

'SAMPLE_INFORMATION', 'NEED_ALPHA',

'SAMPLE_INFORMATION', 'NEED_BETA',

'SAMPLE_INFORMATION'', 'NEED_GAMMA',

'SAMPLE_INFORMATION','NEXT_SPECIAI_INSTRUCTION_LINE' ,

'SAMPLE_INFORMATION',' 'NEXT_POSSESSOR_SEQUENCE_NUMBER' /

C

Program variable names (value and null indicator) for screen fields.

data varblM/': fMSTI :nMSTI',': iMDSE:nMDSE',' : IMDAWC: NMDAWC',

' ' : fMCSI: nMCSI',' : iMRNBD: nMRNBD', ': fMCPN:nMCPN',' : fMWCN:nMWCN',

- ' fMCSN:nMCSN', ' :fMST: nMST',' : rMSS:nMSS', ' : fMSSU:nMSSU',

- ' : iMSCD:nMSCD',' : fMSCT:nMSCT', ' : EMASI:nMASI', ': EMSHS:nMSHS',

- ': 1 MSHSA:nMSHSA' , ' : fMSSN: nMSSN' , ' IMSSPN:nMSSPN', ': IMSSA:nMSSA',

- ' : IMTCN: nMTCN', ' : IMTCPN:nMTCPN', ' : IMTCA:nMTCA', ' : fMSRTN : nMSRTN',

. ' : fMSRTP:nMSRTP', ' : fMSRTA:nMSRTA', ' : fMSPBN:nMSPBN',

- ' : fMSPBP : nMSPBP', ' : IMSPBA:nMSPBA', ' : fMPN:nMPN',

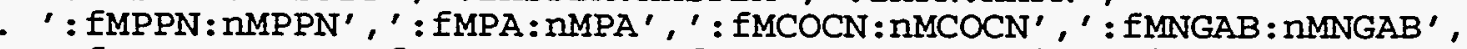

' ' fMNA : nMNA',' : fMNB:nMNB', ': fMNG : nMNG',' :nxinst : ifnnxi',

- ' nxcust: ifnnxc'/

Alpha screen's field descriptors and field sizes.

character*80 fieldA (37), fidatA(37)

character*30 atrbtA $(2,37)$

character $* 14$ varbla (37)

character $* 1$ fitypa (37)

integer*4 fdlenA (37), fdrowa (37), fdcola (37), ficola (37), mxnocA (37),

- fdrndA (3,37), firndA $(3,37)$, ! Field renditions (add/update/search).

- numfdA/37/ ! The number of fields.

integer*2 ifnulA(37), !Null indicator (-1 if null, 0 otherwise).

- ifrqdA (37) $/ 37 * 0 /$, Points to if-required dependent field.

- iditoA(37) $/ 37 * 0 /$ ! Points to allowed field for dittoing.

logical ifmodA(37) ! Has a field been modified in an Update?

common /dbdatA/ fieldA, fidatA, fdlenA, fdrowA, fdcolA, ficolA, mxnocA,

- fdrndA, firndA, numfdA, fitypA, ifnulA, atrbtA, varblA, ifrqdA, ifmodA,

- iditoA

Length-specified character fields for equivalences (f for field).

character fASTI* 12, fACSN $* 60$, fANAU $* 1$, fARNB $1 * 6$, fADAW $1 * 6$, fARRC $1 * 20$,

- fAASI $1 * 1$, fANATh $* 1$, fARNB $2 * 6$, fADAW $2 * 6$, fARRC $2 * 20$, fAASI $2 * 1$, fANAPu $* 1$,

- fARNB $3 * 6$, fADAW $3 * 6$, fARRC $3 * 20$, fAASI $3 * 1$, fANASP $* 1$, fARNB $4 * 6$, fADAW $4 * 6$,

. fARRC4 $* 20$, fAASI $4 * 1$, fANAWP $* 1$, fARNB $5 * 6$, fADAW5 56, fARRC $5 * 20$, fAASI $5 * 1$,

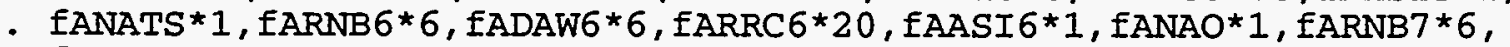

- IADAW7*6, fARRC $7 * 20$, fAASI $7 * 1$

Integer field storage ( $i$ for integer).

integer *4 IARNB1, iADAW1, IARNB2, iADAW2, iARNB3 , IADAW3, iARNB4, iADAW4,

- iARNB5, iADAW5, IARNB6, iADAW6, IARNB7, iADAW7

Nonarrayed maximum field lengths (1 for length). 
integer *4 1ASTI, IACSN, IANAU, 1ARNB1, IADAW1, 1ARRC1, 1AASI1, IANATh, - IARNB2 , IADAW2, IARRC2, IAASI2, IANAPU, IARNB3, IADAW3, IARRC3, IAASI3, - IANASP, IARNB4, IADAW4, IARRC4, IAASI4, IANAWP, IARNB5, IADAW5, IARRC5, - IAASI5, IANATS, IARNB6, IADAW6; IARRC6, IAASI6, IANAO, IARNB7, IADAW7,

- IARRC7, IAASI7

$\mathrm{C}$

C Nonarrayed null indicators for data transfers (n for null).

integer *2 nASTI, nACSN, nANAU, nARNB1, nADAW1, nARRC1, nAASI1, nANATh, - nARNB2 , nADAW2 , nARRC2, nAASI2, nANAPU, nARNB3, nADAW3, nARRC3, nAASI3, - nANASP, nARNB4, nADAW4, nARRC4, nAASI4, nANAWP, nARNB5, nADAW5, nARRC5, - nAASI5, nANATS, nARNB6, nADAW6, nARRC6, nAASI6, nANAO, nARNB7, nADAW7, - nARRC7, nAASI7

$\mathrm{C}$
$\mathrm{C}$
$\mathrm{C}$

Nonarrayed if-modified indicators (m for modified).

integer *2 mASTI, MACSN , MANAU, MARNB1, MADAW1, MARRC1, MAASI1, MANATh, - MARNB2 , MADAW2 , MARRC2 , MAASI2, MANAPU, MARNB3, MADAW3 , MARRC3 , MAASI3, - MANASP , MARNB4, MADAW4 , MARRC4, MAASI4 , MANAWP , MARNB5, mADAW5, mARRC5, - MAASI5, mANATS, MARNB6, MADAW6, MARRC6, MAASI6, MANAO, mARNB7, MADAW7, - marRc7,maAsi7

C Equivalence statements for data transfers.

equivalence (fidata(1) ( $1: 12$ ), fASTI), (ifnula (1), nASTI), - (fidata (2) $(1: 60)$, fACSN), (ifnula (2), nACSN),

- (fidata (3) (1:1), EANAU), (ifnula (3), nANAU),

- (fidata (4) $(1: 6)$, farNB1), (i fnula (4), MARNB1),

- (fidata (5) (1:6), EADAW1), (i fnula (5), nADAW1),

- (fidata (6) (1:20), faRRC1), (ifnula(6), nARRC1),

- (fidata (7) (1:1), EAASI1), (ifnula (7), nAASI1),

- (fidata (8) (1:1), fANATh), (ifnula (8), nANATh),

- (fidata (9) $(1: 6)$, fARNB2), (i fnula (9), nARNB2),

- (fidata (10) (1:6), fADAW2), (ifnula (10), nADAW2),

- (fidatA(11) (1:20), fARRC2), (ifnulA (11), nARRC2),

- (fidatA (12) (1:1), fAASI2), (ifnula (12), nAASI2),

- (fidata (13) (1:1), fANAPu), (ifnula (13), nANAPu),

- (fidata (14) (1:6), fARNB3), (ifnula (14), nARNB3),

- (fidata (15) (1:6), faDAW3), (ifnula (15), nADAW3),

- (fidata (16) (1:20), fARRC3), (ifnula (16), nARRC3),

- (fidata (17) (1:1), fAASI3), (ifnula (17), nAASI3),

- (fidata (18) (1:1), fANASP), (ifnula (18), nANASP),

- (fidata (19) (1:6), fARNB4), (ifnula (19), nARNB4),

- (fidata (20) (1:6), fADAW4), (ifnula (20), nADAW4),

- (fidata (21) (1:20), fARRC4), (ifnula (21), nARRC4),

- (fidata (22) (1:1), fAASI4), (ifnula (22), nAASI4),

- (fidata (23) (1:1), faNAWP), (ifnula (23), nANAWP),

- (fidatA (24) (1:6), fARNB5), (ifnula (24), nARNB5),

- (fidatA (25) (1:6), fADAW5), (ifnula (25), nADAW5),

- (fidata (26) (1:20), fARRC5), (i fnula (26), nARRC5),

- (fidata (27) (1:1), fAASI5), (ifnula (27), nAASI5),

- (fidata (28) (1:1), fanATS), (ifnula (28), nANATS),

- (fidata (29) $(1: 6)$, faRNB6), (ifnula (29), nARNB6),

- (fidata (30) (1:6), faDAW6), (ifnula (30), naDAW6),

- (fidata (31) (1:20), fARRC6), (ifnula (31), nARRC6),

- (fidata (32) (1:1), faAsI6), (ifnula (32), naASI6),

- (fidata (33) (1:1), faNAO), (ifnula (33), nanaO),

- (fidata (34) (1:6), fARNB7), (ifnula (34), nARNB7),

- (fidata (35) (1:6), fADAW7), (ifnula (35), nADAW7), 
- (fidata (36) (1:20), fARRC7),(i亡: 1A(36),nARRC7),

- (fidatA (37) (1:1), fAASI7), (ifn . A(37), nAASI7),

- (mxnoca (1), IASTI), (ifmodA (1), I STI),

- (mXnoca (2), IACSN), (i $\operatorname{mmodA}(2)$, MACSN),

- (mXnoca (3), IANAU), (i imoda (3), manaU),

- (mXnOcA (4), IARNB1), (ifmoda (4), maRNB1),

- (mxnoca (5), IADAW1), (i fmoda (5), maDAW1),

- (mXnOcA (6), 1ARRC1), (i imoda (6), maRRC1),

- (mxnoca (7), IAASI1), (ifmoda (7), maASI1),

- (mXnOcA (8), IANATh), (ifmodA (8), maNATh),

- (mxnoca (9), IARNB2), (ifmoda (9), maRnB2),

- (mXnoca (10), IADAW2), (ifmoda (10), MADAW2),

- (mXnOcA (11), IARRC2), (ifmoda (11), MARRC2),

- (mxnoca (12), IAASI2), (ifmoda (12), maASI2),

- (mXnoca (13), lanapu), (i imoda (13), manaPu),

- (mXnOCA (14), IARNB3)', (i imoda (14), MARNB3),

- (mXnOcA (15), IADAW3), (ifmodA (15), maDAW3),

- (mMOCA (16), IARRC3), (ifmoda (16), maRRC3),

- (r IOCA (17), IAASI3), (ifmoda (17), mAASI3),

- (n nocA (18), IANASP), (ifmodA (18), MANASP),

- (mXnOCA (19), IARNB4), (i imoda (19), maRNB4),

- (mXnOcA (20), IADAW4), (i imoda (20), MADAW4),

- (mXnOca (21), IARRC4), (i fmoda (21), marRC4),

- (mXnoca (22), IAASI4), (i imoda (22), maASI4),

- (mXnOcA (23), IANAWP), ( i fmodA (23), MANAWP),

- (mxrioca (24), IARNB5), (i fmoda (24), maRNB5),

- (mXnOcA (25), IADAW5), (ifmoda (25), maDAW5),

- (mXnOcA (26), IARRC5), (ifmoda (26), maRRC5),

- (mXnOcA (27), IAASI5), (ifmodA (27), MAASI5),

- (mXnOcA (28), IANATS), (i fmoda (28), manats),

- (mXnOcA (29), IARNB6), (i fmoda (29), MARNB6),

- (mXnoca (30), ilaDAW6), (i fmoda (30), maDAW6),

- (mXnOCA (31), IARRC6), (ifmoda (31), MARRC6),

- (mxnoca (32), IAASI6), (ifmoda (32), maAsI6),

- (mXnOCA (33), IANAO), (ifmodA (33), manaO),

- (mXnOcA (34), IARNB7), (ifmoda (34), maRNB7),

- (mXnOcA (35), IADAW7), (i fmoda (35), maDAW7),

- (mXnOCA (36), IARRC7), (i imoda (36), mARRC7),

- (mXnocA (37), IAASI7), (i fmodA (37), mAASI7)

C Database table and attribute names for screen fields.

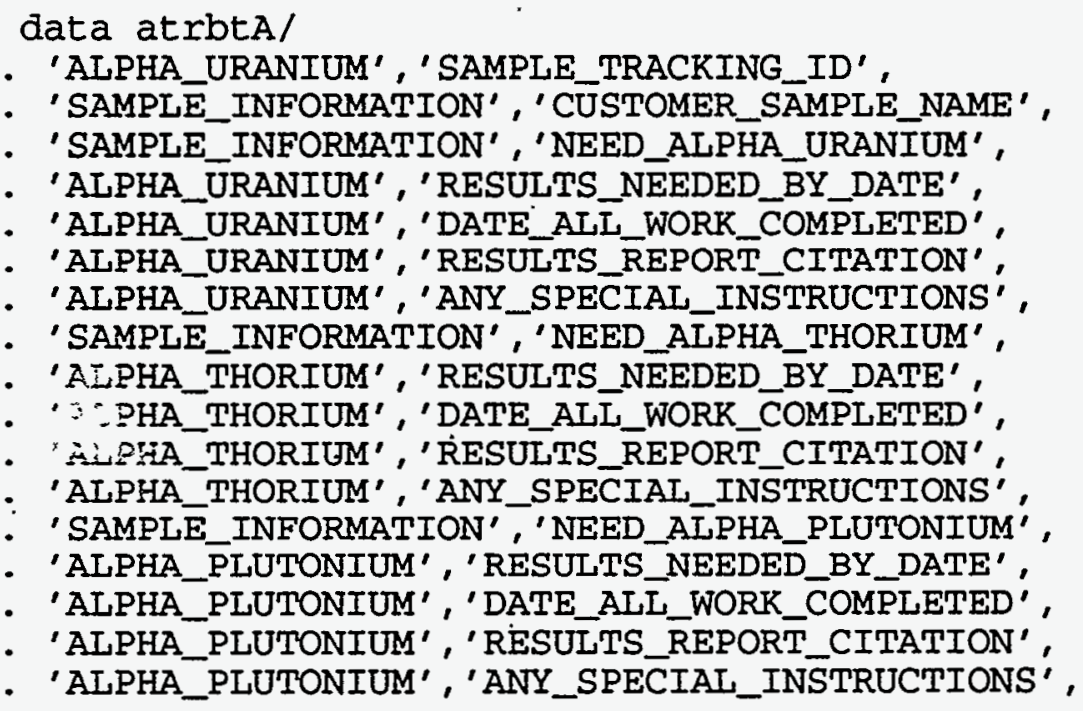


. 'SAMPLE_INFORMATION', 'NEED_ALPHA_AM241_SANS_PU238',

'ALPHA_AM241_SANS_PU238', 'RESULTS_NEEDED_BY_DATE',

'ALPHA_AM241_SANS_PU238', 'DATE_ALI_WORK_COMPLETED' ,

'ALPHA_AM241_SANS_PU238','RESULTS_REPORT_CITATION',

'ALPHA_AM2 41_SANS_PU238', 'ANY_SPECIAL_INSTRUCTIONS' ,

'SAMPLE_INFORMATION' , 'NEED_ALPHA_AM241_WITH_PU238',

'ALPHA_AM241_WITH_PU238', 'RESULTS_NEEDED_BY_DATE',

'ALPHA_AM241_WITH_PU238','DATE_ALI_WORK_COMPLETED',

'ALPHA_AM2 41_WITH_PU238','RESULTS_REPORT_CITATION',

'ALPHA_AM241_WITH_PU238', 'ANY_SPECIAL_INSTRUCTIONS',

'SAMPLE_INFORMATION' , 'NEED_ALPHA_TOTAL_SPECTROMETRIC',

'ALPHA_TOTAL_SPECTROMETRIC', 'RESULTS_NEEDED_BY_DATE',

'ALPHA_TOTAL_SPECTROMETRIC', 'DATE_ALI_WORK_COMPLETED',

'ALPHA_TOTAL_SPECTROMETRIC', 'RESULTS_REPORT_CITATION',

'ALPHA_TOTAL_SPECTROMETRIC', 'ANY_SPECIAI_INSTRUCTIONS',

' SAMPLE_INFORMATION' , 'NEED_ALPHA_OTHER',

'ALPHA_OTHER', 'RESULTS_NEEDED_BY_DATE',

'ALPHA_OTHER', 'DATE_ALI_WORK_COMPLETED',

'ALPHA_OTHER', 'RESULTS_REPORT_CITATION',

'ALPHA_OTHER', 'ANY_SPECIAL_INSTRUCTIONS' /

C

C

C

$\mathrm{C}$

C

$\mathrm{C}$

C

C

C

Program variable names (value and null indicator) for screen fields.

data varblA/' : fASTI:nASTI', ': fACSN:nACSN' ,' : fANAU: nANAU',

' : IARNB1 : nARNB1', ' : IADAW1: nADAW1', ' : fARRC1 : IARRCI',

' ' fAASI1 : IAASI1', ' : faNATh:nANATh' ,' : iARNB2 :nARNB2',

' : IADAW2 : IADAW2', ' : fARRC2 : IARRC2', ' : fAASI2 :nAASI2',

' : fANAPu : naNAPu', ' : iARNB3 : nARNB3 ', ' : iADAW3 : nADAW3',

' : fARRC3 : nARRC $31, '$ : fAASI 3 : nAASI3', ' : fANASP:nANASP',

' : IARNB4 : nARNB4', ' : IADAW4 : nADAW4', ' : fARRC4 : nARRC4',

' : fAASI4 : IAASI4', ' : faNAWP: nANAWP', ' : iARNB5 : nARNB5',

' : IADAW5 : nADAW5', ' : fARRC5:nARRC5',' : fAASI5:nAASI5',

$\therefore$ : fANATS : nANATS', ' : iARNB6: nARNB6', ' : iADAW6:nADAW6',

$'$ : fARRC6: IARRC6', ' : fAASI6: IAASI6',' : fANAO: nANAO',

- ' : iARNB7 : nARNB7', ' : iADAW7 : nADAW7',' : fARRC7:nARRC7',

' : fAASI7 : nAASI7' $/$

Beta screen's field descriptors and field sizes.

character $* 80$ fieldB $(46)$, fidat $B(46)$

character $* 30$ atrbtB $(2,46)$

character $* 14$ varblB. (46)

character*1 fitypB (46)

integer*4 fdlenB (46), fdrowB (46), fdcolB (46), ficolB (46), mxnocB (46),

- fdrndB $(3,46)$, firndB $(3,46)$, !Field renditions (add/update/search).

- numfdB/46/ !The number of fields.

integer*2 ifnulB(46), !Null indicator (-1 if null, 0 otherwise).

- ifrgaB (46)/46*0\%, ! Points to if-required dependent field.

- iditoB $(46) / 46 * 0 /$ ! Points to allowed field for dittoing.

logical ifmodB (46) ! Has a field been modified in an Update?

common /dbdat $B$ / fieldB, fidat $B$, fdlenB, fdrowB, fdcolB, ficolB, mxnocB,

- fdrndB, firndB, numfdB, fitypB, ifnulB, atrbt $B$, varblB, ifrqdB, ifmodB,

- iditoB

Length-specified character fields for equivalences ( $f$ for field).

character fBSTI*12, fBCSN*60, fBNBSr*1, fBDCL1*4, fBDCU1*1, fBRND 1 *6,

- fBRNT $1 * 5$, fBDAW $1 * 6$, fBRRC $1 * 20$, fBASI $1 * 1$, fBNBSC $* 1$, fBDCL $2 * 4$, fBDCU $2 * 1$,

- fBRND2 2 6, fBRNT2 *5, fBDAW2 *6, fBRRC2 *20, fBASI $2 * 1$, fBNBST*1, fBDCL $3 * 4$,

- fBDCU $3 * 1$, fBRND $3 * 6$, fBRNT3 3 , 5 , $\mathrm{fBDAW} 3 * 6$, fBRRC $3 * 20$, fBASI $3 * 1$, fBNBT $* 1$, 
- $\mathrm{fBDCL} 4$ * $4, \mathrm{fBDCU} 4$ *1, fBRND 4 *6, fBRNT4 *5, fBDAW4 *6, fBRRC4 *20,fBASI 4 * 1 ,

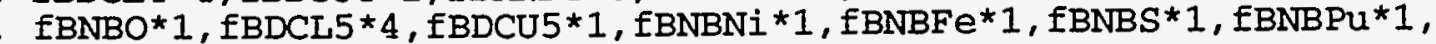

- EBRND5*6, fBRNT5*5, fBDAW5*6, fBRRC5*20, โBASI5*1

C

C

C

C

C

C

C

C

C

C

C

$\mathrm{C}$

C

C

Real and integer field storage ( $x$ for real, $i$ for integer).

real*4 rBDCL1, rBDCL2, rBDCL3, rBDCL4, rBDCL5

integer * 4 iBRND1, iBDAW1, iBRND2 , iBDAW2 , iBRND3 , iBDAW3 , iBRND4 , iBDAW4, - IBRND5, IBDAW5

\section{Nonarrayed maximum field lengths ( 1 for length).}

integer *4 IBSTI, IBCSN, 1BNBSr, IBDCL1, IBDCU1, 1BRND1, 1BRNT1, 1BDAW1, 1BRRC1, 1BASI1, 1BNBSC, 1BDCL2; 1BDCU2, 1BRND2 , 1BRNT2 , IBDAW2, 1BRRC2, IBASI2, IBNBST, IBDCL 3, 1BDCU3, IBRND3, 1BRNT3, 1BDAW3, 1BRRC3, 1BASI3,

- IBNBT, IBDCL4, IBDCU4, IBRND4, IBRNT4, IBDAW4, IBRRC4, IBASI4, IBNBO,

- IBDCL5, IBDCU5, IBNBNi, IBNBFe, IBNBS, IBNBPU, IBRND5, 1BRNT5, IBDAW5,

- IBRRC5, 1BASI5

Nonarrayed null indicators for data transfers (n for null).

integer *2 nBSTI, nBCSN, nBNBSr, nBDCL1, nBDCU1, nBRND1, nBRNT1, nBDAW1, - nBRRC1, nBASI1, nBNBSC, nBDCL2, nBDCU2, nBRND2, nBRNT2, nBDAW2, nBRRC2, - nBASI2, nBNBST, nBDCL3, nBDCU3, nBRND3, nBRNT3, nBDAW3 , nBRRC3 , nBASI3, - nBNBT, nBDCL4 , nBDCO4 , nBRND4 , nBRNT4, nBDAW4 , nBRRC4, nBASI4 , nBNBO,

- nBDCL5, nBDCU5, nBNBNi , nBNBFe, nBNBS, nBNBPu, nBRND5, nBRNT5, nBDAW5,

- nBRRC5, nBASI5

Nonarrayed if-modified indicators (m for modified).

integer*2 mBSTI, mBCSN, mBNBSr, mBDCL1, mBDCU1, mBRND1, mBRNT1, MBDAW1, - mBRRR1, mBASI1, mBNBSC, mBDCL2, mBDCU2 , mBRND2, mBRNT2, mBDAW2, mBRRC2, - mBASI2, mBNBST, mBDCL3, mBDCU3, mBRND3, mBRNT3, mBDAW3, mBRRC3, MBASI3,

- MBNBT, mBDCL4 , MBDCU4 , MBRND4, MBRNT4 , MBDAW4 , MBRRC4 , MBASI4 , mBNBO,

- mBDCL5, mBDCU5, mBNBNi , mBNBFe, mBNBS, mBNBPu, mBRND5, mBRNT5, mBDAW5,

- MBRRC5, mBASI5

\section{Equivalence statements for data transfers.}

equivalence (fidatB(1) ( $1: 12$ ), fBSTI), (ifnulB (1), nBSTI),

- (fidatB (2) $(1: 60)$, fBCSN), (ifnulB (2), nBCSN),

- (fidatB (3) $(1: 1)$, fBNBSr), (ifnulB (3),nBNBSr),

- (fidatB (4) $(1: 4)$, fBDCL1), (ifnulB (4), nBDCL1),

- (fidatB (5) (1:1), fBDCU1), (ifnulB (5), nBDCU1),

- (fidatB $(6)(1: 6)$, fBRND1), (ifnulB (6), nBRND1),

- (fidatB (7) (1:5), fBRNT1), (ifnulB (7), nBRNT1),

- (fidatB (8) $(1: 6)$, fBDAW1), (ifnulB (8), nBDAW1),

- (fidatB (9) $(1: 20)$, fBRRC1), (ifnulB (9), nBRRC1)

- (fidatB $(10)(1: 1)$, fBASI1), (ifnulB (10), nBASII),

- (fidatB (11) $(1: 1)$, fBNBSC), (ifnulB (11), nBNBSC),

- (fidatB (12) (1:4), fBDCL2), (ifnulB (12), nBDCL2),

- (fidatB (13) (1:1), fBDCU2), (ifnulB (13), nBDCU2),

- (fidatB (14) (1:6), fBRND2), (ifnulB (14), nBRND2),

- (fidatB (15) (1:5), fBRNT2), (ifnulB (15), nBRNT2),

- (fidatB $(16)(1: 6)$, fBDAW2), (ifnulB (16), nBDAW2),

- (fidat $B(17)(1: 20)$, fBRRC2), (ifnulB (17), nBRRC2),

- (fidatB (18) (1:1), fBASI2), (ifnulB (18), nBASI2),

- (fidatB (19) (1:1), fBNBST), (ifnulB (19), nBNBST),

- (fidatB $(20)(1: 4)$, fBDCL 3$)$, (ifnulB (20), nBDCL3),

- (fidatB (21) (1:1), fBDCU3), (ifnulB (21), nBDCU3), 
- (fidatB (22) (1:6), fBRND3), (ifnulB (22), nBRND3), - (fidatB (23) (1:5), fBRNT3), (ifnulB (23), nBRNT3), - (fidatB (24) (1:6), fBDAW3), (ifnulB (24), nBDAW3), - (fidatB (25) $(1: 20)$, fBRRC3), (ifnulB (25), nBRRC3), - (fidatB (26) (1:1), fBASI3).. (ifnulB (26), nBASI3),

- (fidatB (27) (1:1), fBNBT), (ifnulB (27), nBNBT),

- (fidatB (28) (1:4), fBDCL4)., (ifnulB (28), nBDCL4),

- (fidatB (29) ( $1: 1)$, fBDCU4), (ifnulB (29), nBDCU4),

- (fidatB $(30)(1: 6)$, fBRND4), (ifnulB (30), nBRND4),

- (fidatB (31) (1:5), fBRNT4), (ifnulB (31), nBRNT4),

- (EidatB (32) (1:6), EBDAW4), (ifnulB (32), nBDAW4),

- (fidatB (33) (1:20), fBRRC4), (i fnulB (33), nBRRC4),

- (fidatB (34) (1:1), fBASI4), (ifnulB (34), nBASI4),

- (fidatB $(35)(1: 1)$, fBNBO), (ifnulB (35), nBNBO),

- (fidatB (36) (1:4), fBDCL5), (ifnulB (36), nBDCL5), ,

- (fidatB (37) (1:1), fBDCU5), (ifnulB (37), nBDCU5),

- (fidatB (38) (1:1), fBNBNi), (ifnulB (38), nBNBNi),

- (fidatB (39) (1:1), EBNBFe), (ifnulB (39), nBNBFe),

- (fidatB $(40)(1: 1)$, fBNBS), (ifnulB $(40), n B N B S)$,

- (fidatB (41) (1:1), fBNBPu), (ifnulB (41), nBNBPu),

- (fidatB (42) (1:6), fBRND5), (ifnulB (42), nBRND5),

- (fidatB (43) (1:5), fBRNT5), (ifnulB (43), nBRNT5),

- (fidatB (44) $(1: 6)$, fBDAW5), (ifnulB (44), nBDAW5),

- (fidatB (45) (1:20), fBRRC5), (ifnulB (45), nBRRC5),

- (fidatB (46) (1:1), fBASI5), (ifnulB (46), nBASI5),

- (mxnocB (1), lBSTI), (ifmodB (1), mBSTI),

- (mXnocB (2), 1BCSN), (i fmodB (2), mBCSN),

- (mxnocB (3), 1BNBSr), (ifmodB (3), mBNBSr),

- (mxnocB (4), IBDCL1), (ifmodB (4), mBDCL1),

$\therefore(\operatorname{mxnocB}(5)$, IBDCU1), (i $\operatorname{mmodB}(5), \operatorname{mBDCU1} 1)$,

- (mxnocB $(6), 1 B R N D 1)$, (ifmodB (6) , mBRND1),

- (mxnocB (7), lBRNT1), (ifmodB (7), mBRNT1),

- (mXnocB (8), IBDAW1), (ifmodB (8), mBDAW1),

- (mXnOCB (9), lBRRC1), (ifmodB (9), mBRRC1),

- (mxnocB (10), IBASI1), (ifmodB (10), mBASI1),

- (mxnocB (11), IBNBSC), (i fmodB (11), mBNBSC),

- (mxnOcB (12), IBDCL2), (ifmodB (12) , mBDCL2),

- (mxnocB (13), 1BDCU2), (i imodB (13) , mBDCU2),

- (mxnOcB (14), IBRND2), (i $\operatorname{lmodB}(14)$, mBRND2),

- (mXnocB (15), IBRNT2), (i $\operatorname{fmodB}(15)$, mBRNT2),

- (mXnOcB (16), IBDAW2), (i $\operatorname{modB}(16)$, MBDAW2),

- (mXnOCB (17), IBRRC2), (i $\operatorname{modB}(17)$, MBRRC2),

- (mXnOcB (18), IBASI2), (ifmodB (18), mBASI2),

- (mXnOCB (19); IBNBST), (ifmodB (19), MBNBST),

- (mxnOcB (20), 1BDCL3), (ifmodB (20), mBDCL3),

- (mXnOcB (21) , IBDCU3), (i $\operatorname{modB}(21)$, mBDCU3),

- (mXnOcB (22), 1BRND3), (ifmodB (22), mBRND3),

- (mXnOCB (23), 1BRNT3), (i $\operatorname{modB}(23), \operatorname{mBRNT3})$,

- (mXnOcB (24), 1BDAW3), (i $\operatorname{mmodB}(24), \operatorname{mBDAW} 3)$,

- (mXnOCB (25), 1BRRC3), (i $\operatorname{lmodB}(25)$, mBRRC3),

- (mxnOcB (26), 1BASI3), (ifmodB (26), mBASI3),

- (mxnocB (27), lBNBT), (ifmodB (27), mBNBT),

- (mxnocB $(28), 1 B D C L 4)$, (i $\operatorname{modB}(28), \operatorname{mBDCL} 4)$,

- (mxnocB (29), 1BDCU4), (i $\operatorname{modB}(29), \operatorname{mBDCU4})$,

- (mxnocB (30), 1BRND4), (i $\operatorname{fmodB}(30), \operatorname{mBRND} 4)$,

- (mXnOcB (31), 1BRNT4), (i $\operatorname{mmodB}(31), \operatorname{mBRNT4}$ ),

- (mxnOcB (32), 1BDAW4), (ifmodB (32), mBDAW4),

- (mxnocB (33), IBRRC4), (i $\operatorname{imodB}(33), \operatorname{mBRRC4})$,

- (mxnocB (34), IBASI4), (i $\operatorname{modB}(34)$, mBASI4),

- (mxnocB $(35), 1 \mathrm{BNBO}),($ ifmodB (35), mBNBO), 
- (mxnocB (36), 1BDCL5), (ifmodB (36), mBDCL5),

- (mxnocB (37), 1BDCU5), (i $\operatorname{lmodB}(37)$, mBDCU5),

- (mxnocB (38), IBNBNi), (i $\operatorname{modB}(38), \operatorname{mBNBNi})$,

- (mxnocB (39), lBNBFe), (i fmodB (39), mBNBFe),

- (mxriocB $(40)$, IBNBS), (ifmodB (40), mBNBS),

- (mXnOcB (41), IBNBPu), (i $\operatorname{modB}(41), \operatorname{mBNBPu})$,

- (mXnOcB (42), IBRND5), (i $\operatorname{modB}(42)$, mBRND5),

- (mXnOcB (43), IBRNT5), (i $\bmod$ ( 143$)$, mBRNT5),

- (mxnOcB $(44)$, IBDAW5), (i $\operatorname{modB}(44)$, mBDAW5),

- (mxnOcB (45), 1BRRC5), (i imodB (45), mBRRC5),

- (mxnocB $(46), 1$ BASI5), (ifmodB (46), mBASI5)

Database table and attribute names for screen fields.

data atrbtB/

- 'BETA_STRONTIUM_90','SAMPLE_TRACKING'ID',

'SAMPLE_INFORMATION' , 'CUSTOMER_SAMPLE_NAME',

- 'SAMPLE_INFORMATION' ,'NEED_BETA_STRONTIUM_90',

- 'BETA_STRONTIUM_90', 'DETECTOR_COUNT_LENGTH',

'BETA_STRONTIUM_90','DETECTOR_COUNT_LENGTH_UNITS',

'BETA_STRONTIUM_90', 'RESULTS_NNEEDED_BY_DATE',

'BETA_STRONTIUM_90','RESULTS_NEEDED_BY_TIME',

'BETA_STRONTIUM_90', 'DATE_ALI_WORK_COMPLETED',

'BETA_STRONTIUM_90','RESULTS_REPORT_CITATION',

'BETA_STRONTIUM_90', 'ANY_SPECIAI_INSTRUCTIONS',

' SAMPLE_INFORMATION' , NEED_BETA_STRONTIUM_89_AND_90',

'BETA_STRONTIUM_89_AND_90','DETECTOR_COUNT_IENGTH',

'BETA_STRONTIUM_89_AND_90', 'DETECTOR_COUNT_IENGTH_UNITS' ,

'BETA_STRONTIUM_89_AND_90','RESULTS_NEEDED_BY_DATE',

'BETA_STRONTIUM_89_AND_90', 'RESULTS_NEEDED_BY_TIME',

'BETA_STRONTIUM_89_AND_90', 'DATE_ALI_WORK_COMPLETED',

'BETA_STRONTIUM_89_AND_90', 'RESULTS_REPORT_CITATION',

'BETA_STRONTIUM_89_AND_90',' ANY_SPECIAL_INSTRUCTIONS',

'SAMPLE_INFORMATION' , 'NEED_BETA_TOTAL_STRONTIUM',

'BETA_TOTAL_STRONTIUM' , 'DETECTOR_COUNT_LENGTH',

'BETA_TOTAL_STRONTIUM', 'DETECTOR_COUNT_LENGTH_UNITS',

'BETA_TOTAL_STRONTIUM','RESULTS_NEEDED_EY_DATE',

'BETA_TOTAL_STRONTIUM', 'RESULTS_NEEDED_EY_TIME',

'BETA_TOTAL_STRONTIUM', 'DATE_AIL_WORK_COMPLETED',

'BETA_TOTAL_STRONTIUM', 'RESULTS_REPORT_CITATION',

- 'BETA_TOTAL_STRONTIUM', 'ANY_SPECIAL_INSTRUCTIONS',

'SAMPLE_INFORMATION' , 'NEED_BETA_TRITIUM',

'BETA_TRITIUM', 'DETECTOR_COUNT_LENGTH',

'BETA_TRITIUM','DETECTOR_COUNT_LENGTH_UNITS',

'BETA_TRITIUM','RESULTS_NEEDED_BY_DATE',

'BETA_TRITIUM', 'RESULTS_NEEDED_BY_TIME',

'BETA_TRITIUM','DATE_ALI_WORK_COMPLETED',

'BETA_TRITIUM',' RESULTS_REPORT_CITATION',

- 'BETA_TRITIUM', 'ANY_SPECIAI_INSTRUCTIONS',

'SAMPLE_INFORMATION',' NEED_BETA_OTHER',

'BETA_OTHER', 'DETECTOR_COUNT_LENGTH',

'BETA_OTHER', 'DETECTOR_COUNT_LENGTH_UNITS' ,

'BETA_OTHER', 'NEED_BETA_NICKEL_63',

'BETA_OTHER', 'NEED_BETA_IRON_55',

'BETA_OTHER', 'NEED_BETA_SULFUR_3.5',

'BETA_OTHER', 'NEED_BETA_PLUTONIUM_241',

'BETA_OTHER', 'RESULTS_NEEDED_BY_DATTE',

'BETA_OTHER', 'RESULTS_NEEDED_BY_TIME',

- 'BETA_OTHER', 'DATE_ALE_WORK_COMPIETED',

- 'BETA_OTHER',' RESUITS_REPORT_CITATION', 
C

C

C

C
C
C

C

C

C

$\mathrm{C}$

C

. 'BETA_OTHER' , 'ANY_SPECIAL_INSTRUCTIONS' /

Program variable names (value and null indicator) for screen fields.

data varblB/':fBSTI:nBSTI' ', :EBCSN:nBCSN' , : fBNBSr:nBNBSr',

. ' : rBDCL1: nBDCL1',' : fBDCU1:nBDCU1', ': iBRND1 :nBRND1',

' : fBRNT1:nBRNT1', ' : iBDAW1:nBDAW1',' : IBRRC1:nBRRC1',

$'$ : fBASI1 : nBASI1',' : fBNBSC:nBNBSC', ' : rBDCL2 : nBDCL2',

' : fBDCU2 : nBDCU2', ' : iBRND2 :nBRND2', ' : fBRNT2 : nBRNT2',

' : IBDAW2 : nBDAW2', ' : fBRRC2 : nBRRC2', ' : fBASI2 :nBASI2',

' : fBNBST: nBNBST', ' : rBDCL3 :nBDCL3', ' : EBDCU3 :nBDCU3',

' : iBRND3 :nBRND3' , : IBRNT3 :nBRNT3', ' : iBDAW3 :nBDAW3',

' : fBRRC3 :nBRRC 3', ' : fBASI3 :nBASI3', ' : EBNBT: nBNBT',

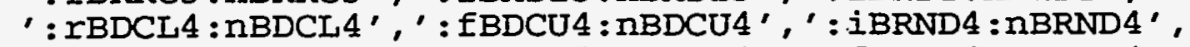

' : fBRNT4 : nBRNT4',' : iBDAW4 : nBDAW4', ' : fBRRC4 :nBRRC4!',

' : fBASI4 :nBASI4', ' : fBNBO:nBNBO' , ' : rBDCL5 :nBDCL5',

' : fBDCU5 :nBDCU5' ,' : fBNBNi :nBNBNi' ' : fBNBFe:nBNBFe',

$'$ : fBNBS:nBNBS', : fBNBPu:nBNBPu' , : iBRND5:nBRND5',

' : fBRNT5 :nBRNT5', ' : iBDAW5 : nBDAW5' , ' : fBRRC5 : nBRRC5',

' : fBASI5 :nBASI5'/

Gamma screen's field descriptors and field sizes.

character $* 80$ fieldG (35), fidatg (35)

character $\star 30$ atrbtg $(2,35)$

character $* 14$ varblG (35)

character*1 fitypg(35)

integer*4 faleng (35), farowG (35), fdcolg (35), ficolg (35), mxnocG (35),

- fdrndG $(3,35)$, firndG $(3,35)$, ! Field renditions (add/update/search).

- numfaG/35/ !The number of fields.

integer*2 ifnulg(35), !Null indicator (-1 if null, 0 otherwise).

- ifrqdG (35) $/ 35 * 0 /$, !points to if-required dependent field.

- iditoG.(35)/35*0/ ! Points to allowed field for dittoing.

logical ifmodG(35) ! Has a field been modified in an Update?

common /dbdatG/ fieldG, fidatG, fdleng, fdrowg, fdcolG, ficolg, mxnocG,

- fdrndG, firndG, numfdG, fitypG, ifnulG, atrbtG, varblG, ifrqdG, ifmodG,

- iditog

Length-specified character fields for equivalences ( $f$ for field).

character fGSTI*12, fGCSN*60, fGNGS*1, fGDCL1*4, fGDCU1*1, fGRND1*6,

. fGRNT $1 * 5$, fGDAW $1 * 6$, fGDSC $1 * 6$, fGRSI $1 * 14$, fGITA $1 * 1$, fGRRC $1 * 20$, fGASI $1 * 1$,

- fGNGFI* 1 , fGDCL 2 * 4 ; fGDCU2 21 , fGRND 2 * 6 , fGRNT2 * 5, fGDAW2 * 6 , fGDSC2 * 6 ,

- fGRSI $2 * 14$, fGITA $2 * 1$, fGRRC2 220, fGASI 2 * 1 , fGNGO 1 , fGDCL $3 * 4$, fGDCU $3 * 1$,

- fGRND $3 * 6$, fGRNT3 $3 * 5$, fGDAW $3 * 6$, fGDSC $3 * 6$, fGRSI $3 * 14$, fGITA $3 * 1$, fGRRC $3 * 20$,

- fGASI $3 * 1$

Real and integer field storage (r for real, $i$ for integer).

real*4 rGDCL1, rGDCL 2, rGDCL 3

integer 4 iGRND1, iGDAW1, iGDSC1, iGRND2 , iGDAW2 , iGDSC2, iGRND3 , iGDAW3, - iGDSC3

Nonarrayed maximum field lengths (l for length).

integer *4 1GSTI, 1GCSN, 1GNGS, 1GDCL1, 1GDCU1, 1GRND1, 1GRNT1, IGDAW1, - IGDSC1, IGRSI1, IGITA1, IGRRC1, IGASI1, IGNGFI, IGDCL2, IGDCU2, IGRND2,

- IGRNT2, I GDAW2, I GDSC2, I GRSI2, IGITA2, IGRRC2, IGASI2, IGNGO, I GDCL 3 ,

- IGDCU3, IGRND3, 1GRNT3, I GDAW3, IGDSC3, IGRSI3, IGITA3, IGRRC3, IGASI3 
integer *2 nGSTI, nGCSN, nGNGS, nGDCL1, nGDCU1, nGRND1, nGRNT1, nGDAW1, - nGDSC1, nGRSI1, nGITA1, nGRRC1, nGASI1, nGNGFI, nGDCI2, nGDCU2, nGRND2, - nGRNT2, nGDAW2, nGDSC2, nGRSI2, nGITA2, nGRRC2, nGASI2, nGNGO, nGDCL3,

- nGDCU3, nGRND3, nGRNT3, nGDAW3, nGDSC3, nGRSI3, nGITA3, nGRRC3, nGASI3

$$
\text { Nonarrayed if-modified indicators ( } m \text { for modified). }
$$

integer *2 mGSTI, mGCSN, MGNGS, MGDCL1, mGDCU1, mGRND1, mGRNT1, MGDAW1,

- MGDSC1, MGRSI1, mGITA1, MGRRC1, MGASI1 , MGNGFI, MGDCL2 , MGDCU2 , MGRND2,

- MGRNT2 , MGDAW2, MGDSC2, mGRSI2, MGITA2 , MGRRC2, MGASI2, mGNGO, MGDCL3,

C

mGDCU3, mGRND3, mGRNT3, mGDAW3, mGDSC 3, mGRSI3, mGITA3, mGRRC3, MGASI3

C

\section{Equivalence statements: for data transfers.}

equivalence (fidatg(1) (1:12), fGSTI), (ifnulG(1), nGSTI),

(fidatg(2) $(1: 60)$, fGCSN), (ifnulg(2), nGCSN),

- (fidatg (3) (1:1), fGNGS), (ifnulg (3), nGNGS),

- (fidatG(4) (1:4), fGDCL1), (ifnulG(4), nGDCL1),

- (fidatG (5) (1:1), fGDCU1), (ifnulg (5), nGDCU1),

- (EidatG $(6)(1: 6)$, fGRND1), (ifnulg $(6)$, nGRND1),

- (fidatG (7) (1:5), fGRNT1), (ifnulg(7), nGRNT1),

- (fidatG(8) $(1: 6)$, fGDAWI), (ifnulg(8), nGDAW1),

- (fidatG (9) (1:6), fGDSC1), (ifnulg(9), nGDSC1),

- (fidatg(10) (1:14), fGRSI1), (ifnulg(10), nGRSI1),

- (fidatG(11) (1:1), fGITA1), (ifnulg (11), nGITA1),

- (fidatg(12) (1:20), fGRRC1), (ifnulg(12), nGRRCI),

- (fidatg(13) (1:1), fGASI1), (ifnulg(13), nGASI1),

- (fidatg (14) (1:1), fGNGFI), (ifnulg (14), nGNGFI),

- (fidatG(15) (1:4), fGDCL2), (ifnulg(15), nGDCL2),

- (fidatG(16) (1:1), fGDCU2), (ifnulg(16), nGDCU2),

- (fidatg(17) (1:6), fGRND2), (ifnulg (17), nGRND2),

- (fidatG (18) (1:5), fGRNT2), (ifnulg (18), nGRNT2),

- (fidatg (19) (1:6), fGDAW2), (ifnulg(19), nGDAW2),

- (fidatg(20) (1:6), fGDSC2), (ifnulg(20), nGDSC2),

- (fidatg(21) (1:14), fGRSI2), (ifnulg(21), nGRSI2),

- (fidatG(22) (1:1), fGITA2), (ifnulg(22), nGITA2),

- (fidatg (23) (1:20), fGRRC2), (ifnulg(23), nGRRC2),

(fidatg(24) (1:1), fGASI2), (ifnulg(24), nGASI2),

- (fidatG(25) (1:1), fGNGO), (ifnulg(25), nGNGO),

- (fidatg (26) (1:4), fGDCL3), (ifnulg (26), nGDCL3),

- (fidatg(27) (1:1), fGDCU3), (ifnulg (27), nGDCU3),

- (fidatg (28) (1:6), fGRND3), (ifnulg (28), nGRND3),

- (fidatg (29) (1:5), fGRNT3), (i inulg (29), nGRNT3),

- (fidatG $(30)(1: 6)$, fGDAw3), (ifnulg (30), nGDAW3),

- (fidatg (31) (1:6), fGDSC3), (ifnulg (31), nGDSC3),

- (fidatg (32) (1:14), fGRSI3), (ifnulg(32), nGRSI3),

- (fidatg (33) (1:1), fGITA3), (ifnulg (33), nGITA3),

- (fidatg (34) (1:20), fGRRC3), (i fnulg (34), nGRRC3),

- (fidatg (35) (1:1), fGASI3), (ifnulg (35), nGASI3),

- (mXnocG (1), IGSTI), (ifmodG (1), MGSTI),

- (mXnOcG (2), IGCSN), (i imodg (2), mGCSN),

- (mXnocG (3), IGNGS), (i fmodG (3), mGNGS),

- (mXnocG (4), IGDCL1), (ifmodG (4), MGDCL1),

- (mXnocG (5), IGDCUI) : (ifmodG (5), mGDCUI),

- (mXnOCG (6), I GRNDI), (i

- (mXnocG (7), 1GRNT1), (ifmodG (7), mGRNT1),

- (mXnocG (8), I GDAW1), (ifmodG (8), mGDAW1),

- (mXnocG (9), IGDSC1), (ifmodG (9), mGDSC1), 
- (mxnocG (10), IGRSI1), (ifmodG(10), mGRSI1), - (mXnocG (11), IGITA1), (ifmodG (11), mGITA1), - (mXnOcG (12), IGRRC1), (ifmodG (12), mGRRC1), - (mxnocG (13), I GASI1), (ifmodG (13), MGASI1), - (mXnocG (14), IGNGFI), (ifmodG'(14)',mGNGFI), - (mxnocG (15), 1GDCL2) , (ifmodG (15), mGDCL2), - (mXnocG (16), IGDCU2), (ifmodG (16), MGDCU2)., - (mxnocG (17), IGRND2), (i imodG (17), MGRND2) , - (mXnOCG (18), IGRNT2), (i $\operatorname{fmodG}(18), \operatorname{mGRNT2}$ ), - (mxnocG (19), IGDAW2), (ifmodG (19), mGDAW2), . (mxnocG $(20), 1$ GDSC2 $)$, (i $\operatorname{fmodg}(20), \operatorname{mGDSC} 2)$, - (mxnocG (21), 1GRSI2), (ifmodG (21), mGRSI2), - (mxnocG (22), IGITA2), (i fmodG (22), mGITA2), - (mxnocG (23), IGRRC2) , (i fmodg (23), mGRRC2), - (mXnocG (24), 1GASI2), (ifmodG (24), mGASI2),

- (mxnocG (25), IGNGO), (ifmodG (25), mGNGO),

- (mxnocG $(26), 1$ GDCL 3$)$, (ifmodG (26), mGDCL3),

- (mxnocG (27), IGDCU3), (ifmodG (27), mGDCU3),

- (mXnOcG (28), IGRND3), (i imodG (28), mGRND3),

- (mxnocG (29), IGRNT3), (ifmodG (29), mGRNT3),

- (mxnocG (30), I GDAW3), (i imodG (30), mGDAW3),

- (mxnocG (31), 1GDSC3), (ifmodG (31), mGDSC3),

- (mXnocG (32), IGRSI3), (ifmodG (32), mGRSI3),

- (mxnocG (33), IGITA3), (ifmodg (33), mGITA3),

- (mXnocG (34), IGRRC3), (ifmodG (34), mGRRC3),

- (mxnocG (35), IGASI3), (ímodG (35), mGASI3)

C

$C$

Database table and attribute names for screen fields.

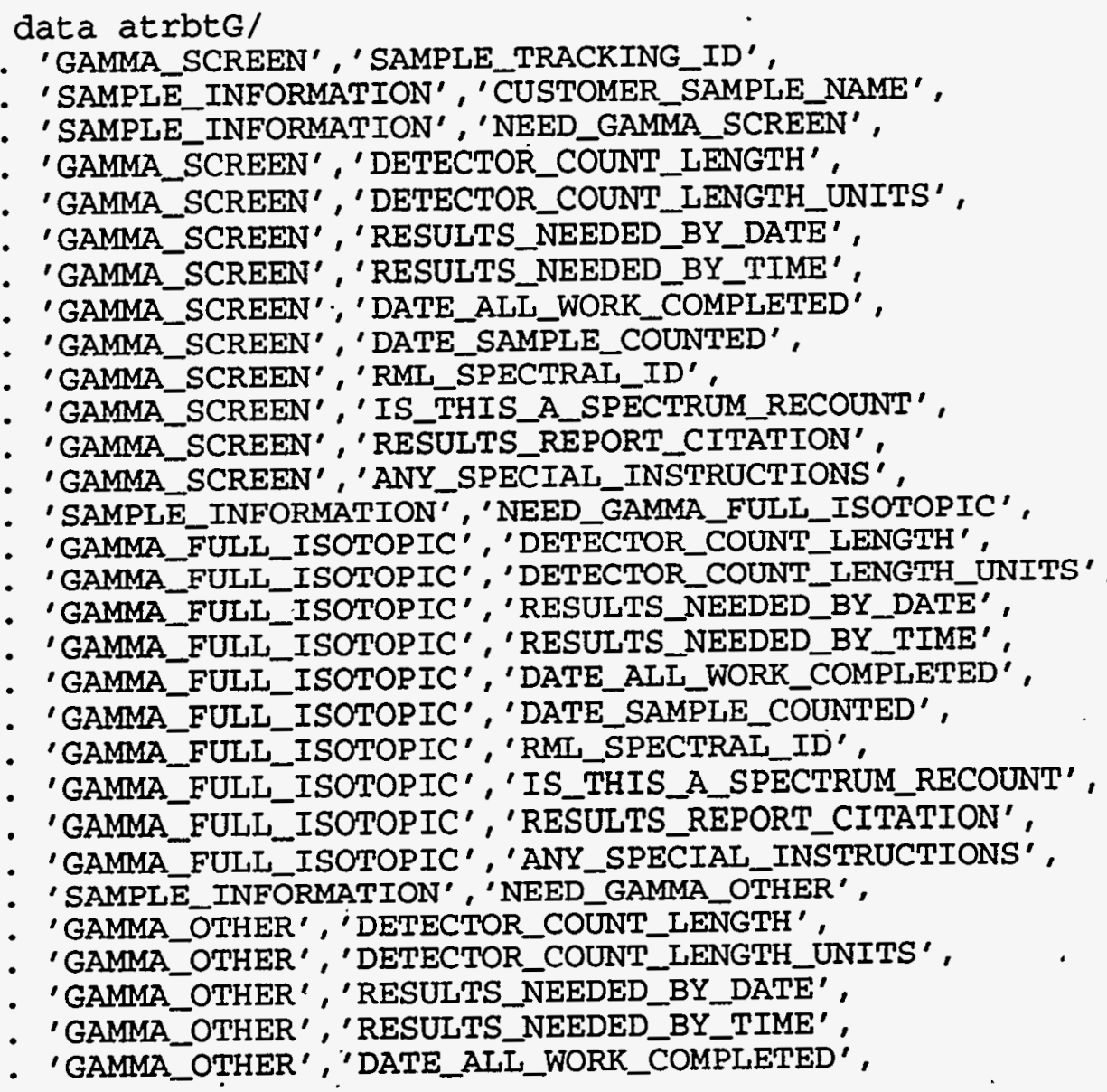




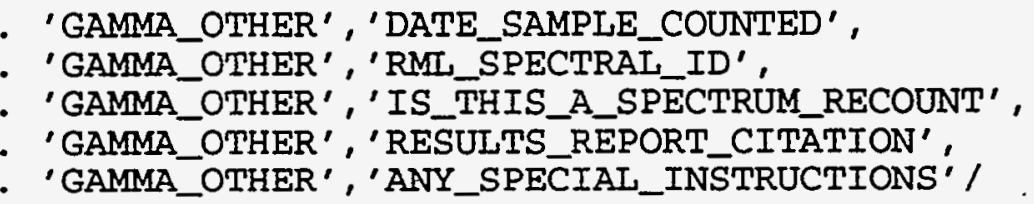

C

$\mathrm{C}$
$\mathrm{C}$

C

C

C

$\mathrm{C}$
$\mathrm{C}$
$\mathrm{C}$

$\mathrm{C}$

C

C

C

C

C

Program variable names (value and null indicator) for screen fields.

data varblG/' : fGSTI:nGSTI', ' : fGCSN:nGCSN' ,' : fGNGS:nGNGS',

. ': rGDCL1: nGDCL1' ' : fGDCU1: nGDCU1', ': iGRND1 : nGRND1',

' ' : fGRNT1 : nGRNT1',' : iGDAW1 : nGDAW1', ': iGDSC1 :nGDSC1',

' : fGRSI1 : nGRSI1', ' : fGITA1 : nGITA1', ' : fGRRC1 : nGRRC1',

' : IGGASI1 : nGASI1', ' : fGNGFI : nGNGFI' ' : IGGCL2 : nGDCL2',

' : fGDCU2 : nGDCU2', ' : iGRND2 : nGRND2', ' : fGRNT2 : nGRNT2',

' : iGDAW2 : nGDAW2', ' : iGDSC2 : nGDSC2', ' : fGRSI2 : nGRSI2',

' : fGITA2 : nGITA2' ' : fGRRC2 : nGRRC2 ${ }^{\prime}$, ' : fGASI2 : nGASI2',

' : fGNGO: nGNGO' , ' : rGDCL 3 : nGDCL $3^{\prime}$, ' $^{\prime}$ : IGDCU3 : nGDCU3',

' : iGRND3 : nGRND3' ' ' : fGRNT3 : nGRNT3', ' : iGDAW3 : nGDAW3',

- ': iGDSC3 :nGDSC3',' : fGRSI3:nGRSI3', ' : fGITA3 :nGITA3',

. ' : fGRRC3 : nGRRC3!', ' : fGASI3:nGASI3',

Gross alpha-beta screen's field descriptors and field sizes.

character $* 80$ fieldC (18), fidatc(18)

character $* 30$ atrbtc $(2,18)$

character $* 14$ varblc (18)

character*1 fitypc(18)

integer*4 fdlenc (18), farowC (18), fdcolc(18), ficolc (18), mxnocc (18),

. fdrndc $(3,18)$; firndC $(3,18)$, ! Field renditions (add/update/search).

- numfdC/18/ !The number of fields.

integer 2 ifnulC(18), !Null indicator (-1 if null, 0 otherwise).

- ifrqdC (18)/18*0\%, ! points to if-required dependent field.

- iditoc $(18) / 18 * 0 /$ ! points to allowed field for dittoing.

logical ifmodC(18) !Has a field been modified in an Update?

common /dbdatc/ fieldC, fidatc, fdlenc, fdrowc, fdcolc, ficolc, mxnocC,

- fdrndC, firndC, numfdC, fitypc, ifnulc, atrbtc, varblc, ifrqdC, ifmodC,

- iditoC

Length-specified character fields for equivalences ( 1 for length).

character fCSTI *12, fCCSN*60, fCNGAF*1, fCDCL $1 * 4, f C D C U 1 * 1, f C R N D 1 * 6$,

- fCRNT1 15, fCDAW 1 *6, fCRRC $1 * 20$, fCASI $1 * 1$, fCNGAO $* 1$, fCDCL $2 * 4$, fCDCU 2 * 1 ,

- $\mathrm{fCRND} 2 * 6$, fCRNT2 25, fCDAW $2 * 6$, fCRRC $2 * 20$, fCASI $2 * 1$

Real and integer field storage ( $r$ for real, i for integer).

real* 4 rCDCL1, rCDCL2

integer * 4 iCRND1, iCDAW1, iCRND2, iCDAW2

Nonarrayed maximum field lengths ( 1 for length) :

integer *4 1CSTI, 1CCSN, 1CNGAF, 1CDCL1, 1CDCU1, 1CRND1, 1CRNT1, 1CDAW1, - ICRRC1, ICASI1, I CNGAO, I CDCL2, ICDCU2, ICRND2, ICRNT2, ICDAW2, ICRRC2,

- ICASI2

Nonarrayed null indicators for data transfers (n for null).

integer * 2 nCSTI, nCCSN, nCNGAF, nCDCL1, nCDCU1, nCRND1, nCRNT1, nCDAW1, - nCRRC1, nCASI1, nCNGAO, nCDCL2, nCDCU2, nCRND2, nCRNT2, nCDAW2, nCRRC2, - nCASI2 


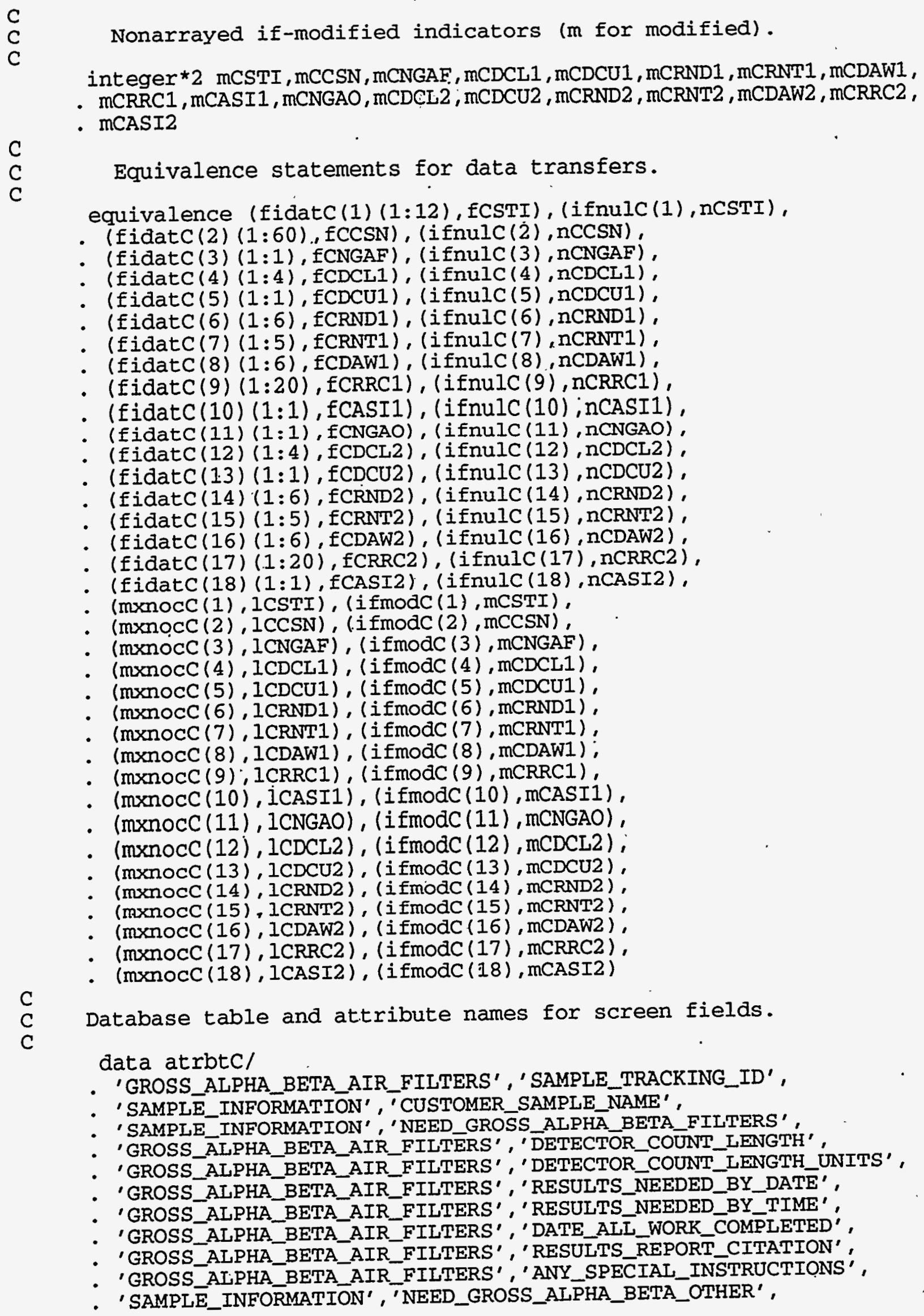


- 'GROSS_ALPHA_BETA_OTHER', 'DETECTOR_COUNT_LENGTH',

'GROSS_ALPHA_BETA_OTHER', 'DETECTOR_COUNT_LENGTH_UNITS',

- 'GROSS_ALPHA_BETA_OTHER', 'RESULTS_NNEEDED_BY_DATE',

'GROSS_ALPHA_BETA_OTHER', 'RESULTS_NEEDED_BY_TIME',

'GROSS_ALPHA_BETA_OTHER', 'DATE_ALI_WORK_COMPLETED' ,

'GROSS_ALPHA_BETA_OTHER', 'RESULTS_REPORT_CITATION',

- 'GROSS_ALPHA_BETA_OTHER', 'ANY_SPECIAL_INSTRUCTIONS'

C

C

C

C

$\mathrm{C}$

C

C

C

C
C
C
C
C
C
C

$\mathrm{C}$
$\mathrm{C}$

Program variable names (value and null indicator) for screen fields.

data varblC/': fCSTI:nCSTI',':fCCSN:nCCSN',' :fCNGAF:nCNGAF',

- ' : rCDCL1 : nCDCL1', ' fCDCU1 : nCDCU1',': iCRND1 : nCRND1',

. ' : fCRNT1 : nCRNT1',' : iCDAW1 : nCDAW1', ' : fCRRC1 : nCRRC1',

. ' : fCASI1 : nCASI1',' : fCNGAO: nCNGAO', ' : rCDCL2 : nCDCL2',

. ' : fCDCU2 : nCDCU2', ' : iCRND2 : nCRND2', ' : fCRNT2 : nCRNT2',

. ' : iCDAW2 : nCDAW2', ' :.fCRRC2 : nCRRC2', ' : fCASI2 :nCASI2'

Special instruction screen's field descriptors and field sizes.

character*80 fieldI(32), fidatI(32)

character $* 30$ atrbtI $(2,5)$

character $* 14$ varblI (5)

character*1 fitypI (32)

integer* 4 fdlenI(32), fdrowI (32), fdcolI (32), ficolI (32), mxnocI (32),

- farnaI $(3,32)$, firndI $(3,32)$, ! Field renditions (add/update/search).

- numfdI/32/ !The number of fields.

integer*2 ifnulI(32), !Null indicator (-1 if null, 0 otherwise).

- ifrqdI $(32) / 32 * 0 \%$ ! Points to if-required dependent field.

- iditoI (32)/32*0/ ! Points to allowed field for dittoing.

logical ifmodI(32) !Has a field been modified in an Update?

common /dbdatI/ fieldI, fidatI, fdlenI, fdrowI, fdcolI, ficolI,mxnocI,

- fdrndI, firndI, numfdI, fitypI, ifnulI, atrbt I, varblI, ifrqdI, ifmodI,

- iditoI

Length-specified character fields for equivalences (f for field).

character fISTI*12, fICSN*60, fIOS $1 * 16$, fISI $1 * 50, \mathrm{fIOS} 2 * 16, \mathrm{fISI} 2 * 50$,

- fIOS $3 * 16, \mathrm{fISI} 3 * 50, \mathrm{fIOS} 4 * 16, \mathrm{fISI} 4 * 50, \mathrm{fIOS} 5 * 16, \mathrm{fISI5} * 50, \mathrm{fIOS} 6 * 16$,

- fISI6*50, fIOS $7 * 16$, fISI $7 * 50$, fIOS $8 * 16$, fISI $8 * 50$, fIOS $9 * 16$, fISIg *50,

- fIOS $10 * 16$, fISI $10 * 50$, fIOS $11 * 16$, fISI $11 * 50$, fIOS $12 * 16$, fISII $12 * 50$,

- fIOS13*16, fISI $13 * 50$, fIOS $14 * 16$, fISI $14 * 50$, fIOS15*16, fISII5*50,

- fIOS1t*16,fISI $1 t * 50$ ! And spares for inserts and fetchs.

Integer field storage ( $i$ for integer).

integer*4 siline !Declared in the main routine.

Nonarrayed maximum field lengths ( 1 for length).

integer *4 IISTI, IICSN, IIOS1, IISI1, 1IOS2, IISI2, 1IOS3, 1ISI3, 1IOS4,

- 1ISI4, 1IOS5, 1ISI5, 1IOS6, IISI6, 1IOS7, IISI7, IIOS8, 1ISI8, 1IOS9,

- IISI9, IIOS10, IISI10, IIOS11, IISI11, IIOS12, IISI12, 1IOS13, IISI13,

- IIOS14, IISI14, IIOS15, IISI15,

- lIOS1t, IISI1t !And spares for inserts and fetchs.

Nonarrayed null icators for data transfers (n for null).

integer*2 nISTI, nIC SN, nIOS1, nISI1, nIOS2, nISI2, nIOS3, nISI3, nIOS4,

- nISI4, nIOS5, nISI5, nIOS6, nISI6, nIOS7, nISI7, nIOS8, nISI8, nIOS9,

- nISI9, nIOS10,nISI10, nIOS11, nISI11, nIOS12, nISI12, nIOS13, n:ISI13, 
- nIOS14,nISI14,nIOS15;nISI15,

- nIOSIt,nISI1t !And spares for inserts and fetchs.

$\mathrm{C}$
$\mathrm{C}$
$\mathrm{C}$

Nonarrayed if-modified indicators (m for modified).

integer *2 mISTI, mICSN, mIOS1, mISI1, mIOS2, mISI2, mIOS3, mISI3, mIOS4,

- mISI4, mIOS5, mISI5, mIOS6, mISI6, mIOS7, mISI7, mIOS8, mISI8, mIOS9,

- mISI9,mIOS10,mISI10,mIOS11,mISI11,mIOS12,mISI12,mIOS13,mISI13,

- mIOS14,mISI14,mIOS15,mISI15,

$\mathrm{C}$
$\mathrm{C}$
$\mathrm{C}$

- mIOS1t,mISI1t !And spares. for inserts and fetchs.

Equivalence statements for data transfers.

equivalence (fidatI(1) (1:12), fISTI), (ifnulI(1),nISTI),

(fidatI (2) (1:60), fICSN), (ifnulI (2), nICSN),

(fidatI (3) (1:16), EIOSI),(ifnulI(3),nIOS1),

- (fidatI (4) (1:50), EISI1), (ifnulI (4),nISI1),

- (fidatI (5) (1:16), fIOS2), (ifnulI(5), nIOS2),

- (EidatI (6) $(1: 50)$, fISI2), (ifnulI (6), nISI2),

- (fidatI (7) (1:16), fIOS3), (ifnulI(7),nIOS3),

- (fidatI (8) (1:50), fISI3), (ifnulI(8),nISI3),

- (fidatI (9) (1:16), fIOS4), (ifnulI (9), nIOS4),

- (EidatI (10) (1:50), fISI4), (ifnulI(10),nISI4),

- (EidatI (11) (1:16), fIOS5), (ifnulI (11), nIOS5),

- (fidatI (12) (1:50), fISI5), (ifnulI(12),nISI5),

- (fidatI (13) (1:16), fIOS6), (ifnulI (13),nIOS6),

- (EidatI (14) (1:50), fISI6), (ifnulI (14),nISI6),

- (fidatI (15) (1:16), fIOS7), (ifnulI (15),nIOS7),

- (fidatI(16) (1:50), fISI7), (ifnulI(16),nISI7),

- (fidatI (17) (1:16), fIOS8), (ifnulI (17),nIOS8),

- (fidatI(18) (1:50), fISI8), (ifnulI (18),nISI8),

- (EidatI (19) (1:16), fIOS9), (ifnulI (19),nIOS9),

- (fidatI(20) (1:50), fISI9), (ifnulI (20),nISI9),

- (fidatI (21) (1:16), fIOS10), (ifnulI(21),nIOS10),

- (fidatI (22) (1:50), fISI10), (ifnulI (22),nISI10),

- (fidatI (23) (1:16), fIOS11), (ifnulI (23), nIOS11),

- (fidatI (24) (1:50), fISI11), (ifnulI (24),nISI11),

- (fidatI (25) (1:16), fIOS12), (ifnulI (25),nIOS12),

- (fidatI (26) (1:50), fISI12), (ifnulI(26),nISI12),

- (fidatI (27) (1:16), fIOS13), (ifnulI (27), nIOS13),

- (EidatI(28) (1:50), fISI13), (i fnulI (28),nISI13),

- (fidatI(29) (1:16), fIOS14), (ifnulI(29),nIOS14),

- (fidatI (30) (1:50), fISI14), (ifnulI (30), nISI14),

- (fidatI(31) (1:16), fIOS15), (ifnulI(31),nIOS15),

- (fidatI (32) (1:50), fISI15), (ifnulI (32),nISI15),

- (mXnocI (1), IISTI), (ifmodI (1), MISTI),

- (mXnocI (2), IICSN), (ifmodI (2), mICSN),

- (mxnocI (3), IIOS1), (ifmodI (3), mIOSI),

- (mxnocI (4), IISI1), (ifmodI (4), mISII).

- (mxnocI (5), IIOS2), (ifmodI (5), mIOS2),

- (mxnocI (6), IISI2), (ifmodI (6), mISI2),

- (mxnocr $(7), 1$ IOS 3$)$, (ifmodI (7), mIOS3). (mxnocI (8), IISI3), (i fmodI (8), mISI3),

(mXnocI (9), 1IOS4), (ifmodI (9), mIOS4),

(mxnocI (10), 1ISI4), (ifmodI (10), mISI4),

(mxnocI (11), IIOS5), (ifmodI (11), mIOS5),

(mxnocI (12), IISI5), (ifmodI (12), mISI5),

- (mxnocI (13), IIOS6), (ifmodI (13), mIOS6),

- (mxnocI (14), 1ISI6), (ifmodI (14), mISI6),

- (mxnocI (15), IIOS7), (ifmodI (15), mIOS7), 
- (mxnocI (16), 1ISI7), (ifmodI (16), mISI7),

- (mxnocI (17), IIOS8), (ifmodI (17), mIOS8),

- (mxnocI (18), IISI8), (ifmodI (18), mISI8),

- (mxnocI (19), lIOS9), (ifmodI (19), mIOS9),

- (mxnocI (20), IISI9), (ifmodI (20), mISI9),

- (mxnocI (21), IIOS10), (ifmodI (21), mIOS10),

- (mxnocI (22), IISI10), (ifmodI (22), mISI10),

- (mxnocI (23), IIOS11), (ifmodI (23), mIOS11),

- (mxnocI (24), IISI11), (ifmodI (24), mISI11),

- (mxnocI (25), IIOS12), (ifmodI (25), mIOS12),

- (mxnocI (26), IISI12), (ifmodI (26), mISI12),

- (mxnocI (27), IIOS13), (ifmodI (27), mIOS13),

- (mxnocI (28), IISI13), (ifmodI (28), mISI13),

- (mxnocI (29), IIOS14), (ifmodI (29), mIOS14),

- (mxnocI (30), IISI14), (ifmodI (30), mISI14),

- (mxnocI (31), IIOS15), (ifmodI (31), mIOS15),

- (mxnocI (32), IISI15), (ifmodI (32), mISI15)

C

$\mathrm{C}$

Database table and attribute names for screen fields.

data atrbtI/

'SPECIAL_INSTRUCTIONS', 'SAMPLE_TRACKING_ID',

- 'SAMPLE_INFORMATION' ' CUSTOMER_SAMPLE_NAME',

' 'SPECIAI_INSTRUCTIONS','ORIGIN_OF_SPECIAL_INSTRUCTION',

- 'SPECIAL_INSTRUCTIONS', 'SPECIAL_INSTRUCTION',

- 'SPECIAL_INSTRUCTIONS', 'SPECIAL_INSTRUCTION_IINE' /

C

C

Program variable names (value and null indicator) for screen fields.

data varblI/':fISTI:nISTI',':fICSN:nICSN' ' :fIOSIt:nIOS1',

. ':fISI1t:nISII',':siline:ifnsil'।

C

Possessor screen's field descriptors and field sizes.

character*80 fieldT (23), fidatT (23)

character*30 atrbt $(2,9)$

character $* 14$ varblT (9)

character*1 fitypT(23)

integer*4 fdlenT (23), fdrow (23), fdcolT (23), ficolT (23), mxnoct (23),

- farndT $(3,23)$, firndT $(3,23)$, !Field renditions (add/update/search).

- numfaT $/ 23 /$ !The number of fields.

integer*2 ifnulT(23), !NuIl indicator (-1 if null, 0 otherwise).

- ifrqdT (23) $/ 23 * 0 \%$ ! points to if-required dependent field.

- iditoT(23) $/ 23 * 0 /$ ! Points to allowed field for dittoing.

logical ifmodT(23) ! Has a field been modified in an Update? common/dbdatT/ fieldT, fidatT, fdlenT, fdrowT, fdcolT, ficolT, mxnocT,

- fdrndT, firndT, numfdT, fitypT, ifnulT, atrbt $T$, varblT, ifrqdT, ifmodT,

- iditoT

$\mathrm{C}$
$\mathrm{C}$
$\mathrm{C}$

Length-specified character fields for equivalences ( $f$ for field).

character fTSTI*12, fTCSN*60, fTPSN1 *3, fTPN1*25, fTPPN1*12, fTPA $1 * 60$,

. ITPPR $1 * 50$, ITDAB1 16, ITDRB1 16, , ITPSN2 $* 3$, ITPN2 $* 25$, fTPPN2 $* 12$, fTPA2 *60,

- ITPPR 2 *50, ITDAB2 *6, fTDRB2 *6, fTPSN $3 * 3$, fTPN3 325, ITPPN3 312, ITPA $3 * 60$,

- $\{T P P R 3 * 50$, ITDAB $3 * 6$, ITDRB $3 * 6$,

- fTPN1t*25, fTPPN1t*12, fTPA1t*60, fTPPR1t*50 !Temporary fields.

$\mathrm{C}$
$\mathrm{C}$
$\mathrm{C}$

Integer field storage (i for integer).

integer*4 iTPSN1, iTDAB1, iTDRB1 


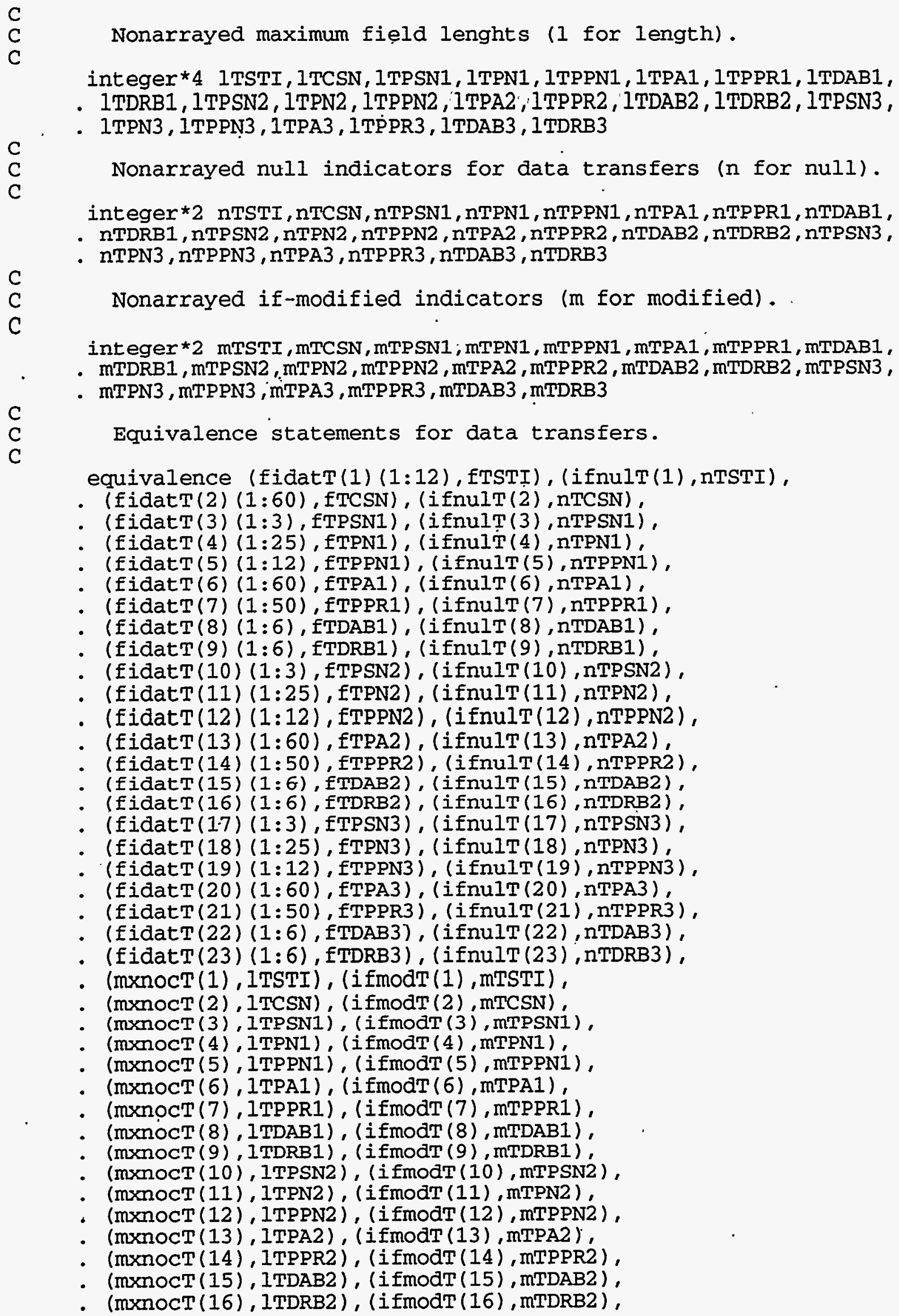


- (mxnoct (17), ITPSN3), (ifmodT (17), mTPSN3),.

- (mXnoct (18), 1TPN3), (ifmodT (18), mTPN3),

- (mxnoct (19), 1TPPN3), (i $\operatorname{mmodT}(19), \operatorname{mTPPN} 3)$,

- (mXnoct (20), ITPA3), (ifmodT (20), mTPA3),

. (mXnocT (21), ITPPR3), (ifmodT (21), mTPPR3),

- (mxnocT (22), ITDAB3), (i imodT (22) , mTDAB3),

- (mXnocT (23), ITDRB3), (ifmodT (23), mTDRB3)

C

Database table and attribute names for screen fields.

data atrbtT/

- 'TRACKING', 'SAMPLE_TRACKING_ID',

. 'SAMPLE_INFORMATION' ', CUSTOMER_SAMPLE_NAME',

- 'TRACKINNG', 'POSSESSOR_SEQUENCE_NUMBER',

- 'TRACKING', 'POSSESSOR_NAME',

- 'TRACKING', 'POSSESSOR_PHONE_NUMBER',

. 'TRACKING'.,' POSSESSOR_ADDRESS',

- 'TRACKING', 'POSSESSOR_POSSESSION_REASON' ,

- 'TRACKING', 'DATE_ACCEPTED_BY_POSSESSOR',

- 'TEACKING', 'DATE_RELINQUISHED_BY_POSSESSOR',

C

Program variable names (value and null indicator) for screen fields.

data varblT/':fTSTI:nTSTI',' : fTCSN:nTCSN',':iTPSN1:nTPSN1',

' ': fTPN1 : nTPN1',' : ITPPN1 : nTPPN1',' : ETPA1 :nTPA1', ' : fTPPR1 :nTPPR1',

- ' : iTDAB1 : ITDAB1',' : iTDRB1:nTDRB1'/

C

Possessor name list field descriptors and sizes.

character*80 fieldN $(4,15)$

integer 4 falenN $(4,15)$, fdrown (15), fdcolN(15), ficolN (15),

- numfdN !The number of fields.

common /1satatN/ fieldN, fdlenN, fdrown, fdcolN, ficoln, numfdN

$\mathrm{C}$

C For the Main screen, ...

C

C Set defaults for display field renditions now so they can be modified

C with each field as necessary. Set all input fields to blanks.

$\mathrm{C}$

do $i=1$, numfdM

fidatM $(i)=b l a n k s$

do $j=1,3$ ! 1 for adding, 2 for updating, and 3 for searching. fdrndM $(j, i)=$ foptnl enddo

C

enddo

fieldM(1)='Tracking ID:'

fdlenM $(1)=12$

fdrow $(1)=2$

$\operatorname{fdcolm}(1)=1$

ficolm(1) $=15$

$\operatorname{mxnocm}(1)=12$

C

fitypM $(1)=' I$ '.

fieldm(2) =' Date 'Entered:'

falenM $(2)=13$

fdrowm $(2)=2$

$\operatorname{facolm}(2)=31$

$\mathrm{fiCOlM}(2)=46$

$\operatorname{mxnocm}(2)=6$ 
C

$\operatorname{fitypM}(2)={ }^{\prime} d{ }^{\prime}$

do $i=1,2$

do $j=1,2$ ! Fixed when adding and updating. farndM $(j, i)=$ ffixed

, enddo

C enddo

fieldM( 3$)=$ ' Completed:'

fdlenM $(3)=10$

$\operatorname{fdrowM}(3)=2$

$\operatorname{fdcolM}(3)=56$

$\operatorname{ficolM}(3)=68$

$\operatorname{mXnocM}(3)=6$

C

fitypM $(3)=' d '$

fieldM $(4)=$ 'Customer' 's Sample ID:'

fdlenM $(4)=22$

fdrowM $(4)=3$

fdcolm $(4)=4$

ficolm $(4)=27$

$\operatorname{mxnocM}(4)=12$

C

$$
\text { fitypM }(4)=' a^{\prime}
$$

fieldM(5)='Desired Completion Date:'

falenM $(5)=24$

farowM $(5)=3$

fdcolm $(5)=45$

ficolm $(5)=71$

$\operatorname{mxnocM}(5)=6$

fitypM (5) = 'd'

do $j=1,2$ ! Required when adding and updating. fdrndM $(j, 5)=$ freqd

C enddo

$$
\begin{aligned}
& \text { fieldM }(6)=' \text { Project:' } \\
& \text { fdlenM }(6)=8 \\
& \text { fdrowM }(6)=4 \\
& \operatorname{fdcolM}(6)=4 \\
& \operatorname{ficol}(6)=14 \\
& \operatorname{mxnocM}(6)=40 \\
& \text { fitypM }(6)=' a '
\end{aligned}
$$

C

fieldM $(7)=$ 'Charge \#:'

fdlenM $(7)=9$

fdrowM $(7)=4$

fdcolM $(7)=58$

ficolm $(7)=69$

$\operatorname{mxnocM}(7)=9$

C

$$
\text { fitypM }(7)=' 1 \text { ' }
$$

$$
\begin{aligned}
& \text { fieldM }(8)=\text { 'Sample Name:' } \\
& \text { fdlenM }(8)=12 \\
& \text { fdrowM }(8)=5 \\
& \text { fdcolM }(8)=4 \\
& \text { ficolm }(8)=18 \\
& \operatorname{mxnocm}(8)=60 \\
& \text { fitypM }(8)=' a ' \\
& \text { iditom }(8)=4
\end{aligned}
$$

C 
fieldM(9)=' Sample Type:'

fdlenM $(9)=12$

fdrowM $(9)=6$

$\operatorname{fdColM}(9)=4$

ficolM $(9)=18$

$\operatorname{mxnocM}(9)=10$

C

fitypM $(9)=' a$ '

fieldM(10)='Sample Size:'

fdlen $(10)=12$

farowM $(10)=6$

facolm $(10)=32$

ficolM $(10)=46$

$\operatorname{mxnOcM}(10)=5$

C

$\operatorname{fitypM}(10)=' n$ '

fieldm(11)='Size Units:'

fdlenM $(11)=11$

$\operatorname{fdrowM}(11)=6$

$\operatorname{fdcolM}(11)=55$

ficolm $(11)=68$

$\operatorname{mxnOcM}(11)=5$

C

fitypM $(11)=' a$ '

fielaM(12) ='Collection Date:'

fdlenM $(12)=16$

fdrow $(12)=7$

$\operatorname{fdcolm}(12)=4$

ficolm $(12)=22$

$\operatorname{mxnocM}(12)=6$

fitypM $(12)=' d$ '

C

do $i=7,12$

do $j=1,2 \quad$ ! Required when adding and updating. fdrndm $(j, i)=$ freqd enddo

C enddo

fieldm(13)='Time:'

fdlenM $(13)=5$

fdrowM $(13)=7$

fdcolm $(13)=32$

fiColm $(13)=39$

$\operatorname{mxnocM}(13)=5$

fitypM $(13)=' t$ '

C

fieldM(14)='Special Instructions?'

fdlenM $(14)=21$

fdrowM $(14)=7$

fdcolm $(14)=48$

ficolm $(14)=71$

$\operatorname{mxnOcM}(14)=1$

C

fitypM $(14)=$ '?'

fieldM(15)='HP Surveyed?'

fdlenM $(15)=12$

fdrowM $(15)=8$

fdcolm $(15)=4$

$\operatorname{ficolM}(15)=18$

$\operatorname{mxnocM}(15)=1$ 
$\operatorname{fitypM}(15)={ }^{\prime} \cdot$
$\operatorname{ifrgdM}(15)=16$

fielaM(16)='If Yes; Sample Activity (mrem/h):'

falenM $(16)=33$

fdrowM $(16)=8$

$\operatorname{fdcolm}(16)=23$

ficolm $(16)=58$

$\operatorname{mxnocM}(16)=6$

fitypM $(16)=$ ' $n$ '

C

ifrqdM $(16)=15$

C

$$
\begin{aligned}
& \text { fieldM }(17)=\text { 'submitter:' } \\
& \text { fdlenM }(17)=10 \\
& \text { fdrowM }(17)=9 \\
& \text { fdcolm }(17)=1 \\
& \text { ficolM }(17)=21 \\
& \operatorname{mxnocM}(17)=25 \\
& \text { fitypM }(17)=' a '
\end{aligned}
$$

C

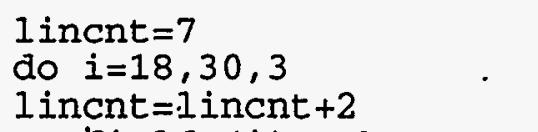

lincnt $=1$ incnt +2

fieldM(i)='Phone Number:'

fdlenM $(i)=13$

fdrowM $(i)=$ lincnt

fdcolm $(i)=50$

ficolm $(i)=65$

$\operatorname{mxnocm}(i)=12$

fitypM $(i)=$ ' $a$ '

ifrqdM $(i)=i-1$

if (i.ne. 18) iditom( $i)=i-3$

C

fieldM $(i+1)=$ 'Adaress:'

fdlenM $(i+1)=8$

fdrow $(i+1)=1$ incnt +1

$\operatorname{fdcolM}(i+1)=4$

ficolm $(i+1)=14$

$\operatorname{mxnocM}(i+1)=60$.

fitypM $(i+1)=' a$ '

ifrqdM $(i+1)=i-1$

if (i.ne.18) iditom $(i+1)=i-2$

enddo

do $i=17,19$

do $j=1,2$ ! Required when adding and updating. $\operatorname{fdrndM}(j, i)=$ freqd enddo

enddo

C

fieldM $(20)=$ 'Technical contact:'

falen $(20)=18$

fdrow $(20)=11$

$\operatorname{fdcol} M(20)=1$

ficolm $(20)=21$

$\operatorname{mXnOCM}(20)=25$

fitypM $(20)=' a$ '

C

iditoM $(20)=17$

fieldM(23) ='send Resuits To:' 
fdlenM $(23)=16$

farowM $(23)=13$

$\operatorname{fdColm}(23)=1$

$\operatorname{ficolm}(23)=21$

$\operatorname{mxnocM}(23)=25$

fitypM $(23)=$ ' $a$ '

$\operatorname{iditoM}(23)=20$

C

fieldM(26)='Sample Pickup By:'

fdlenM $(26)=17$

fdrowM $(26)=15$

$\mathrm{fdcolM}(26)=1$

ficolm $(26)=21$

$\operatorname{mxnocM}(26)=25$

fitypM $(26)={ }^{\prime} a$ '

iditom $(26)=23$

C

do $i=23,28$

do $j=1,2$ ! Required when adding and updating. $\operatorname{IdrndM}(j, i)=f r e q d$. enddo

C enddo

fieldM(29) ='Current Possessor:'

fdlenM $(29)=18$

fdrowM $(29)=17$

$\operatorname{fdcolM}(29)=1$

$f i \operatorname{col} M(29)=21$

$\operatorname{mxnocm}(29)=25$

fitypM $(29)=' a$ '

C

do $i=29,31$

fdrndM $(1, i)=$ ffixed $\quad$ Fixed when adding and updating. farndM $(2, i)=$ ffixed enddo

C

fieldM(32) ='Chain of Custody Number:'

fdlenM $(32)=24$

fdrowm $(32)=19$

fdcolm $(32)=1$

ficolm $(32)=27$

$\operatorname{mxnOcM}(32)=10$

C

$\operatorname{fitypM}(32)=' a$ '

fieldM(33)='Analyses Needed: Gross Alpha-Beta?'

falenM $(33)=35$

farowm (33) $=20$

$\operatorname{fdcolm}(33)=1$

ficolm $(33)=38$

$\operatorname{mxnocM}(33)=1$

C

fitypM $(33)=$ '?'

fieldM(34)=:'Alpha?'

falenM $(34)=6$

fdrow $(34)=20$

$\operatorname{facolm}(34)=43$

$\mathrm{ficolM}(34)=51$

$\operatorname{mxnocM}(34)=1$

C

fitypM $(34)=$ '?' 


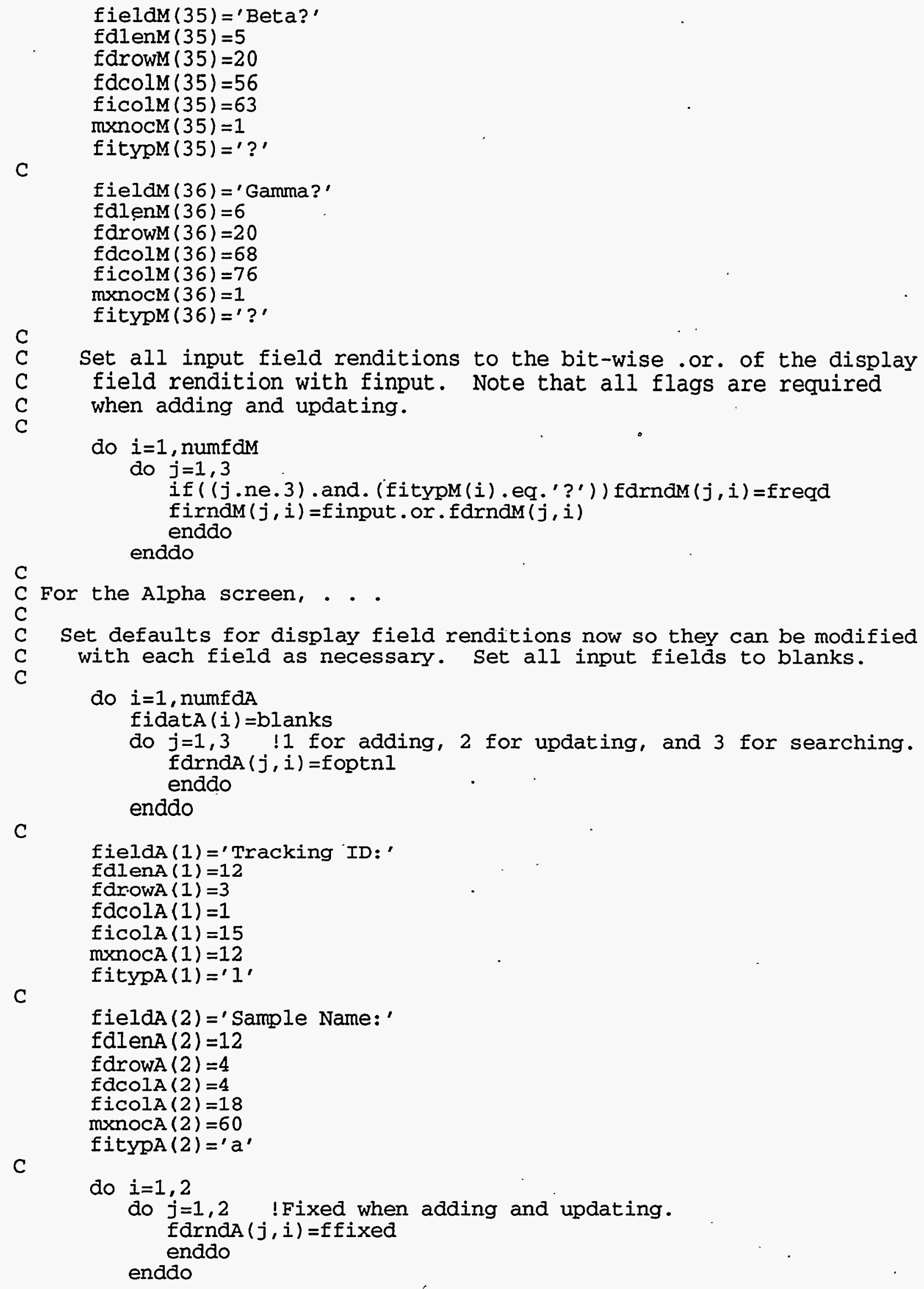


C

fieldA (3) =' Uraniur, sotopes?'

fdlenA $(3)=17$

fdrowa $(3)=6$

$\operatorname{fdcolA}(3)=1$

ficolA $(3)=20$

$\operatorname{mxnocA}(3)=1$

C

fitypa $(3)=$ '?'

lincnt $=4$

do $i=4,34,5$

lincnt $=1$ incnt +2

fieldA $(i)=$ 'Needed by:'

fdlenA $(i)=10$

fdrowA (i) $=1$ incnt

fdcolA $(i)=36$

$\mathrm{ficolA}(\mathrm{i})=48$

$\operatorname{mxnOCA}(i)=6$

fitypA $(i)=$ 'd'

ifrgdA $(i)=i-1$

C

if (i.ne.4) iditoA (i) $=i-5$

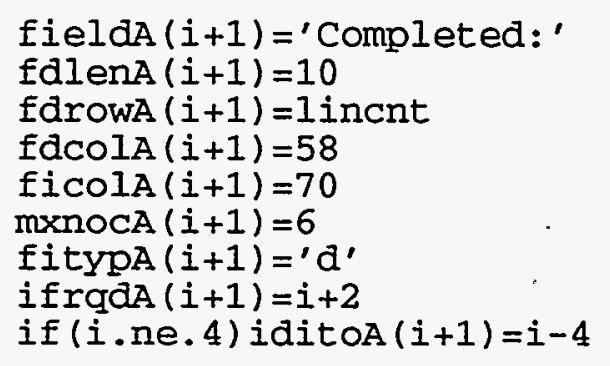

C

fieldA $(i+2)=$ 'Results report citation:'

fdlenA $(i+2)=24$

farowA $(i+2)=1$ incnt +1

$\operatorname{facolA}(i+2)=4$

ficolA $(i+2)=30$

$\operatorname{mxnocA}(i+2)=20$

fitypA $(i+2)=' a$ '

ifrgda $(i+2)=i+1$

if (i.ne.4) iditoA $(i+2)=i-3$.

C

fieldA $(i+3)=$ 'Special Instructions?'

falenA $(i+3)=21$

fdrowA $(i+3)=$ lincnt +1

fdcolA $(i+3)=54$

ficolA $(i+3)=77$

$\operatorname{mxnocA}(i+3)=1$

fitypA $(i+3)=$ '?'

ifrgdA $(i+3)=i-1$

if $(i . n e .4)$ iditoA $(i+3)=i-2$

C

enddo

fieldA (8) ='Thorium isotopes?'

fdlenA $(8)=17$

fdrowA $(8)=8$

fdcola $(8)=1$

ficolA $(8)=20$

$\operatorname{m\times nOcA}(8)=1$

fitypa (8) =' ?' 
C

fieldA (13)='Plutonium isotopes?'

fdlenA $(13)=19$

fdrowA $(13)=10$

fdcola $(13)=1$

$\operatorname{ficolA}(13)=22$

$\operatorname{mxnOCA}(13)=1$

C

fitypa (13) ='?'

C

fieldA (18) ='Am-241 separate from Pu-238?'

fdlenA $(18)=28$

farowa $(18)=12$

$\mathrm{fdcolA}(18)=1$

$\mathrm{ficolA}(18)=31$

$\operatorname{mxnocA}(18)=1$

fitypA $(18)=$ ' ?'

fieldA (23) ='Am-241 combined with Pu-238?'

fdlenA $(23)=28$

fdrowA $(23)=14$

fdcolA $(23)=1$

ficolA $(23)=31$

$\operatorname{mxnOcA}(23)=1$

C

fitypA $(23)=$ '?'

C

fieldA (28)='Total Spectrometric Alpha?'

fdlenA $(28)=26$

fdrowA $(28)=16$

$\operatorname{fdcolA}(28)=1$

ficolA $(28)=29$

$\operatorname{mXnOCA}(28)=1$

fitypA (28) ='?'

fieldA $(33)=$ 'Other?'

fdlenA $(33)=6$

fdrowa $(33)=18$

fdcolA $(33)=1$

fiColA $(33)=9$

$\operatorname{mxnocA}(33)=1$

fitypa (33) ='?'

$C$
$C$
$C$
$C$
$C$

Set all input field renditions to the bit-wise or. of the display field rendition with finput. Note that some flags are required when adding and updating.

do $i=1$, numfdA do $j=1,3$

if ( (j.ne.3) . and. (fitypA (i) .eq.'?') . and.

(fieldA (i).ne.'Special Instructions?')) fdrndA $(j, i)=$ freqd

firndA $(j, i)=f i n p u t$. or. fdrndA $(j, i)$

enddo

enddo

C For the Beta screen, ..

C

C Set defaults for display field renditions now so they can be modified

C with each field as necessary. Set all input fields to blanks.

C

do $i=1$, numfdB

fidat $B(i)=$ blanks 
C

do $j=1,3$ ! for adding, 2 for updating, and 3 for searching. fdrndB $(j, i)=$ foptn 1

enddo . enddo

fieldB $(1)=$ 'Tracking ID:'

fdlen $B(1)=12$

fdrowB $(1)=2$

$\operatorname{fdcolB}(1)=1$

$\mathrm{ficolB}(1)=15$

$\operatorname{mxnocB}(1)=12$

C

fitypB $(1)=' 1$ '

fieldB(2)=' Sample Name:'

fdlenB $(2)=12$

farowB $(2)=3$

$\mathrm{fdcolB}(2)^{\circ}=4$

$\mathrm{ficolB}(2)=18$

$\operatorname{mxnocB}(2)=60$

fityp $B(2)=' a$ '

do $i=1,2$

do $j=1,2 \quad$ ! Fixed when adding and updating. fdrndB $(j, i)=$ ffixed enddo

C

enddo

fieldB (3) =' Strontium-90?'

fdlenB $(3)=13$

farowB $(3)=5$

$\operatorname{fdcolB}(3)=1$

$\mathrm{ficolB}(3)=16$

$\operatorname{mxnOcB}(3)=1$

C

fitypB $(3)=$ '?'

lincnt $=2$

do $i=4,28,8$

lincnt $=1$ incnt +3

fieldB(i)='Length of count:'

fdlenB $(i)=16$

fdrowB $(i)=1$ incnt

$\operatorname{fdcolB}(i)=29$

$f i \operatorname{colB}(i)=47$

$\operatorname{mxnOcB}(i)=4$

fitypB $(i)=$ ' $n$ '

ifrgdB $(i)=i+1$

if (i.ne. 4) iditoB $(i)=i-8$

C

fielab $(i+1)=$ 'min or $h r$ ?'

fdlen $B(i+1)=10$

fdrowB $(i+1)=1$ incnt

$f d \operatorname{colB}(i+1)=55$

$f i \operatorname{colB}(i+1)=67$

$\operatorname{mxnOCB}(i+1)=1$

fitypB $(i+1)=' u$ '

ifrgdB $(i+1)=i$

C

if (i.ne. 4$)$ idito $(i+1)=i-7$

fieldB $(i+2)=$ 'Results needed by: date: ' fdlenB $(i+2)=25$ 
fdrowB $(i+2)=$ lincnt +1

$f d \operatorname{col} B(i+2)=4$

$f i \operatorname{colB}(i+2)=31$

$\operatorname{mxnocB}(i+2)=6$

fitypB $(i+2)=' d$ '

ifrqd $B(i+2)=i-1$

if $(i . n e .4)$ iditoB $(i+2)=i-6$

C

fieldB $(i+3)=$ 'time:'

falenB $(i+3)=5$

fdrowB $(i+3)=1$ incnt +1

fdColB $(i+3)=41$

ficolB $(i+3)=48$

$\operatorname{mxnOCB}(i+3)=5$

fitypB $(i+3)=$ ' $t$ '

C

if $(i$.ne. 4$)$ iditoB $(i+3)=i-5$

fieldB $(i+4)=$ ' Completed:'

fdlen $B(i+4)=10$

fdrowB $(i+4)=$ inent +1

$f d \operatorname{colB}(i+4)=57$

$\mathrm{ficolB}(i+4)=69$

$\operatorname{mxnOCB}(i+4)=6$

fitypB $(i+4)=$ ' $d$ '

ifrgdB $(i+4)=i+5$

C

if $(i . n e .4)$ iditoB $(i+4)=i-4$

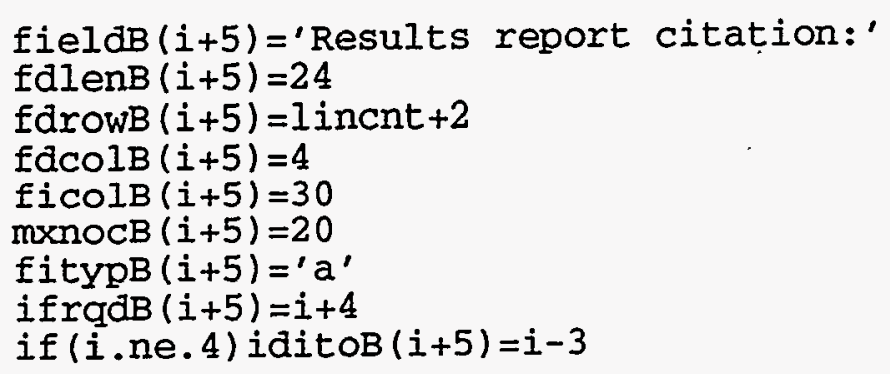

C

fieldB $(i+6)=$ 'Special Instructions?'

fdlenB $(i+\sigma)=21$

fdrowB $(i+6)=1$ incnt +2

fdcolB $(i+6)=54$

ficolB $(i+6)=77$

$\operatorname{mxnOCB}(i+6)=1$

fitypB $(i+6)=$ '?'

ifradB $(i+6)=i-1$

if $(i$.ne. 4$)$ iditoB $(i+6)=i-2$

enddo

C

fieldB $(11)=$ 'Strontium -89 and -90 ?'

fdlen $(11)=21$

fdrowB $(11)=8$

$\operatorname{IdCOlB}(11)=1$

ficolB $(11)=24$

$\operatorname{mxnocB}(11)=1$

C

fitypB $(11)=$ '?'

fieldB $(19)=$ 'Total strontium?'

fdlenB $(19)=16$

farowB $(19)=11$

$\mathrm{fdColB}(19)=1$ 
$f i \operatorname{colB}(19)=19$

$\operatorname{mxnocB}(19)=1$

C

fitypB (19) ='?'

fieldB (27) ='Tritium?'

fdlen $B(27)=8$

farowB $(27)=14$

$\mathrm{fdcolB}(27)=1$

ficolB $(27)=11$

$\operatorname{mxnocB}(27)=1$

C

fitypB $(27)=$ '?'

fieldB (35) ='Other?'

fdlen $B(35)=6$

farowB $(35)=17$

$\operatorname{fdcolB}(35)=1$

$\mathrm{ficolB}(35)=9$

$\operatorname{mxnocB}(35)=1$

C

fitypB (35) ='?'

fieldB $(36)=$ 'Length of count:'

fdlenB $(36)=16$

fdrowB $(36)=17$

fdcolB $(36)=29$

ficolB $(36)=47$

$\operatorname{mxnocB}(36)=4$

fitypB $(36)=' n$ '

C

iditoB $(36)=28$

C

$$
\begin{aligned}
& \text { fieldB }(37)=' \text { min or hr?' } \\
& \text { fdienB }(37)=10 \\
& \text { fdrowB }(37)=17 \\
& \text { fdcolB }(37)=55 \\
& \text { ficolB }(37)=67 \\
& \text { mxnocB }(37)=1 \\
& \text { fitypB }(37)=' u^{\prime} \\
& \text { ifrqdB }(37)=36 \\
& \text { iditoB }(37)=29
\end{aligned}
$$

fieldB (38) ='Nickel-63?'

fdlen $B(38)=10$

fdrowB $(38)=18$

$\mathrm{fdcolB}(38)=4$

$\mathrm{ficolB}(38)=16$

$\operatorname{mxnOCB}(38)=1$

fitypB (38) =' ?'

ifrqdB $(38)=35$

C

fieldB (39) ='Iron-55?'

fdlenB $(39)=8$

fdrowB $(39)=18$

fdcolB $(39)=21$

ficolB $(39)=31$

$\operatorname{mxnocB}(39)=1$

fitypB $(39)=$ '?'

C

ifrqdB $(39)=35$

fieldB $(40)=$ 'Sulfur-35?'

fdlen $(40)=10$

fdrow $(40)=18$ 


$$
\begin{aligned}
& \text { fdcolB }(40)=36 \\
& \operatorname{ficolB}(40)=48 \\
& \operatorname{mxnocB}(40)=1 \\
& \text { fitypB }(40)={ }^{\prime} ? ' \\
& \text { ifrgdB }(40)=35
\end{aligned}
$$

C

C

fieldB $(41)=$ 'Plutonium-241?'

fdlenB $(41)=14$

fdrowB $(41)=18$

fdcolB $(41)=53$

$\mathrm{ficolB}(41)=69$

$\operatorname{mxnOcB}(41)=1$

fitypB $(41)=$ '?'

ifrqaB $(41)=35$

fieldB (42)='Results needed by: date:'

fdlenB $(42)=25$

fdrowB $(42)=19$

fdCoIB $(42)=4$

ficolB $(42)=31$;

$\operatorname{mxnocB}(42)=6$

fitypB $(42)=$ 'd'

ifrgdB $(42)=35$

iditoB $(42)=30$

C

fieldB $(43)=$ 'time: '

fdlenB $(43)=5$

fdrowB $(43)=19$

fdcolB $(43)=41$

ficolB $(43)=48$

$\operatorname{mxnOCB}(43)=5$

fitypB $(43)=$ ' $t$ '

C

iditoB $(43)=31$

fieldB (44) =' Completed: '

fdlenB $(44)=10$

fdrowB $(44)=19$

fdcolB $(44)=57$

ficolB $(44)=69$

$\operatorname{mxnocB}(44)=6$

fitypB $(44)=' d$ '

ifrqdB $(44)=45$

C

iditoB $(44)=32$

fieldB (45) ='Results report citation:'

fdlenB $(45)=24$

fdrowB $(45)=20$

fdcolB $(45)=4$

ficolB $(45)=30$

$\operatorname{mxnOCB}(45)=20$

fitypB $(45)=$ ' $a$ '

ifrgab $(45)=44$

iditoB $(45)=33$

C

fieldB $(46)=$ 'Special Instructions?'

fdlenB $(46)=21$

fdrowB $(46)=20$

fdcolB $(46)=54$

$\mathrm{ficolB}(46)=77$

$\operatorname{mxnocB}(46)=1$ 


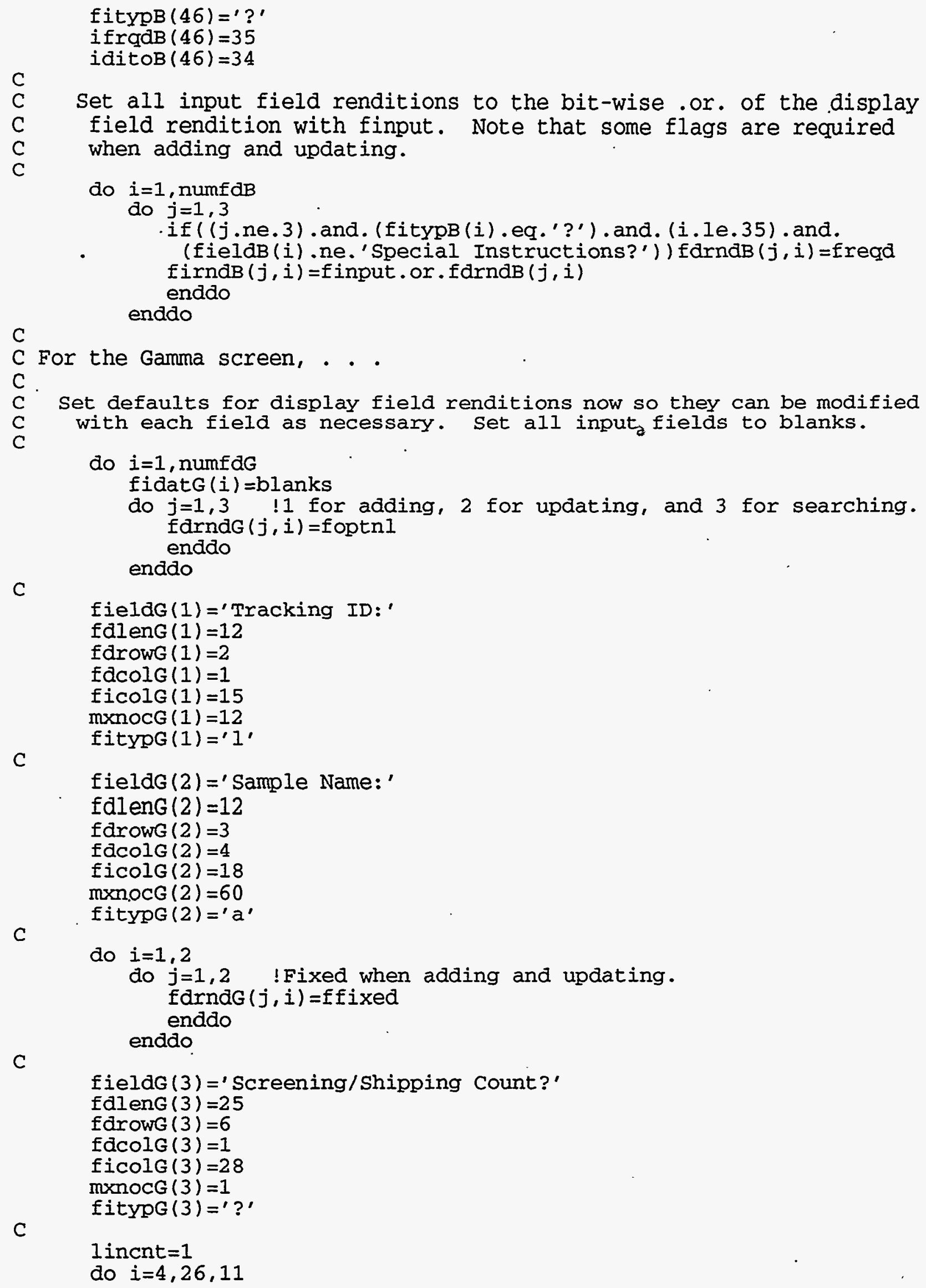


lincnt $=1$ incnt +5

fieldG(i)='Length of count:'

fdleng $(i)=16$

fdrowg $(i)=1$ incnt

fdcolg $(i)=37$

ficolg $(i)=55$

$\operatorname{mxnOCG}(i)=4$

fitypG $(i)=$ ' $n$ '

C

if (i.ne.4) iditog (i) $=i-11$

fieldG $(i+1)=' \min$ or $\mathrm{hr}$ ?'

fdleng $(i+1)=10$

farowg $(i+1)=1$ incnt

fdcolg $(i+1)=63$

ficolg $(i+1)=75$

$\operatorname{mxnOCG}(i+1)=1$

fitypg $(i+1)=$ 'u'

ifrgdG $(i+1)=i$

C

if $(i$.ne. 4$)$ iditog $(i+1)=i-10$

fieldG $(i+2)=$ 'Results needed by: date:'

fdleng $(i+2)=25$

fdrowG $(i+2)=$ lincnt +1

facolg $(i+2)=4$

ficolg $(i+2)=31$

$\operatorname{m\times nOCG}(i+2)=6$

fitypg $(i+2)=' d$ '.

ifrgdG $(i+2)=i-1$

if $(i$.ne.4) iditog $(i+2)=i-9$

C

fieldG $(i+3)=$ 'time:' .

fdleng $(i+3)=5$

fdrowg $(i+3)=1$ incnt +1

fdcolg $(i+3)=41$

ficolg $(i+3)=48$

mXnocG $(i+3)=5$

fitypg $(i+3)=$ ' $t$ '

C

if (i.ne.4) iditog $(i+3)=i-8$

fieldG $(i+4)=$ 'Completed:'

fdleng $(i+4)=10$

fdrowg $(i+4)=1$ incnt +1

fdcolg $(i+4)=57$

ficolG $(i+4)=69$

$\operatorname{m\times nOCG}(i+4)=6$

fitypg $(i+4)=$ ' $d$ '

ifrgdG $(i+4)=i+8$

if $(i$.ne.4) iditog $(i+4)=i-7$

C

fieldG $(i+5)=$ 'Date counted:'

fdleng $(i+5)=13$

farowg $(i+5)=1$ incnt +2

fdcolg $(i+5)=4$

ficolg $(i+5)=19$

$\operatorname{mxnocG}(i+5)=6$

fitypg $(i+5)={ }^{\prime} d$ '

C

ifrqdG $(i+5)=i+4$

fieldG $(i+\sigma)=$ RML Spectral ID:'

fdleng $(i+6)=16$ 
C

C

C

C

C

fdrowG $(i+6)=1$ incnt +2

fdcolg $(i+6)=29$

ficolg $(i+6)=47$

$\operatorname{mxnocG}(i+6)=14$

fitypg $(i+6)=' 1$ '

ifrgdG $(i+6)=i+5$

fieldG $(i+7)=$ 'Recount?'

fdleng $(i+7)=8$

fdrowg $(i+7)=$ lincnt +2

fdcolg $(i+7)=65$

ficolg $(i+7)=75$

mxnocG $(i+7)=1$

fitypG $(i+7)=$ '?'

ifrgdG $(i+7)=i+5$

fieldG $(i+8)=$ 'Results report citation:'

fdleng $(i+8)=24$

fdrow $(i+8)=1$ incnt +3

fdcolg $(i+8)=4$

ficolg $(i+8)=30$

MXnocG $(i+8)=20$.

fitypg $(i+8)=' a$ '

ifrgdG $(i+8)=i+4$

if $(i . n e .4)$ iditog $(i+8)=i-3$

fieldG $(i+9)=$ 'Special Instructions?'

fdleng $(i+9)=21$

fdrowg $(i+9)=1$ incnt +3

fdcolg $(i+9)=54$

ficolg $(i+9)=77$

$\operatorname{mXnOCG}(i+9)=1$

fitypG $(i+9)='$ ?'

ifradG $(i+9)=i-1$

if (i.ne.4) iditog $(i+9)=i-2$

enddo

fieldG(14)='Full Isotopic Gamma Analysis?'

fdleng $(14)=29$

fdrowG $(14)=11$

fdcolg $(14)=1$

fico' $1 \mathrm{G}(14)=32$

$\operatorname{mXnOCG}(14)=1$

fitypg (14) ='?'

fieldG(25) ='Other Gamma Analysis?'

fdleng $(25)=21$

fdrowG $(25)=16$

fdcolg $(25)=1$

ficolg $(25)=24$

$\operatorname{mXnOCG}(25)=1$

fitypg $(25)=$ '?'

C

C

$\mathrm{C}$

C

Set all input field renditions to the bit-wise .or. of the display field rendition with finput. Note that some flags are required when adding and updating.

do $i=1$, numfdG

do $j=1,3$

if ( (j.ne.3) .and. (fitypg (i).eq.'?') . and. 
- (fieldG(i).ne. 'Recount?') . and.

- (fieldG(i).ne.'Special Instructions?')) fdrndG $(j, i)=$ fregd firndG $(j, i)=f i n p u t$.or. fdrndG $(j, i)$

enddo enddo

C

C For the Gross Alpha-Beta screen, . .

C

C Set defaults for display field renditions now so they can be modified

$\dot{\mathrm{C}}$ with each field as necessary. Set all input fields to blanks.

do $i=1$, numfdC

fidatc $(i)=$ blanks

do $j=1,3$ ! for adding, 2 for updating, and 3 for searching. fdrndc $(j, i)=$ foptn 1

enddo

C

fieldC (1)='Tracking ID: '

fdlenc $(I)=12$

farowC $(1)=5$

fdcolc $(1)=1$

ficolc (1) $=15$

$\operatorname{mxnocC}(1)=12$

C

fitypc $(1)=' 1$ '

fieldC (2) ='Sample Name:'

fdlenc $(2)=12$

fdrowC $(2)=6$

$\mathrm{fdcolc}(2)=4$

$\mathrm{ficolc}(2)=18$

$\operatorname{mxnocC}(2)=60$

C

fitypc $(2)=' a$ '

do $i=1,2$

do $j=1,2 \quad$ ! Fixed when adding and updating. fdrndC $(j, i)=f$ fixed endao

enddo

C

fieldC $(3)=$ 'Gross Alpha-Beta for Air Filters?'

fdlenc $(3)=33$

farowC $(3)=8$

fdcolc $(3)=1$

ficolc $(3)=36$

$\operatorname{mxnocC}(3)=1$

C

fitypC $(3)=$ '?'

lincnt $=4$

do $i=4,12,8$

lincnt $=1$ incnt +5

fieldC $(i)=$ 'Length of count:'

falenc $(i)=16$

fdrowc $(i)=$ lincnt

fdcolc $(i)=4$

ficolc $(i)=22$

$\operatorname{mxnocC}(i)=4$

fitypC $(i)=$ 'n'

C

if (i.ne.4) iditoc (i) $=i-8$ 
fieldc $(i+1)=$ 'min or hr?'

fdlenc $(i+1)=10$

fdrowc $(i+1)=1$ incnt

facolc $(i+1)=30$

ficolc $(i+1)=42$

$\operatorname{mxnocC}(i+1)=1$

fitypc $(i+1)=' u$ '

ifrgad $(i+1)=i$

C

if (i.ne.4) iditoc $(i+1)=i-7$

fieldC $(i+2)=$ 'Results needed by: date:'

fdlenc $(i+2)=25$

fdrowC $(i+2)=$ incnt +1

fdcolc $(i+2)=4$

ficolc $(i+2)=31$

$\operatorname{mxnocC}(i+2)=6$

fitypC $(i+2)=$ ' $d$ '

ifrgdc $(i+2)=i-1$

if $(i$.ne. 4$)$ iditoc $(i+2)=i-6$

C

fieldc $(i+3)=$ 'time: '

falenc $(i+3)=5$

fdrowC $(i+3)=$ lincnt +1

facolc $(i+3)=41$

ficolc $(i+3)=48$

$\operatorname{mxnocC}(i+3)=5$

fitypc $(i+3)=' t$ '

C

if $(i$.ne. 4$)$ iditoc $(i+3)=i-5$

fieldC $(i+4)=$ ' Completed:'

falenc $(i+4)=10$

farowC $(i+4)=$ lincnt +1

facolc $(i+4)=57$

ficolc $(i+4)=69$

$\operatorname{mxnocC}(i+4)=6$

fitypc $(i+4)=$ ' $d$ '

ifradC $(i+4)=i+5$

if $(i . n e .4)$ iditoc $(i+4)=i-4$

C

fieldC $(i+5)=$ 'Results report citation:'

falenc $(i+5)=24$

farowC $(i+5)=1$ incnt +2

fdcolc $(i+5)=4$

ficolc $(i+5)=30$

$\operatorname{mxnocC}(i+5)=20$

fitypc $(i+5)={ }^{\prime} a^{\prime}$

ifrgac $(i+5)=i+4$

if $(i$.ne. 4$)$ iditoc $(i+5)=i-3$

C

fieldC $(i+6)=$ 'Special Instructions?'

fdlenc $(i+6)=21$

fdrowC $(i+6)=1$ incnt +2

fdcolc $(i+6)=54$

ficolc $(i+6)=77$

$\operatorname{mxnocC}(i+6)=1$

fitypc $(i+6)=$ '?'

ifrgdC $(i+6)=i-1$

if (i.ne.4) iditoc $(i+6)=i-2$

C

enddo 
fieldC $(11)=$ 'Gross Alpha-Beta for Other Samples?'

fdlenc $(11)=35$

fdrowC $(11)=13$

fdcolc $(11)=1$

ficolc $(11)=38$

$\operatorname{mxnocC}(11)=1$

fitypc $(11)=' ? \cdot$

C

C

Set all input field renditions to the bit-wise .or. of the display field rendition with finput. Note that some flags are required when adding and updating.

C

do $i=1$, numfdC

do $j=1,3$ if ( (j.ne.3) .and. (fitypc (i).eq.'?') . and.

- (fieldc $(i)$.ne.'Special Instructions?') ) farndc $(j, i)=$ freqd firndC $(j, i)=$ finput.or. $\operatorname{farndC}(j, i)$

enddo endalo

C

C For the Special Instruction screen, . .

C

Set defaults for display field renditions now so they can be modified with each field as necessary. Set all. input fields to blanks.

C

do $i=1$, numfdI

fidat $I(i)=$ blanks

do $j=1,3$ ! 1 for adding, 2 for updating, and 3 for searching. fdrndI $(j, i)=$ foptnl enddo

C enddo

fieldI (1) ='Tracking ID:'

fdlenI $(1)=12$

farowI $(1)=2$

$\operatorname{fdcol} I(1)=1$

ficolI $(1)=15$

$\operatorname{mxnocI}(1)=12$

$\operatorname{fitypI}(1)=' 1$ '

C

fieldI (2) =' Sample Name:'

fdlenI (2) $=12$

fdrowI $(2)=3$

fdcolI $(2)=4$

ficolI $(2)=18$

$\operatorname{mxnocI}(2)=60$

C

fitypI $(2)=' a$ '

do $i=1,2$

do $j=1,2$ ! Fixed when adding and updating. fdrndI $(j, i)=f f i x e d$ enddo

C enddo

do $i=3,31,2$

fieldI (i) ='From'

fdlenI $(i)=4$

fdrowI $(i)=3+(i+1) / 2$

fdcolI $(i)=4$

$f i \operatorname{col} I(i)=9$ 
$\operatorname{mxnocI}(i)=16$

fitypI (i) $=$ 'a'

fdrndI $(1, i)=$ ffixed !Fixed when adding.

C

fdrndI $(2, i)=$ ffixed !Fixed when updating.

C

C

C

C

C

C For the Possessor screen, . .

fieldI $(i+1)=':^{\prime}$

falenI $(i+1)=1$

farowI $(i+1)=3+(i+1) / 2$

fdcolI $(i+1)=25$

ficolI $(i+1)=28$

$\operatorname{mxnocI}(i+1)=50$

fitypI $(i+1)=$ ' $a$ '

endao

Set all input field renditions to the bit-wise .or. of the display field rendition with finput.

do $i=1$, numfaI

do $j=1,3$

firndI $(j, i)=f i n p u t . o r . f d r n d I(j, i)$

enddo enddo

C

C

C

C

Set defaults for display field renditions now so they can be modified with each field as necessary. Set all input fields to blanks.

do $i=1$, numfdT

fidat $(i)=b l a n k s$

do $j=1,3$ ! I for adding, 2 for updating, and 3 for searching. farnat $(j, i)=$ foptnl enddo

enddo

fieldT(1)='Tracking ID:'

fdlenT $(1)=12$

fdrowT $(1)=3$

$\mathrm{fdcolT}(1)=1$

ficolT $(1)=15$

$\operatorname{mxnocT}(1)=12$

C

fityp $T(1)=' 1$ '

fieldT(2)='Sample Name:'

fdlent $(2)=12$

fdrowT $(2)=4$

fdcolT $(2)=4$

$\mathrm{ficolT}(2)=18$

$\operatorname{mxnocT}(2)=60$

C

fitypT $(2)=' a$ '

do $i=1,2$

do $j=1,2$ ! Fixed when adding and updating. farnat $(j, i)=$ ffixed

enddo

C

lincnt $=1$

do $i=3,17,7$

lincnt $=1$ incnt +5 
fieldT $(i)=$ 'Possessor \#'

fdlenT $(i)=11$

fdrow $(i)=$ incnt

$\operatorname{fdcolT}(i)=1$

$\mathrm{ficolT}(i)=13$

$\operatorname{mxnocT}(i)=3$

fitypT $(i)=$ 'n'

fdrndT $(1, i)=$ ffixed !Fixed when adding and

C fdrndT $(2, i)=$ ffixed $\quad$ updating.

C

fieldT $(i+1)=$ ' $^{\prime}$

fdlenT $(i+1)=1$

farow $(i+1)=1$ incnt

$\operatorname{fdcolT}(i+1)=16$

ficolT $(i+1)=22$

$\operatorname{mxnocT}(i+1)=25$

fitypT $(i+1)=$ ' $a$ '

if (i.eq. 3 ) then

fdrndT $(1,(i+1))=$ ffixed

farndT $(2,(i+1))=$ ffixed else endif

ifrqdT $(i+1)=i-1$

fieldT $(i+2)=$ 'Phone Number:'

fdlenT $(i+2)=13$

farowt $(i+2)=1$ incnt

fdcolt $(i+2)=51$

ficolT $(i+2)=66$

$\operatorname{mxnocT}(i+2)=12$

fitypT $(i+2)=' a$ '

if (i.eq. 3) then

farndT $(1,(i+2))=$ ffixed farndT $(2,(i+2))=$ ffixed

! Fixed when adding and ! updating. else endif ifrqdT $(i+2)=i+1$

C

fieldT $(i+3)=$ 'Address:'

fdlenT $(i+3)=8$

fdrow $(i+3)=$ lincnt +1

fdcolT $(i+3)=4$

ficolT $(i+3)=14$

$\operatorname{mxnocT}(i+3)=60$

fitypT $(i+3)=' a$ '

if (i.eq. 3 ) then

farndT $(1,(i+3))=$ ffixed fdrndT $(2,(i+3))=$ ffixed

!Fixed when adding and ! updating. else ifrqdT $(i+3)=i+1$ endif

$C^{\prime}$

fieldT $(i+4)=$ 'Reason for possession:'

fdlenT $(i+4)=22$

fdrow $(i+4)=$ lincnt +2

$\operatorname{fdcolT}(i+4)=4$

ficolT $(i+4)=28$

$\operatorname{mxnocT}(i+4)=50$

fitypT $(i+4)=$ ' $a$ '

if (i.eq.3) then

! Fixed when adding and ! updating. 
C

C

C

C

C else

farndT $(1,(i+4))=$ ffixed $!$ Fixed when adding and endif

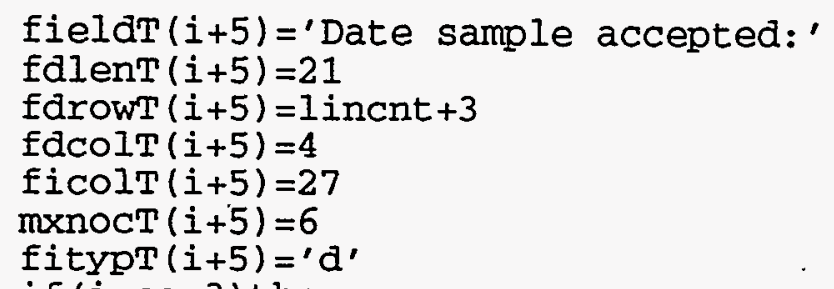

Set all input field renditions to the bit-wise .or. of the display field rendition with finput.

do $i=1$, numfdT

do $j=1,3$

firndT $(j, i)=f i n p u t$. or $f \operatorname{fdrndT}(j, i)$ enddo

enddo

Possessor name list data (set up to ease adding and removing names - note that the current limit is fifteen names).

numfdN $=1$

fieldN $(1$, numfdN) $=$ 'J. L. DOHERTY (JODIE)'

fdlenN $(1$, numfaN $)=21$

fieldN $(2$, numfdN $)=' 6-6573$ '

fdlenN $(2, \operatorname{numfdN})=6$

fieldN (3, numfdN)='RML, MS 7111; TRA-604, ROOM 123'

fdlenN $(3$, numfdN $)=31$

fieldN $(4$, numfdN $)=$ ' GAMMA SPECTRUM ACQUISITION'

fdlenN $(4$, numfdN $)=26$

numfdN=numfdN +1

fieldN $(1$, numfdN $)=$ 'T. J. HANEY (TOM)'

falenN $(1$, numfdN $)=17$

fieldN $(2$, numfdN $)=' 6-4158^{\prime}$

fdlenN $(2$, numfdN $)=6$

fieldN (3, numfdN) ='RMI, MS 7111; TRA-604, ROOM 126'

falenN $(3$, numfdN $)=31$

fieldN $(4$, numfdN $)=$ ' SAMPLE ROUTING COORDINATION' 
C

falenN $(4, \operatorname{numfdN})=27$

numfdN=numfdN +1

fieldN $(1$, numfdN $)=' R$. K. MURRAY (RON)'

fdlenN $(1$, numfaN $)=18$

fieldN $(2$, numfdN $)=' 6-4182$ '

fdlenN $(2, \operatorname{numfdN})=6$

fieldN (3, numfdN)='RML, MS 7111; TRA-604, ROOM 123'

fdlenN $(3$, numfdN $)=31$

fieldN (4, numfdN) ='GAMMA SPECTRUM ACQUISITION'

fdlenN $(4$, numfdN $)=26$

C

numfdN $=$ numfdN +1

fieldN $(1$, numfdN $)={ }^{\prime} C$. L. ROWSELL (CAL)'

fdlenN $(1$, numfaN $)=19$

fieldN $(2$, numfdN $)=' 6-4182^{\prime}$

fdlenN $(2$, numfdN $)=6$

fieldN (3, numfdN) ='RML, MS 7111; TRA-604, ROOM 123'

fdlenN $(3$, numfdN $)=31$

fieldN $(4$, numfdN $)=$ 'GROSS ALPHA-BETA COUNTING'

C

fdlenN $(4$, numfdN $)=25$

numfdN=numfaN+1

fieldN $(1$, numfdN $)=' C$. W. SILL (CLAUDE)'

fdlenN $(1$, numfdN $)=19$

fieldN $(2$, numfdN $)=' 6-0605$ '

fdlenN $(2$, numfdN $)=6$

fieldN (3, numfdN) ='RADIOCHEMISTRY, MS 7111; TRA-604, ROOM 111'

fdlenN $(3$, numfdN $)=42$

fieldN $(4$, numfdN $)=$ 'ALPHA ANALYSES'

fdlenN $(4$, numfdN $)=14$

C

numfdN $=$ numfdN +1

fieldN $(1$, numfdN $)=' D$. S. SILL (DAVE)'

fdlenN $(1$, numfdN $)=17$

fieldN $(2$, numfdN $)=' 6-8031$ '

fdlenN $(2$, numfdN $)=6$

fieldN (3, numfdN) ='RADIOCHEMISTRY, MS 7111; TRA-604, ROOM 110'

fdlenN $(3$, numfdN $)=42$

fieldN $(4$, numfdN $)=$ 'ALPHA ANALYSES'

fdlenN $(4$, numfdN $)=14$

C

numfdN $=$ numfdN +1

fieldN $(1$, numfaN) $=$ 'I. D. SMITH (LARRY)'

fdlenN $(1$, numfdN $)=19$

fieldN $(2, \operatorname{numfaN})=' 6-4405^{\prime}$

fdlenN $(2$, numfdN $)=6$

fieldN (3, numfdN)='RML, MS 7111; TRA-604, ROOM 123'

fdlenN $(3$, numfdN $)=31$

fieldN $(4$, numfdN $)=$ 'GAMMA SPECTRUM ACQUISITION'

C

fdlenN $(4$, numfdN $)=26$

numfdN=numfdN +1

fieldN $(1$, numfdN) $=' G$. K. TAYLOR (GENE)'

fdlenN $(1$, numfdN $)=19$

fieldN $(2$, numfdN $)=' 6-4041^{\prime}$

fdlenN $(2$, numfdN $)=6$

fielaN (3, numfaN)='RML, MS 7111; TRA-604, ROOM 123'

fdlenN $(3$, numfdN $)=31$

fieldN (4, numfdN) ='GAMMA SPECTRUM ACQUISITION' 
C

falenN $(4$, numfdN $)=26$

numfaN $=$ numfdN +1

fieldN $(1, \operatorname{numfdN})=$ 'L. A. WEINRICH (LOU)'

fdlenN $(1$, numfdN $)=20$

fieldN $(2$, numfdN $)=' 6-4404$ '

falenN $(2$, numfdN $)=6$

fieldN (3, numfdN) ='RADIOCHEMISTRY, MS 7111; TRA-661, ROOM 129/130'

fdlenN $(3$, numfdN $)=46$

fieldN $(4$, numfaN $)=$ 'BETA ANALYSES'

C

fdlenN $(4$, numfdN $)=13$

numfdN=numfdN +1

fieldN $(1$, numfdN $)=$ 'R. P. WELLS (RICH)'

falenN $(1$, numfdN $)=18$

fieldN $(2$, numfaN $)={ }^{\prime} 6-7870^{\prime}$

falenN $(2$, numfdN $)=6$

fieldN (3, numfdN) ='RADIOCHEMISTRY, MS 7111; TRA-661, ROOM 129/130'

fdlenN $(3$, numfdN) $=46$

fieldN $(4$, numfdN $)=$ 'BETA ANALYSES'

C

fdlenN $(4$, numfdN $)=13$

do $i=1$, numfdN

farown $(i)=i+1$

fdcoln $(i)=2$

$f i \operatorname{colN}(i)=25$

enddo 


\section{F. SCREEN TEMPLATES SUBMITTED TO BUILD_SCREEN}

\section{F.1 Screen Template for the Main (M) Screen}

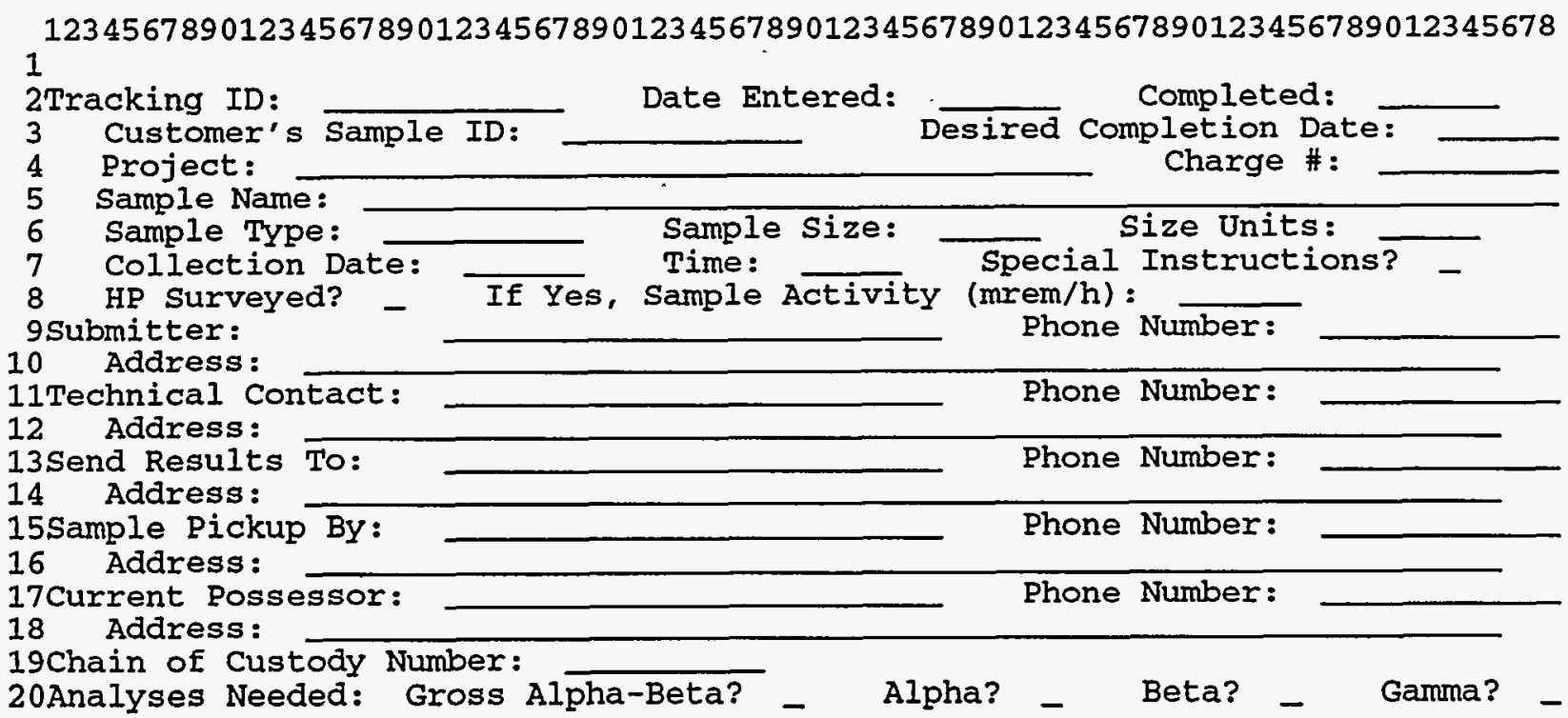

\section{F.2 Screen Template for the Possessors ( $T$ for tracking) Screen}

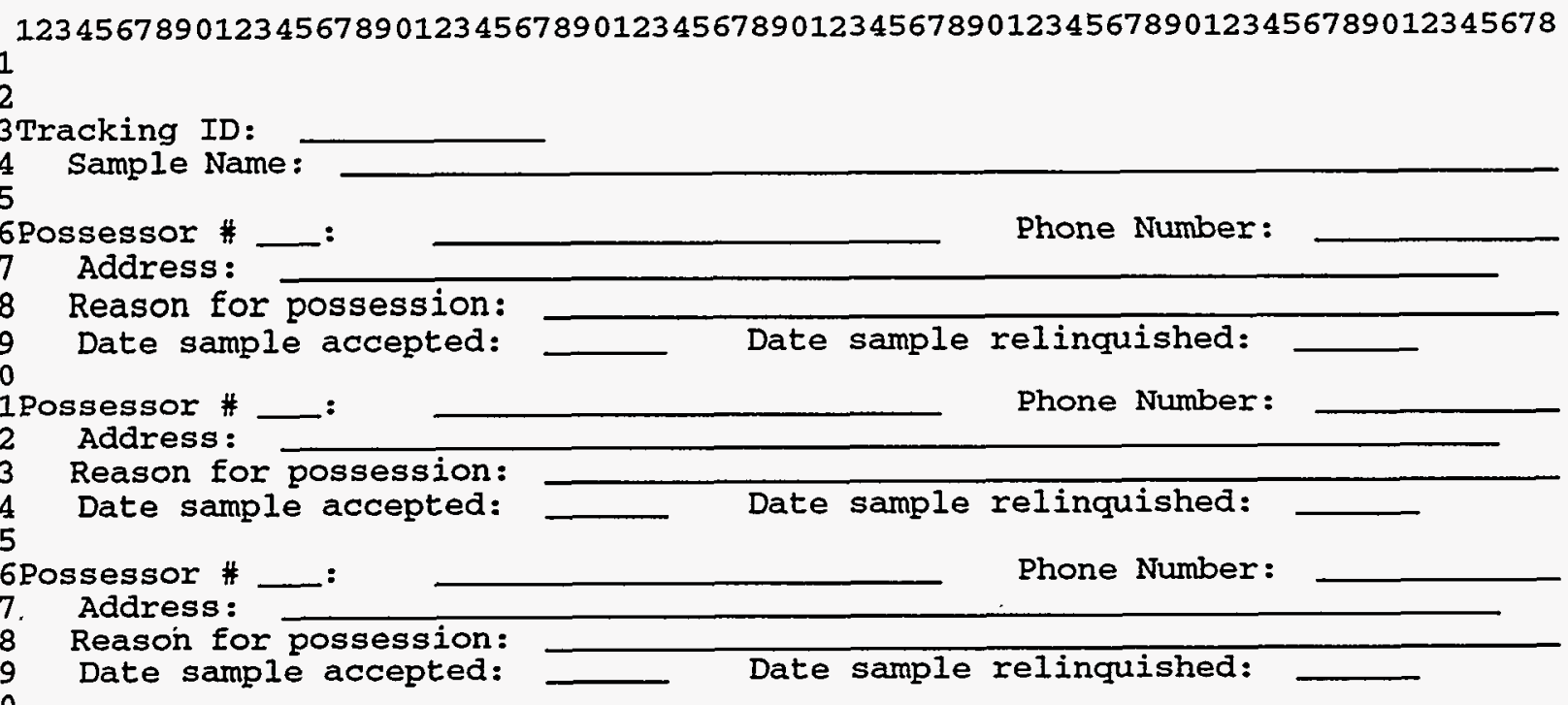




\section{F.3 Screen Template for the Alpha Analyses (A) Screen}

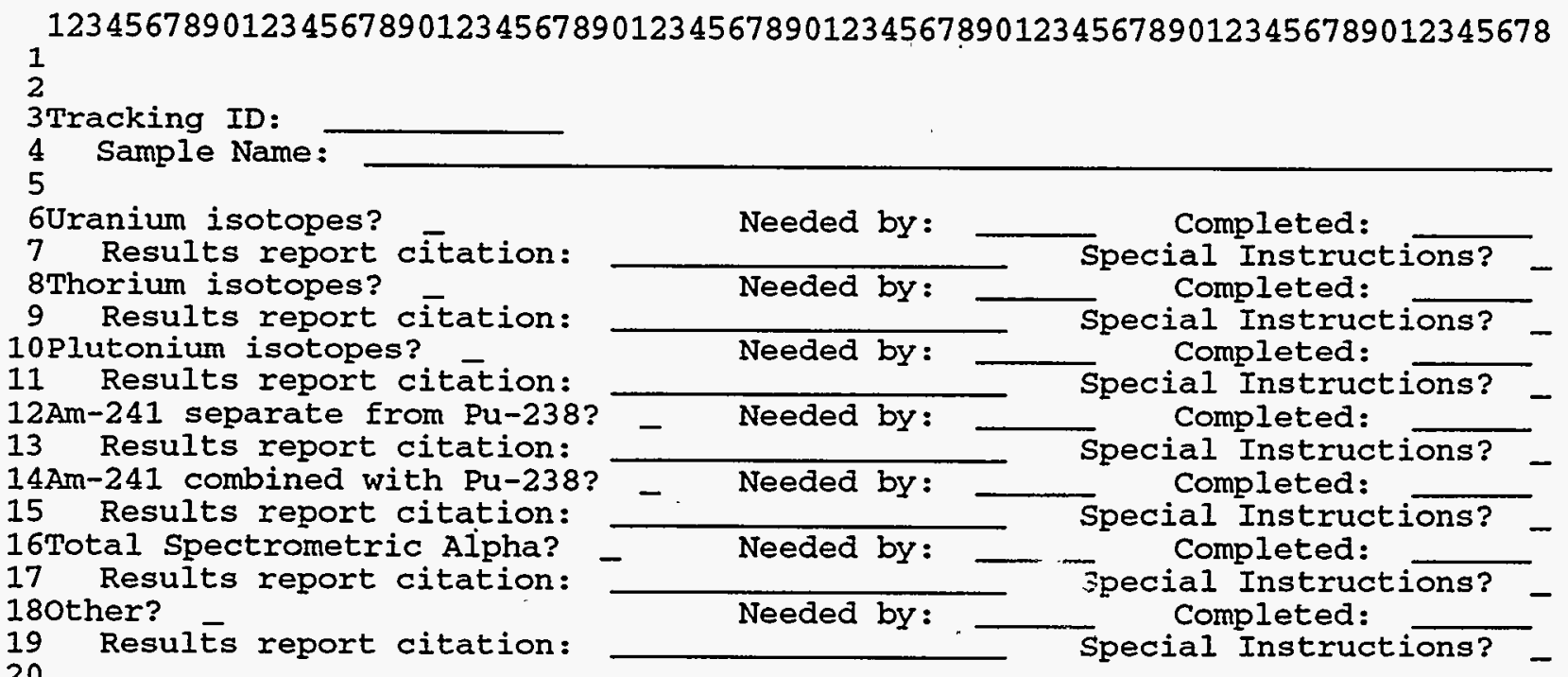

\section{F.4 Screen Template for the Beta Analyses (B) Screen}

2Tracking ID: Sample Name

4 strontium-90?

6 Results needed by: date:

7 Results report citation:

8Strontium-39 and -90 ?

9 Results needed by: date:

10 Results report citation:

11Total strontium?

12 Results needed by: date:

13 Results report citation:

14Tritium?

15 Results needed by: date:

16 Results report citation:

170ther?

18 Nickei-63? Iron-55?

19 Results needed by: date:

20 Results report citation:

Length of count:

time:

Length of count:

time:

Length of count:

time:

Length of count:

time:

Length of count: Sulfur -35 ? time: min or hr?

Completed:-

Special Instructions? min or hr?

Completed:-

Special Instructions? min or hr?

Completed:

Special Instruct $\overline{i o n s ?}$ min or hr?

Completed:

Special Instructions? min or hr?

Plutonium-241? -

Completed: Special Instructions? 


\section{F.5 Screen Template for the Gross Alpha-Beta Analyses (C for combined) Screen}

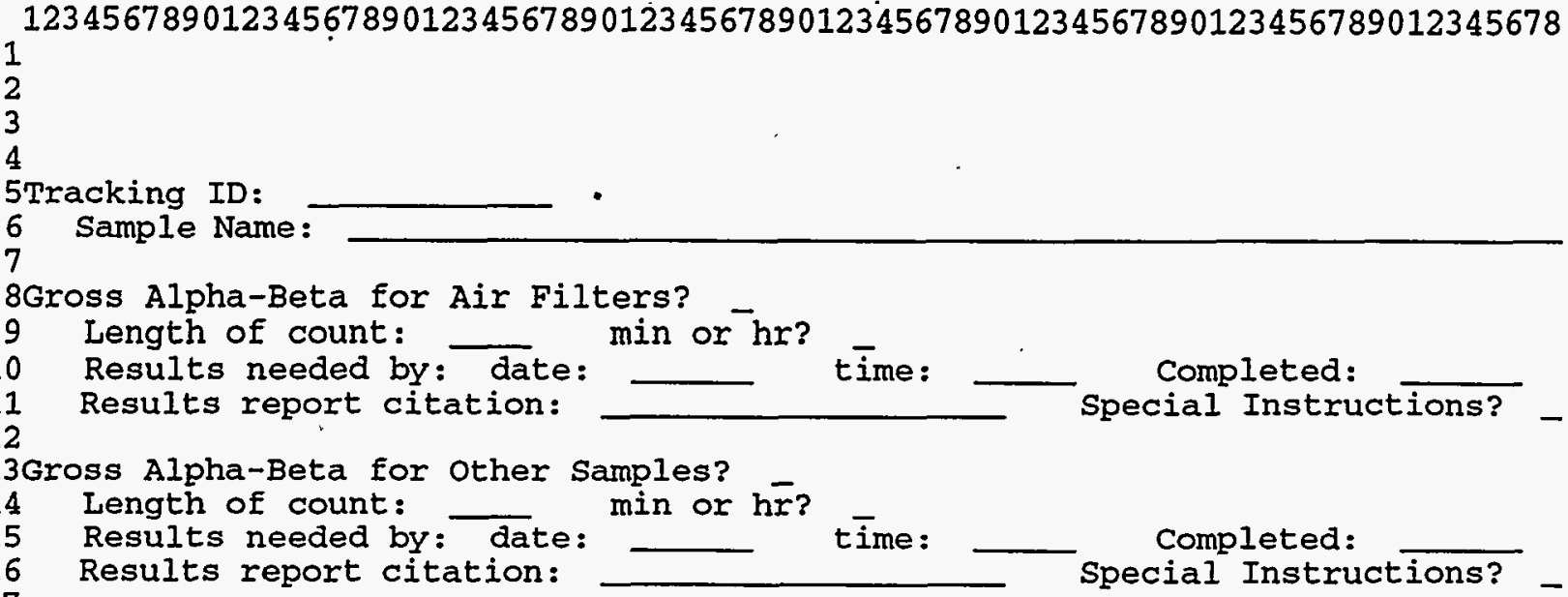

\section{F.6 Screen Template for Gamma Analyses (G) Screen}

123456789012345678901234567890123456789012345678901234567890123456789012345678

1

3Tracking ID:

4 Sample Name:

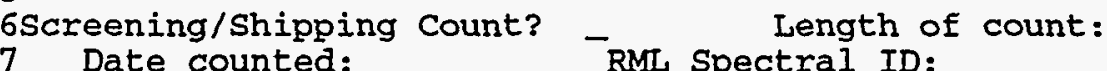

Date counted: RML Spectral ID:

Results needed by: date:

time:

min or hr? -

Results report citation:

Recount? -

10 .

11Full Isotopic Gamma Analysis? - Length of count:

12 Date counted: RML Spectral ID:

13 Results needed by: date

14 Results report citation:

15

160ther Gamma Analysis? -

Date counted:

time:

Completed:

189012345678

17

18

Results needed by: date:

Length of count:

RMI Spectral ID:

Results report citation:

time:

Completed : 


\section{F.7 Screen Template for Special Instructions (I) Screen}

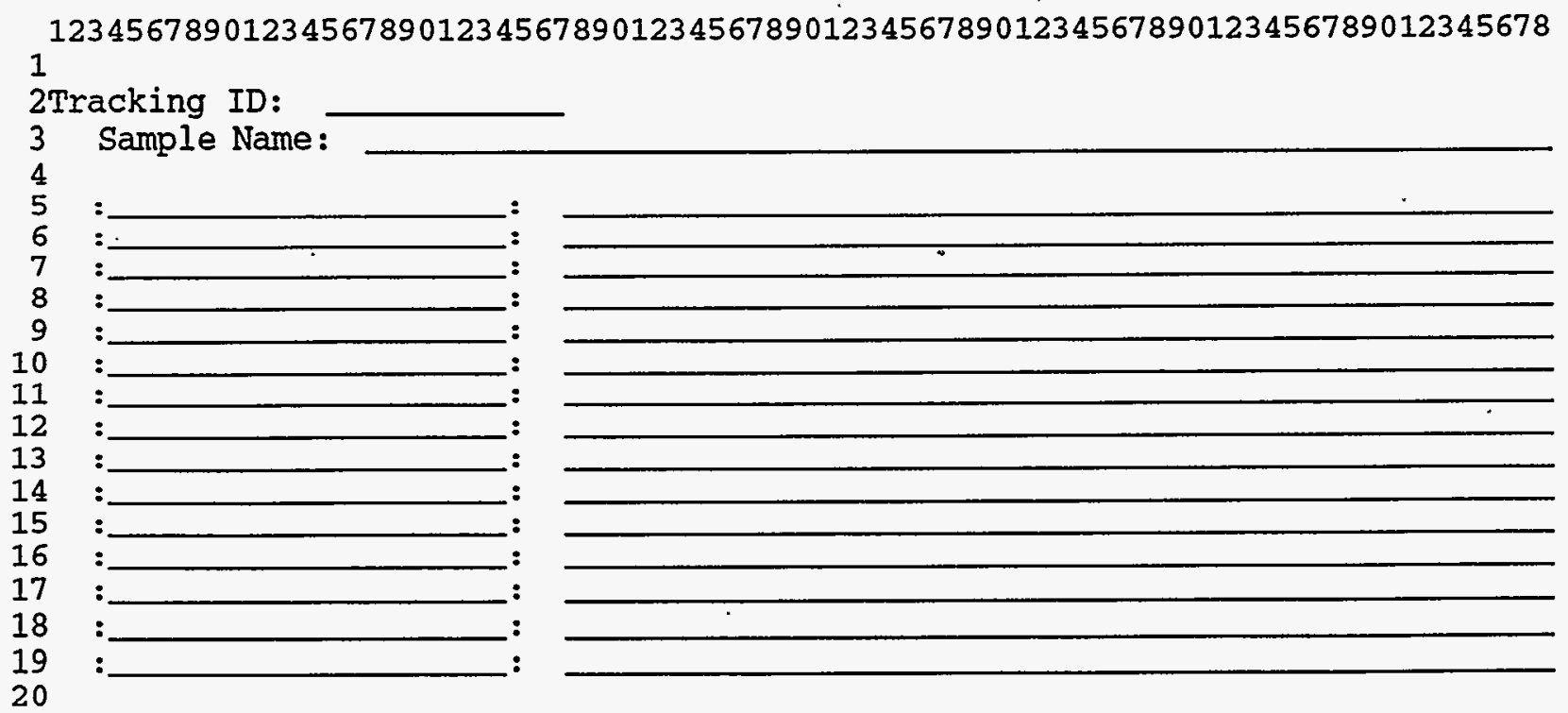

\section{F.8 Screen Template for RML/Analytical Radiochemistry Analysts ( $N$ for names) Screen}

123456789012345678901234567890123456789012345678901234567890123456789012345678 1

2 J. L. Doherty (Jodie) -

3 T. J. Haney (Tom) -

4 R. K. Murray (Ron) -

5 C. L. Rowsell (Cal) -

6 C. W. Sill (Claude) -

7 D. S. Sill (Dave) -

8 L. D. Smith (Larry) -

9 G. K. Taylor (Gene) -

10 L. A. Weinrich (Lou) -

11 R. P. Wells (Rich) -

12 Other

13

14

15

16

17

18

19

20 